\title{
Applying Stable Carbon Isotopic Analysis at the Natural Abundance Level to Determine the Origin of Docosahexaenoic Acid in the Brain of the Fat-1 Mouse
}

By

Vanessa Giuliano

A thesis submitted in conformity with the requirements

for the degree of Master of Science

Department of Nutritional Sciences

Faculty of Medicine

University of Toronto

(C) Copyright by Vanessa Giuliano, 2018 


\title{
Applying Stable Carbon Isotopic Analysis at the Natural Abundance Level to Determine the Origin of Docosahexaenoic Acid in the Brain of the Fat-1 Mouse
}

\author{
Vanessa Giuliano \\ Master of Science \\ Department of Nutritional Sciences \\ Faculty of Medicine \\ University of Toronto
}

2018

\begin{abstract}
Current methods applied to study the origin and metabolism of brain fatty acids involve the administration of synthesized fatty acid tracers. The high cost of these tracers makes it infeasible to conduct chronic studies through this approach, limiting study durations to acute time points that may not reflect the chronic, steady-state, metabolism of fatty acids. Here, we provide the proof-of-concept of a cost-efficient alternative method: natural abundance compound specific isotope analysis by isotope ratio mass spectrometry. Through the application of this method, we differentiated between tissue docosahexaenoic acid (DHA) originating directly from diet or DHA biochemically synthesized in a complex transgenic model: the fat-1 mouse. We further determined pre-formed dietary DHA to be the main source of brain DHA in fat-1 mice fed a diet rich in n-6 and/or n-3 PUFA. Future studies could utilize this approach to answer complex question pertaining to brain fatty acids origin and metabolism.
\end{abstract}




\section{Acknowledgements}

There are many individuals who contributed to the successful completion of this thesis.

Firstly, I would like to extend immense gratitude to my supervisor and mentor, Dr. Richard P. Bazinet. It was an absolute joy to be a part of his team throughout my undergraduate and graduate studies. His kindness fostered a work environment that made me excited to come into work and his critical analysis of scientific literature allowed me to walk away from each conversation we've had with a unique perspective. I will never forget the incredible support of my career goals that he showed me. It is because of him and his mentorship that I am able to fulfill my dreams of entering into the field of medicine.

My Bazinet lab family has provided me with endless support throughout this experience. I cannot thank Dr. Marc-Olivier Trépanier and Dr. Kathryn Hopperton enough for their early mentorship as I embarked on my first research projects in my undergraduate studies and for the encouragement they provided me to pursue a graduate degree. Their advice, support, and friendship have meant the world to me. R.J Scott Lacombe provided valuable guidance and expertise throughout my research project. His patience and assistance when helping me formulate my data and his daily humour have always been appreciated. Finally, I must extend thanks to Dr. Adam Metherel, Dr. Raphaël Chouinard-Watkins, Dr. Lin Lin and Shoug Alashmali who's guidance, kind-heartedness and friendship have made my time at the Bazinet lab incredibly enjoyable!

Many individuals within the Department of Nutritional Sciences at the University of Toronto have also positively impacted this experience. My advisory committee, Dr. Elena Comelli and Dr. Harvey Anderson, provided me with valuable insight that guided my project. Their mentorship, encouragement, and thoughtful comments allowed me to look at my data from a unique perspective and become a better scientist. Dr. Anthony Hanley served as my thesis defense examiner. His insightful comments and discussion were very much appreciated. Our administrative staff, Louisa Kung and Emiliana D'Souza were incredibly helpful throughout this experience and aided me in staying organized. I also feel very fortunate to have shared my 
experience at the University of Toronto with Dr. Ruslan Kubant who's constant encouragement and conversation always brightened my day.

I am very grateful to have received valuable technical assistance throughout my project. Nancy Thomas and the staff of the University of Toronto Department of Comparative Medicine assisted with the care of our mouse colony and provided very useful advice concerning the well- being of our animals. Ashley St. Pierre at the Analytical Facility for Bioactive Molecules of the Centre for the Study of Complex Childhood Diseases at the Hospital for Sick Children assisted with the isotope-ratio mass spectrometry analysis and provided expertise that aided me in further understanding this tool. Without the assistance of these individuals, the completion of my project would not have been possible.

To my closest friends, Dana Goschy, Vanessa Palladini, Sabrina Gava (Guava), and Carl De Guzman, I cannot thank them enough for listening to countless hours about theories they did not understand, providing me with laughter each and every day of my life and for always being ready to pick me up with a coffee in hand whenever I needed a break. Their kindness and generosity over the last twenty-one years of my life cannot be repaid!

Finally, my family has provided me with endless support throughout this experience. My Nonni, Concetta and Antonio Santangelo and Mariangela and Antonio Giuliano, have shown me what hard work and determination looks like. I cannot thank them enough for the endless food, care and love they've provided me throughout my education. My parents, Sam and Grace Giuliano and my siblings, Marieangela and Anthony Giuliano and my brother-in-law Francesco Angeloni have always supported and encouraged my education, even when they didn't always quite understand what I was doing. My mother has been my biggest cheerleader throughout my life and education. She has worked and sacrificed in ways unimaginable to afford me every opportunity possible. I would not be even close to where I am today without her. It is to her I dedicate this thesis. 


\section{Table of Contents}

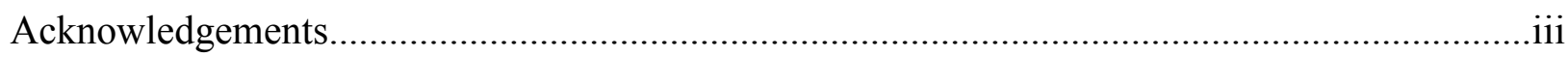

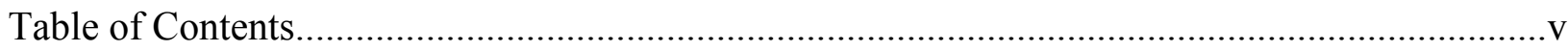

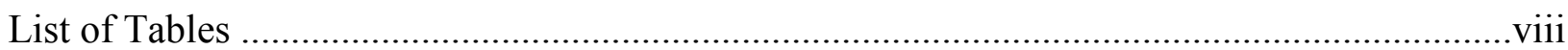

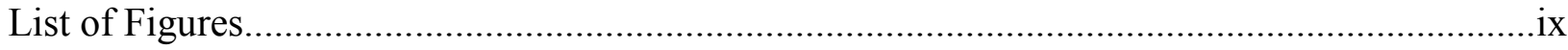

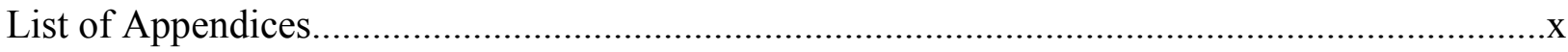

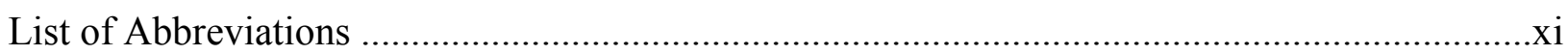

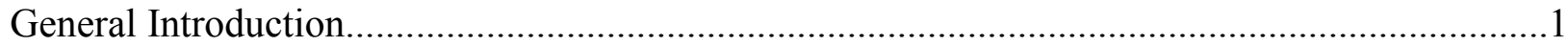

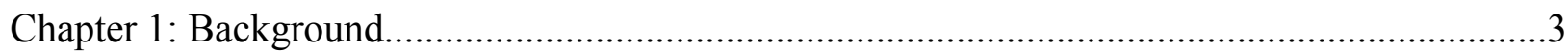

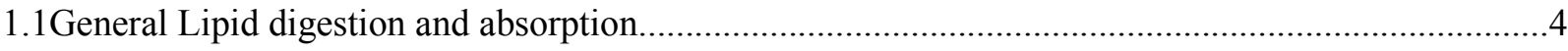

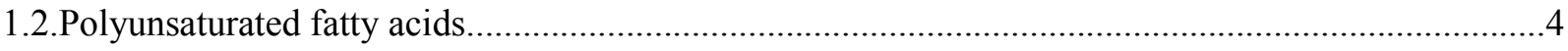

1.3. Sources and synthesis of PUFA............................................................................................

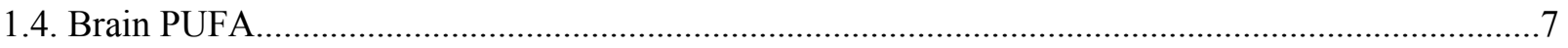

1.5. Brain PUFA uptake ……………………………………………………………………...

1.6. Methods commonly utilized to assess PUFA origin and metabolism in animals................................9

1.7 Natural abundance compound specific isotope analysis by isotope ratio mass spectrometry............10

1.7.1 What is an isotope? ..................................................................................................10

1.7.2 Carbon isotopic fractionation...................................................................................12

1.7.3 Reporting of stable carbon isotope ratios: the delta notation.................................................12

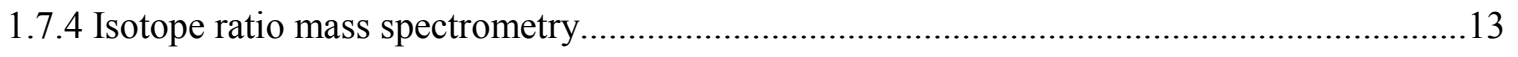

1.7.5 Carbon isotopic fractionation in the food supply............................................................13

1.7.6 Natural abundance compound specific isotope analysis.....................................................17

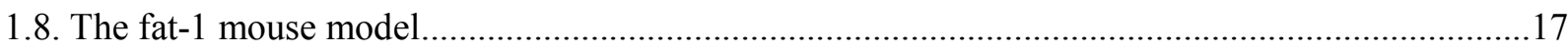

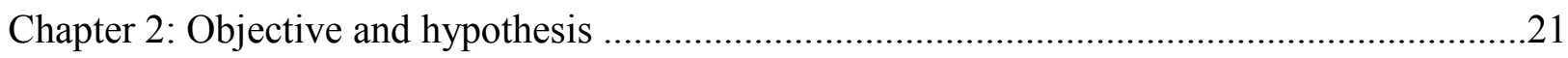


Chapter 3: Applying stable carbon isotopic analysis at the natural abundance level to determine the origin of docosahexaenoic acid in the brain of the fat-1 mouse. .23

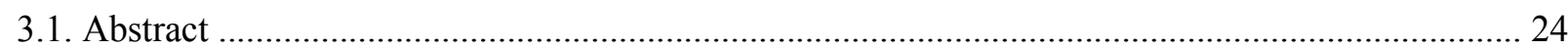

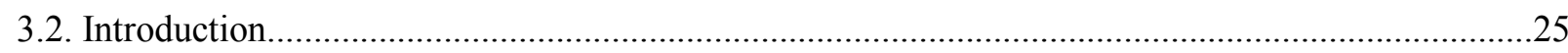

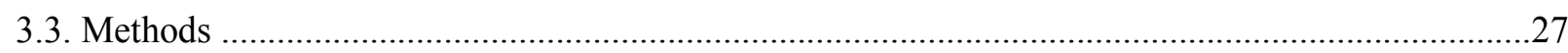

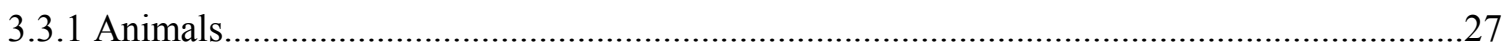

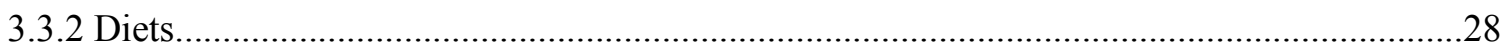

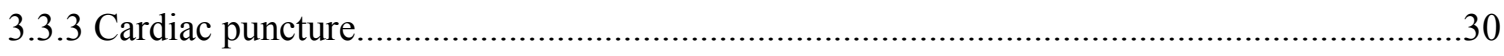

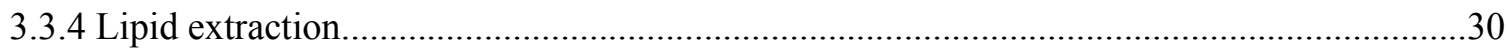

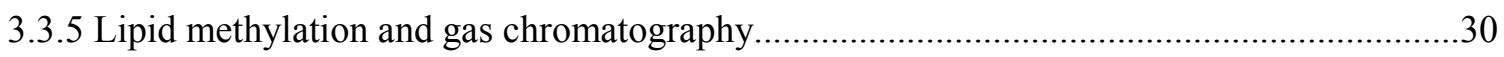

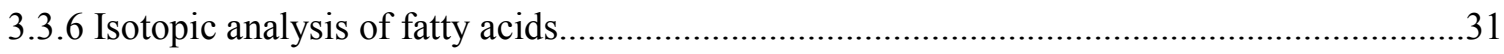

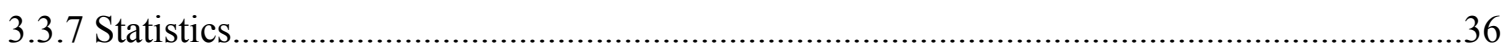

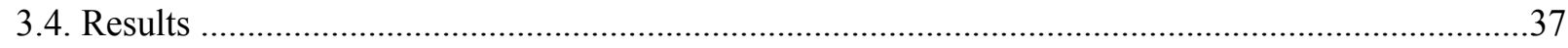

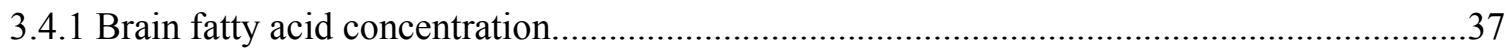

3.4.2 CSIA by IRMS determines the origin of brain DHA.........................................................

3.4.3 Addition of fish oil to the n-6 PUFA rich diets may down regulate synthesis of n-3 PUFA: mechanistic evidence provided by CSIA by IRMS..................................................................

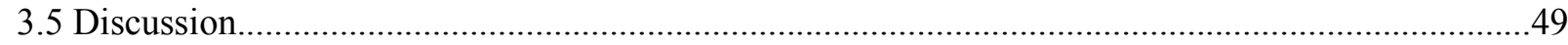

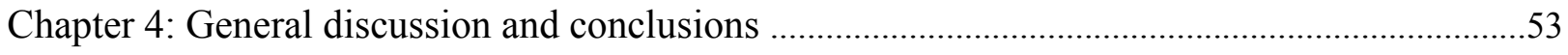

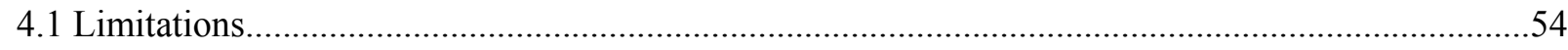

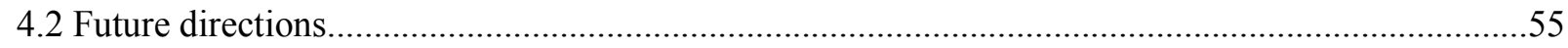

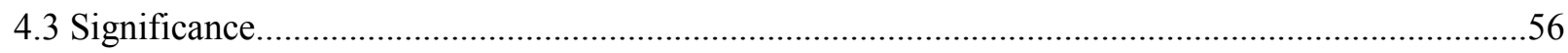

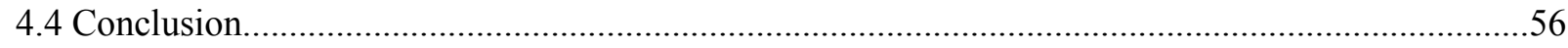

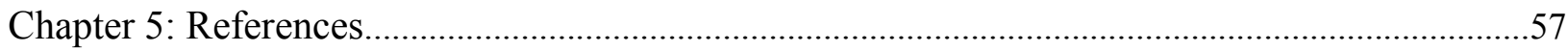


Chapter 6: Appendix 1: Confirmation of dietary fatty acid source via natural abundance

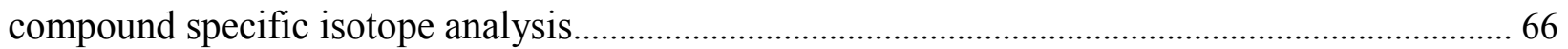

Chapter 7: Appendix 2: Whole brain fatty acid composition ........................................................69 


\section{List of Tables}

Table 1.7.1.1 Global natural isotopic abundance of commonly studied stable isotopes...............9

Table 3.3.2.1 Fatty acid composition and $\delta^{13} \mathrm{C}$ values of key dietary fatty acids......................27

Table $6.1 \delta^{13} \mathrm{C}$ values of key fatty acids in experimental diets.........................................66

Table 7.1 Brain whole brain fatty acid concentrations of wild-type and fat- 1 mice fed the $10 \%$

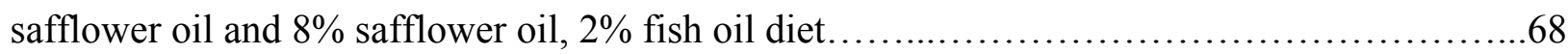

Table 7.2 Brain whole brain fatty acid concentrations of wild-type and fat- 1 mice fed the $10 \%$

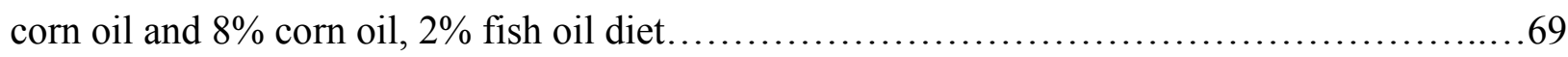




\section{List of Figures}

Figure 1.3.1 n-3 PUFA and n-6 PUFA synthesis pathway..............................................

Figure 1.7.5.1 The different modes of photosynthesis of plants within the food supply..............14 Figure 1.8.1 Enzymatic conversion of n-6 PUFA to n-3 PUFA via the n-3 desaturase enzyme in fat-1 mice. 17

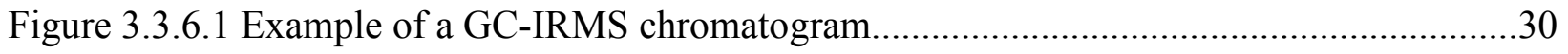

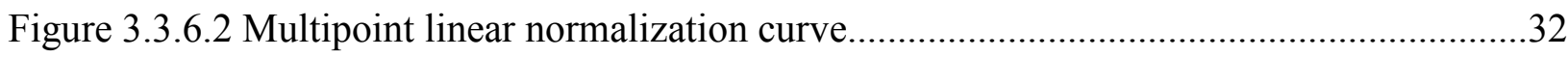

Figure 3.4.1.1 Brain DHA and ARA concentrations and $\delta^{13} \mathrm{C}$ values of animals fed either the $10 \%$ safflower oil diet or $8 \%$ safflower oil, $2 \%$ fish oil diet.

Figure 3.4.1.2 Brain DHA concentrations and $\delta^{13} \mathrm{C}$ values of animals fed either the $10 \%$

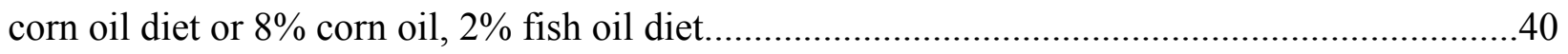

Figure 3.4.2.1 Fat-1 mouse conversion of n-6 PUFA to n-3 PUFA.........................................41

Figure 3.4.2.2 Study design overview and depiction of the hypothetical routes by which fat-1 mice may obtain brain DHA. .42

Figure 3.4.3.1 Liver DHA concentrations and $\delta^{13} \mathrm{C}$ values of animals fed either the $10 \%$ safflower oil diet or $8 \%$ safflower oil, $2 \%$ fish oil diet.

Figure 3.4.3.2 Liver DHA concentrations and $\delta^{13} \mathrm{C}$ values of animals fed either the $10 \%$ corn oil diet or $8 \%$ corn oil, $2 \%$ fish oil diet. .46 


\section{List of Appendices}

Appendix 1 Confirmation of dietary source via compound specific isotope analysis..........64

Appendix 2 Whole brain fatty acid composition...........................................67 


\title{
Abbreviations
}

\author{
ALA- alpha-linolenic acid \\ ANOVA- analysis of variance \\ APE- atom percent excess \\ ARA- arachidonic acid \\ BBB- blood-brain barrier \\ ${ }^{12} \mathrm{C}-12$ - carbon \\ ${ }^{13} \mathrm{C}-13$ - carbon \\ cPLA 2 - calcium-independent phospholipase $\mathrm{A}_{2-}$ \\ CAM- crassulacean acid metabolism \\ CO-corn oil \\ $\mathrm{CO}_{2}$ - carbon dioxide \\ CSIA- compound specific isotope analysis \\ COX- cyclooxygenase \\ $\mathrm{CrO}_{3}$ - chromium trioxide \\ $\mathrm{Cu}$ - copper \\ 813C- delta ${ }^{13}$ carbon \\ DHA- docosahexaenoic acid \\ DPA- docosapentaenoic acid \\ EA- elemental analysis \\ EPA- eicosapentaenoic acid \\ FAME- fatty acid methyl esters \\ FO- fish oil \\ GC- gas chromatography \\ ${ }^{1} \mathrm{H}$ - 1-hydrogen \\ ${ }^{2} \mathrm{H}$ - 2-hydrogen \\ h- hour \\ HETE- hydroxyeicosatetraenoic acid \\ $\mathrm{H}_{2} \mathrm{O}$-water \\ IRMS- isotope ratio mass spectrometry \\ LA-linoleic acid \\ LDL-low density lipoprotein \\ min- minute \\ $\mathrm{m} / \mathrm{z}$ - mass/charge \\ n- omega \\ ${ }^{14} \mathrm{~N}-14$-nitrogen \\ ${ }^{15} \mathrm{~N}-15$-nitrogen \\ $\mathrm{Ni}$ - nickle \\ ${ }^{16} \mathrm{O}$ - 16-oxygen \\ ${ }^{17} \mathrm{O}$ - 17-oxygen \\ ${ }^{18} \mathrm{O}$ - 18-oxygen \\ Phosphoenolpyruvate carboxylase- PEP C \\ PG- prostaglandin \\ $\mathrm{PGI}_{2}$ - prostacyclin \\ $\mathrm{PLA}_{2}$ - phospholipase $\mathrm{A}_{2}$
}


PUFA- polyunsaturated fatty acid or acids

Ribulose 1,5-bisphosphate- RuBP

Ribulose bisphosphate carboxylase- Rubisco

${ }^{32}$ S- 32-sulfur

${ }^{33} \mathrm{~S}-33$-sulfur

${ }^{34} \mathrm{~S}-34$-sulfur

s- second

SD- standard deviation

SIM- select ion monitoring

SO- safflower oil

TX- thromboxane

Urey- mUr

VLDL- very low density lipoprotein

VPDB- vienna Pee Dee Belemnite

WT- wild type 


\section{General Introduction}

DHA is the most abundant n-3 PUFA in the brain and is a fatty acid that humans cannot synthesize de novo and therefore must consume directly from the diet. DHA is known to play a crucial role in the maturation of visual acuity and neurodevelopment. Further, a host of studies have suggested that low intakes of n-3 PUFA may be associated with certain neurological and psychiatric disorders while metabolites of n-3 PUFA have also been suggested to play a role in the resolution of neuroinflammation. As a result of this physiological importance, it is crucial to understand the origin and metabolism of brain DHA. However, methodological limitations exist when attempting to understand the chronic metabolism of tissue fatty acids. Currently, common methods employed to study the origin of tissue n-3 PUFA involve the administration of expensive fatty acid tracers. The large cost associated with the use of these tracers limits study durations to acute time points which may not reflect the chronic metabolism and turnover of tissue fatty acids.

The objective of this thesis was to provide the proof-of-concept of a novel, cost-efficient approach to study the origin of brain fatty acids: natural abundance compound specific isotope analysis by isotope ratio mass spectrometry (CSIA by IRMS). We did so through applying this method to answer a question that was previously infeasible to answer: the main origin of brain DHA in fat-1 mice.

In nature, distinct plant types exhibit unique carbon isotope ratios characteristic of their environment and preferred carbon fixation pathway. As a result, when fatty acids derived from plants are consumed, their incorporation into animal tissues can be traced via their unique carbon 
isotopic "fingerprint" through the use of gas-chromatography-IRMS. Thus, by feeding animals diets that are naturally isotopically distinguished, this technique can allow for the chronic tracing of fatty acids in vivo while evading the need to purchase expensive fatty acid tracers.

In the present study, we apply CSIA by IRMS to determine the origin of brain DHA in fat-1 mice. When fed a diet rich in n-3 and n-6 PUFA, it was unknown if fat- 1 mice obtain their brain DHA directly from dietary n-3 PUFA, or through the synthesis of n-3 PUFA from dietary n-6 PUFA. Through isotopically analyzing the brains of fat- 1 mice fed diets in which the main n-3 and n-6 PUFA were naturally isotopically distinguished, we identified dietary n-3 PUFA as the main origin of DHA in fat-1 mice. We subsequently isotopically analyzed liver tissue of these animals to understand the mechanism by which this source may be preferred. Isotopic analysis of liver tissue provided evidence that when fed a diet rich in n-3 and n-6 PUFA, hepatic synthesis of n-3 PUFA from n-6 PUFA sources may be downregulated. 
Chapter 1:

Background 


\subsection{General lipid digestion and absorption}

Lipid digestion commences in the mouth, where lingual lipase enzymatically cleaves fatty acids from the sn-3 position of triacylglycerols. Upon leaving the oral cavity, lipid enzymatic digestion continues in the stomach by gastric lipase which continues to cleave fatty acids from triacylglycerols at the sn-3 position. Lipids then move to the small intestine where their presence stimulates the secretion of cholecystokinin (CCK). CCK results in the release of bile which aids in the emulsification of lipids. This emulsification enhances the surface area of fatty acids available to interact with pancreatic lipase which hydrolyzes fatty acids at the sn-1 and sn-3 positions of triacylglycerols. Free fatty acids and 2-monoacylglycerol digestion products are then absorbed into the bloodstream or lymphatic system. Short and medium chain fatty acids can be transported into the bloodstream through the hepatic portal vein system, bound to albumin (a liver synthesized carrier protein). Polyunsaturated fatty acids (PUFA) have been proposed to be passively absorbed into enterocytes via flip-flop mechanisms or through facilitated transport using fatty acid translocase (CD) 36 and fatty acid transport proteins (FATP). Once within the enterocyte fatty acids are acted upon by acyl-CoA synthetase which catalyzes the addition of a CoA molecule onto fatty acid acyl chains. Fatty acid-CoA molecules then bind to fatty acid binding proteins and are directed to the smooth endoplasmic reticulum where complex lipids are resynthesized and packaged into chylomicrons. Chylomicrons will be then released into the lymphatic system and eventually enter the bloodstream via the thoracic duct. Fatty acids esterified within chylomicrons are subsequently released via the action of lipoprotein lipase or endothelial lipase for uptake into bodily tissue, or can be re-esterified to a glycerol backbone for storage as triacylglycerols in adipose tissue. Chylomicron remnants and circulating free fatty acids eventually enter the liver where they can be beta-oxidized, used as precursors for the synthesis of longer chain fatty acids, or packaged into very low density lipoproteins and released from the liver for lipid delivery to tissue[1,2].

\subsection{Polyunsaturated fatty acids}

PUFA are fatty acids (hydrocarbon chains with a terminal carboxyl group) with more than one double bond. There are three main types of PUFA, omega(n)-3 PUFA, n-6 PUFA and n-9 PUFA. The first double bond possessed by these fatty acids occurs at the third, sixth and ninth carbon from the methyl end of the acyl chain, respectively. PUFA can be found in free form or esterified to phospholipids, lysophospholipids, cholesteryl esters, and as triacylglycerols[1]. N-3 and n-6 
PUFA have been deemed nutritionally essential as humans cannot synthesize them de novo. As n-9 PUFA are not assessed in any analysis described in this thesis, they will not be mentioned further.

\subsection{Sources and synthesis of PUFA}

As mentioned, humans cannot synthesize n-3 and n-6 PUFA de novo and they, therefore, must be obtained by dietary means. Long chain PUFA such as eicosapentaenoic acid (20:5n-3, EPA) and docosahexaenoic acid (22:6n-3, DHA) are commonly consumed from marine sources such as fatty fish[3]. EPA and DHA can also be synthesized, predominately in the liver, from alphalinolenic acid (18:3n-3, ALA), their fatty acid precursor (Figure 1.3.1). ALA is typically consumed in foods such as nuts, plant seeds and oils derived from these sources (ie. soybean oil, canola oil, and flaxseed oil). The conversion of ALA to DHA is accomplished by a series of desaturation and elongation enzymatic reactions. However, this conversion is thought to be rather inefficient, with the synthesis rate of ALA to DHA estimated to be $\sim 1 \%[4,5]$. Despite this, DHA synthesis from ALA may still be sufficient to supply the brain[6].

N-6 PUFA such as arachidonic acid (20:4n-6, ARA) are consumed in meat and meat products, particularly those derived from animals fed a corn-based diet[7]. As with n-3 PUFA, longer chain n-6 PUFA can be synthesized from n-6 PUFA precursors such as linoleic acid (18:2n-6, LA). LA is found in plant oils such as safflower oil and corn oil. The n-6 PUFA synthesis pathway utilizes the same enzymes used for n-3 PUFA synthesis. N-3 and n-6 PUFA precursors, therefore, compete for enzyme availability. As a result, shifting the ratio of dietary n-6 to n-3 PUFA can alter synthesis levels[8,9]. 
n-3 PUFA synthesis pathway

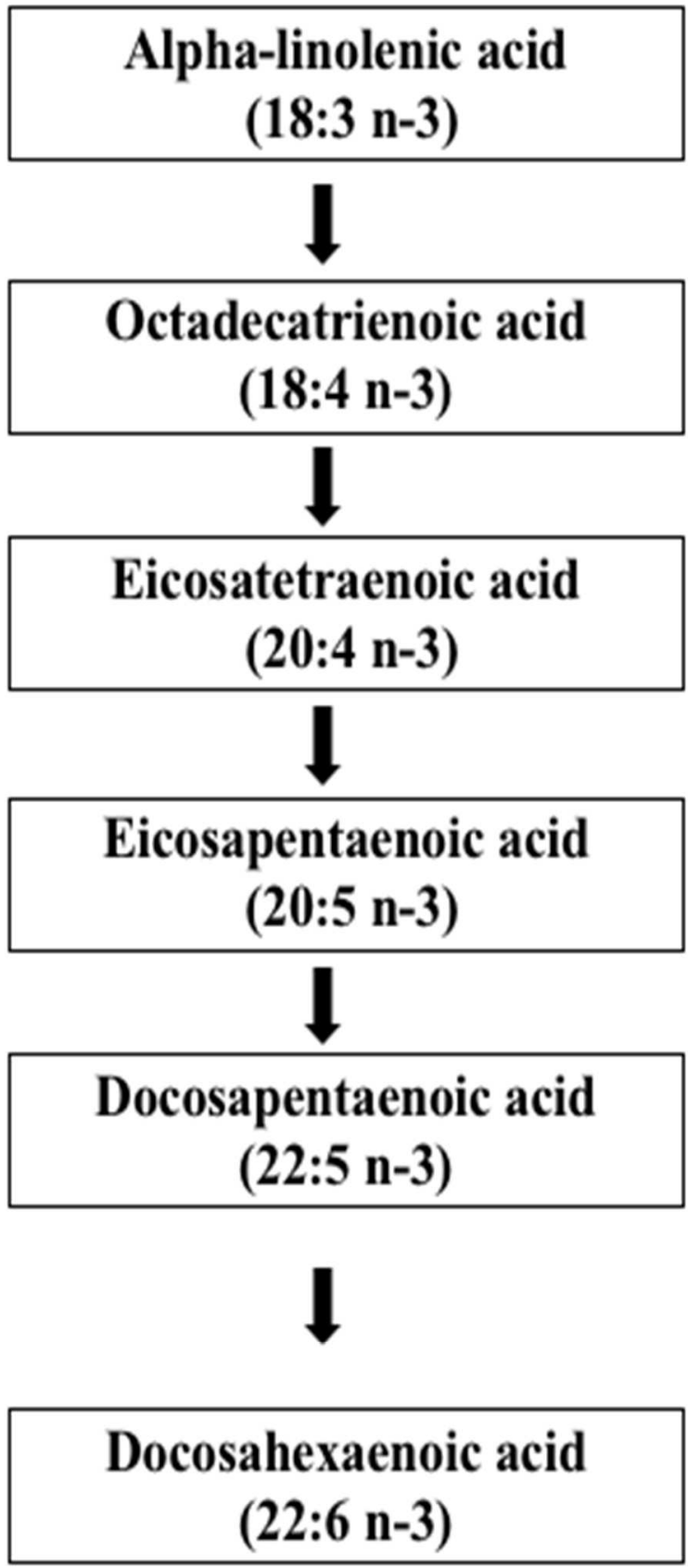

n-6 PUFA synthesis pathway

Linoleic acid

(18:2 n-6)

$\Delta$-6-desaturase

Elongase

Gamma-linolenic acid (18:3 n-6)

$\Delta$-5-desaturase

Dihomo-gamma-linolenic acid (20:3 n-6)

Arachidonic acid (20:4 n-6)

Elongase Elongase $\Delta$-6-desaturase $\beta$-oxidation
Adrenic acid

(22:4 n-6)
Docosapentaenoic acid (22:5 n-6)

Figure 1.3.1 n-3 PUFA and n-6 PUFA synthesis pathway 


\subsection{Brain PUFA}

PUFA make up approximately $35 \%$ of brain lipids, with the most abundant PUFA species being docosahexaenoic acid (22:3n-3) and arachidonic acid (20:4n-6). PUFA are thought to play crucial physiological roles within the brain. DHA and ARA are highly enriched within neuronal membranes and are important for neurotransmission, neuroprotection, and neurogenesis[10-12]. Both DHA and ARA are crucial for proper neurodevelopment while DHA plays an essential role in the maturation of visual acuity[13-15]. Low intake of n-3 PUFA have been linked to increased neurological and psychiatric disorders [16,17]. Further, an imbalance in the ratio of n-3 to n-6 PUFA in the blood has also been noted in several mood disorders[18-20] and in individuals experiencing cognitive decline[21].

Through the action of calcium-independent phospholipase $\mathrm{A}_{2}\left(\mathrm{PLA}_{2}\right)$ and calcium-dependent $\mathrm{PLA}_{2}$, respectively, non-esterified DHA and ARA can be released from phospholipids and be metabolized into bioactive lipid-derived mediators. ARA bioactive mediators are known as eicosanoids. When acted upon by the enzyme cyclooxygenase (COX) 1 and 2 ARA can be broken down into prostaglandins (ie. $\mathrm{PGD}_{2}, \mathrm{PGE}_{2}, \mathrm{PGF}_{2}, \mathrm{PGI}_{2}$ ) and thromboxanes (ie. $\mathrm{TXA}_{2}$ and $\mathrm{THB}_{2}$ ). ARA can also be acted upon by lipoxygenase (LO) enzymes (5-LO, 12-LO, and 15LO) to produce hydroxyeicosatetraenoic acid (HETE) molecules and subsequently lipoxins and leukotrienes or be acted upon by cytochrome p450 to create epoxides and HETE molecules. ARA mediators are thought to be generally pro-inflammatory and play a role in the regulation of pain, blood flow and thrombosis[22,23].

In contrast, DHA derived bioactive mediators are known as docosanoids. Non esterified DHA can either be acted upon by 12-LO or 15-LO to produce resolvins, protectins and maresins. These mediators have also been suggested to play a key biological role in neuroprotection, the resolution of inflammation, and protection against oxidative stress[24].

\subsection{Brain PUFA uptake}

It is understood that minimal production of PUFA occurs within the brain. It is therefore accepted that the brain must uptake circulating PUFA[25,26]. The mechanism by which this occurs has been a topic of debate. PUFA esterified in lipoproteins, plasma non-esterified PUFA, 
and lysophospholipids have all been proposed as available pools for brain PUFA uptake. Although lipoproteins do not transverse the blood-brain-barrier (BBB), low-density lipoprotein (LDL) receptors are expressed on the endothelium of the $\mathrm{BBB}[27]$. As a result, it was hypothesized that interactions of lipoproteins with lipoprotein receptors could facilitate the uptake of lipoproteins and PUFA release into the brain[28]. However, LDL receptor and very low LDL (VLDL) receptor knock-out models do not display altered brain PUFA concentrations, suggesting that these receptors are not necessary for brain PUFA uptake[29,30]. It has also been posited that brain PUFA uptake is facilitated through the CD36 transporter, however, abolition of CD36 in mice does not result in decreased brain PUFA concentrations and is therefore nonessential for brain PUFA uptake[31].

DHA contained in the lysophosphatidylcholine class of lipids has also been suggested as the major source of brain DHA[32]. Indeed, in vitro evidence does suggest that lysophosphatidylcholine-DHA is taken up into brain capillary endothelial cells more readily than non-esterified DHA[33]. Further, the brains of 20-day old rat pups intravenously injected with radiolabeled lysophosphatidylcholine-DHA reveal 10 fold higher recovery of radioactivity in the brain than when injected with radiolabeled non-esterified DHA[34]. It is crucial to note, however, that this particular study did not correct for differences in lysophosphatidylcholineDHA and non-esterified DHA half-lives or pool sizes. Also, when conducting these analyses for longer time frames, the administered radiolabeled tracers circulate to the liver where they can be metabolized and repackaged. This can result in radioactivity being distributed to multiple lipid pools which can then become available to the brain for PUFA uptake[35]. To address these potential confounds, Chen et al (2015) assessed brain uptake of radiolabeled non-esterified DHA and lysophosphatidylcholine-DHA by applying short duration steady-state infusion methods while also correcting for differences in pool size and half-life. By this approach, it was determined that the rate of non-esterified DHA brain uptake was 10 fold higher than that of lysophosphatidylcholine-DHA, making it clear that plasma non-esterified DHA is more readily taken up by the brain and likely to be the major lipid pool supplying the brain[35]. Competitive assays have further supported this theory, providing evidence that DHA enters the brain in a noncompetitive manner[36]. This suggests that brain PUFA is derived via passive diffusion of free fatty acids and refutes the possibility of receptor usage. 


\subsection{Methods commonly utilized to assess PUFA origin and metabolism in animals}

Typically, the study of the biological fate of a fatty acid involves the administration of stable $\left({ }^{13} \mathrm{C}\right.$ or $\left.{ }^{2} \mathrm{H}\right)$ or radio $\left({ }^{14} \mathrm{C}\right.$ or $\left.{ }^{3} \mathrm{H}\right)$ labelled fatty acid tracers[35,37-39]. These tracers are then monitored in vivo for their appearance in different tissues, metabolites and blood fractions and can provide valuable information about the metabolism and turnover of fatty acids. Tracers can be administered orally, through gavage feeding, intravenously or directly into the brain through intracerebroventricular infusion. Intravenous administration can involve either bolus injections or steady-state infusion techniques. The appearance of serum tracer metabolites is then assessed after recurrently sampling blood. Steady-state infusion administration is advantageous as it allows for the estimate of whole-body fatty acid synthesis and also can quantify fatty acid synthesis from serum tracer as opposed to post-prandial tracer pools[5,40].

As a result of the high cost of fatty acid tracers (ie. commercial cost of $10 \mathrm{mg}$ of deuterated DHA is $\sim \$ 3000)$, administration is usually limited to single dosages over a relatively short duration (5 min- $3 \mathrm{~h}$ ). This largely limits the data obtained via these approaches. For example, when administered orally, the vast majority of fatty acid tracer that is not beta oxidized may be sequestered in adipose tissue and not available for analysis at acute time points[41]. Further, oral administration also only allows one to investigate the post-prandial biochemical fate of the tracer, which may not be reflective of chronic metabolism or tissue incorporation. Although intravenous administration through steady-state infusion techniques provides the advantage of estimating whole-body fatty acid synthesis, it is still limited to acute time points that may not reflect the long-term metabolism of the fatty acid. Further, the invasive nature of this route of administration may also introduce confounding factors by placing stress on the animal. Ideally, to study the routing and metabolism of fatty acids, all fatty acids of interest in an experimental diet would be replaced with labelled tracers and fed to animals chronically. This approach was attempted once by Lefkowitz et al (2005) in which all dietary n-3 PUFA was replaced with ${ }^{2} \mathrm{H}$ labelled ALA and the fate of this tracer was tracked in vivo upon a 20-day feeding study in rat pups[37]. Synthesizing this custom diet would have likely been remarkably expensive as the commercial cost of deuterated ALA is $\sim \$ 360 / \mathrm{mg}$. This cost makes it clear that this is not a feasible approach to studying fatty acid routing and metabolism and that alternate methods are needed. 


\subsection{Natural abundance compound-specific isotope analysis by isotope ratio mass spectrometry}

\subsubsection{What is an isotope?}

Isotopes are different forms of the same element that have the same number of protons and electrons, but a different number of neutrons. Isotopes containing a higher number of neutrons have a heavier atomic mass than isotopes of the same element with fewer neutrons.

Consequently, all elemental isotopes have similar chemical properties but different physical properties.

Isotopes can exist in either stable or radioactive forms. While radioactive isotopes undergo radioactive decay over time, stable isotopes do not. The most commonly studied naturally occurring stable isotopes are carbon $\left({ }^{12} \mathrm{C},{ }^{13} \mathrm{C}\right)$, nitrogen $\left({ }^{14} \mathrm{~N},{ }^{15} \mathrm{~N}\right)$, sulfur $\left({ }^{32} \mathrm{~S},{ }^{33} \mathrm{~S},{ }^{34} \mathrm{~S}\right)$, oxygen $\left({ }^{16} \mathrm{O},{ }^{17} \mathrm{O},{ }^{18} \mathrm{O}\right)$ and hydrogen $\left({ }^{1} \mathrm{H},{ }^{2} \mathrm{H}\right)$. A large proportion of stable isotopes typically exist in one dominant isotopic form with small but detectable fractions of other isotopic forms (Table 1.7.1.1). Abundances of isotopes are usually reported as global atom percentage. However, a small variation does exist in the abundances of stable isotopes in different earth subsystems. For example, the global abundance of the stable isotope of ${ }^{13} \mathrm{C}$ is reported as $1.11 \%$, however, $75 \%$ of the ${ }^{13} \mathrm{C}$ variation in subsystems usually actually falls between $1.0343-1.1167 \%[42]$. As the focus of this work exploits the natural abundance of carbon isotopes in fatty acids, the remainder of this section will concentrate specifically on carbon isotopes. 


\begin{tabular}{ll}
\hline Elemental isotope & Abundance (atom \%) \\
\hline${ }^{1} \mathrm{H}$ & 99.985 \\
${ }^{2} \mathrm{H}$ & 0.015 \\
${ }^{12} \mathrm{C}$ & 98.89 \\
${ }^{13} \mathrm{C}$ & 1.11 \\
${ }^{14} \mathrm{~N}$ & 99.63 \\
${ }^{14} \mathrm{~N}$ & 0.37 \\
${ }^{16} \mathrm{O}$ & 99.76 \\
${ }^{17} \mathrm{O}$ & 0.04 \\
${ }^{18} \mathrm{O}$ & 0.20 \\
${ }^{32} \mathrm{~S}$ & 95.02 \\
${ }^{33} \mathrm{~S}$ & 0.76 \\
${ }^{34} \mathrm{~S}$ & 4.22 \\
\hline
\end{tabular}

Table 1.7.1.1 Global natural isotopic abundance of commonly studied stable isotopes[42] 


\subsubsection{Carbon isotopic fractionation}

Isotopic fractionation refers to the enrichment of one isotopic form of an element relative to another. There are two main isotope effects that lead to variations in ${ }^{13} \mathrm{C}$ to ${ }^{12} \mathrm{C}$ ratios: kinetic isotopic effects and equilibrium isotopic effects. Kinetic isotope effects relate to the differences in reaction rate when one elemental isotope is substituted for another. Heavier isotopes have a lower kinetic energy and therefore move at a slower rate than lighter isotopes. As a result, heavier isotopes progress through reactions at a slower rate than lighter isotopes and are therefore less favored. This has the greatest effect when the substrate of interest is involved in the rate-limiting step of a reaction. In contrast, equilibrium effects occur as a result of differences in ground state energy. Heavier isotopes create more stable bonds and have a lower ground state energy than lighter isotopes. As a result, heavier isotopes are usually more concentrated in the reactants or product of an equilibrium reaction where it is more strongly bonded. For example, in the dissolution reaction of $\mathrm{CO}_{2},{ }^{13} \mathrm{C}$ is more likely to be found in $\mathrm{HCO}_{3}{ }^{-}$than $\mathrm{CO}_{2}$. Both kinetic and equilibrium isotope effects are most effective at lower temperatures[43,44].

\subsubsection{Reporting of stable carbon isotope ratios: the delta notation}

To allow for inter-laboratory comparison, stable carbon isotopic ratios are expressed relative to an internationally accepted reference standard through the use of the delta notation shown below:

$$
\left.\delta^{13} \mathrm{C}=[(\mathrm{R} \text { sample-R standard }) / \mathrm{R} \text { standard }] \mathrm{x} 1000 \text { (\%o or } \mathrm{mUr}\right),
$$

Where R sample $={ }^{13} \mathrm{C}$ abundance sample $^{12} \mathrm{C}$ abundance sample $_{\text {and }} \mathrm{R}$ standard $={ }^{13} \mathrm{C}$

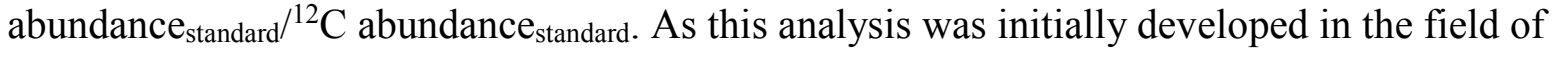
geochemistry, the reference standard initially selected for the analysis of stable carbon isotopes is Pee Dee Belemnite, a marine fossil gathered from the Pee Dee formation in South Carolina. This material is now exhausted from the earth but is still utilized to standardized isotopic measurements and is now referred to as Vienna Pee Dee Belemnite (VPDB). VPDB has a constant $\mathrm{R}$ value of 0.0112372 . As this material is more enriched in ${ }^{13} \mathrm{C}$ than most organic materials, $\delta^{13} \mathrm{C}$ values reported for biological tissues are often negative[45].

As lighter isotopes are present in a much higher abundance than heavier isotopes, variations in these ratios can appear at the $4^{\text {th }}-6^{\text {th }}$ decimal point. For this reason, isotopic variations have 
historically been expressed in the units per mille (\%o, parts per thousand). As this unit is dimensionless, however, it presents limitations in expressing orders of magnitude of difference between isotopic ratios (ie. milli, micro, nano). For this reason, the use of the unit Urey, in which 1 milliUrey (mUr) is equivalent to $1 \%$, has been proposed[46]. These units will be utilized throughout this thesis.

\subsubsection{Isotope ratio mass spectrometry}

As different isotopes of carbon present different atomic masses, it is possible to detect the stable carbon isotopic ratios using mass spectrometry. A standard mass spectrometer can operate on select ion monitoring (SIM) mode in which a single detector scans simultaneously for the presence of selected masses. This can, at best, detect ${ }^{13} \mathrm{C}$ enrichment levels of 0.5 atom percent excess (APE). However, at the natural abundance level most variations in ${ }^{13} \mathrm{C}$ enrichment are within the range of 1.0343-1.1167 APE[42]. As a result, a standard mass spectrometer simply cannot detect variations in stable carbon isotope ratios that are present at the natural abundance level. For this reason, a specialized mass spectrometer known as an isotope ratio mass spectrometer (IRMS) must be utilized. An IRMS is an extremely sensitive and precise device capable of detecting isotopic ratios as low as five to six significant figures. Unlike standard mass spectrometers, an IRMS possesses multiple Faraday cup detectors. Each Faraday cup is situated to detect only one specific isotopomer. When analyzing the isotopic ratios of carbon, samples are combusted into $\mathrm{CO}_{2}$ for isotopic analysis. As such, one Faraday cup is utilized to detect specifically one of three carbon dioxide isotopomers, ${ }^{12} \mathrm{C}^{16} \mathrm{O}_{2},{ }^{13} \mathrm{C}^{16} \mathrm{O}_{2}$, or ${ }^{12} \mathrm{C}^{18} \mathrm{O}_{2}(m / z: 44,45$ and 46, respectively). Each detector scanning particularly for only one $\mathrm{m} / \mathrm{z}$ ion allows this apparatus to obtain extremely precise isotopic measurements at the expense of the flexibility of analyzing multiple ion masses allowed for by standard mass spectrometers[44,45].

\subsubsection{Carbon isotopic fractionation in the food supply}

The unique photosynthetic mechanisms by which different plant types fixate carbon is a key source of carbon isotopic variation in the food supply (Figure 1.7.5.1). There are three main plant types: $\mathrm{C} 3, \mathrm{C} 4$, and Crassulacean acid metabolism (CAM) plants. C3 plants only utilize the Calvin cycle to fix carbon[47]. These plants initiate their photosynthetic pathway in the mesophyll cells through the reaction of atmospheric $\mathrm{CO}_{2}$ with ribulose 1,5-bisphosphate (RuBP), which is catalyzed by the enzyme ribulose bisphosphate carboxylase (Rubisco). This reaction 
results in a 3 carbon product known as 3-phosphoglyceric acid. It is crucial to note that the enzyme Rubisco has a preference for ${ }^{12} \mathrm{CO}_{2}$ over ${ }^{13} \mathrm{CO}_{2}$. This results in a higher amount of ${ }^{12} \mathrm{CO}_{2}$ being utilized and increased amount of ${ }^{13} \mathrm{CO}_{2}$ diffusing out of the stomata pore, located proximally to this initial reaction. As a result, the tissue of C3 plant types is typically relatively deplete in ${ }^{13} \mathrm{C}$ in comparison to $\mathrm{C} 4$ plant types (-20 mUr to $-35 \mathrm{mUr}$ vs. -7 to $-17 \mathrm{mUr}$, respectively[48,49]. C3 plants make up $>250,000$ plant and algal species[50], including safflower, soybean, and rice found in the food supply.

In contrast to $\mathrm{C} 3$ plants, when $\mathrm{C} 4$ plants fixate carbon, they initially undergo the Hatch-Slack cycle prior to participating in the Calvin cycle[51,52]. The Hatch-Slack cycle initially begins in the mesophyll where $\mathrm{CO}_{2}$ reacts with phosphoenolpyruvate to produce oxaloacetate, a 4 carbon product. This reaction is catalyzed by the enzyme phosphoenolpyruvate carboxylase (PEP C), which preferences ${ }^{12} \mathrm{CO}_{2}$ over ${ }^{13} \mathrm{CO}_{2}$ to a much lesser extent than Rubisco. Oxaloacetate is then converted into malate which is moved to the bundle sheath cells. Here, malate is broken down into pyruvate and $\mathrm{CO}_{2}$ which will interact with Rubisco and proceed through the Calvin cycle. The bundle sheath cells allow for much less diffusion and escape of $\mathrm{CO}_{2}$ than the mesophyll cells. Further, most of the $\mathrm{CO}_{2}$ that does manage to escape from the bundle sheath cells can be fixed by PEP $\mathrm{C}$ in the mesophyll before diffusing out of the plant cell. These processes allow for less discrimination of ${ }^{13} \mathrm{CO}_{2}$ and therefore a higher ${ }^{13} \mathrm{C}$ enrichment in the tissues of $\mathrm{C} 4$ plants in comparison to C3 plants[43,49,50,53]. C4 plants make up the minority of plant species in our food supply and would include foods such as corn, sugar cane and millet[54].

CAM plants can utilize a C3 photosynthetic pathway or a C4-like photosynthetic pathway in which photosynthetic reactions are predominantly temporally separated as opposed to spatially. As a result, the tissues of these plants typically display a ${ }^{13} \mathrm{C}$ enrichment that varies between the values achieved by $\mathrm{C} 3$ and $\mathrm{C} 4$ plants. Common examples of CAM plants within the food supply would include tequila agave and pineapple[55].

Substances derived from marine environments (ie. marine fish) also represent a source of carbon isotopic variation within the food supply. Although, like terrestrial plants, the vast majority of marine plants are of the $\mathrm{C} 3$ type, they tend to exhibit a higher ${ }^{13} \mathrm{C}$ enrichment than $\mathrm{C} 3$ terrestrial plants. Atmospheric $\mathrm{CO}_{2}$ dissolves in the ocean as bicarbonate, which is subsequently taken up 
by plants, algae, and phytoplankton for photosynthesis. The dissolution of $\mathrm{CO}_{2}$ into bicarbonate in ocean water is a process that favors ${ }^{13} \mathrm{C}$, resulting in a higher ${ }^{13} \mathrm{C}$ enrichment in ocean bicarbonate relative to atmospheric $\mathrm{CO}_{2}[56]$. Further, $\mathrm{CO}_{2}$ diffuses more slowly in water than in air and as such these plants have less opportunity to discriminate against ${ }^{13} \mathrm{CO}_{2[43]}$. This results in a ${ }^{13} \mathrm{C}$ enrichment greater than can range between $\mathrm{C} 3$ and $\mathrm{C} 4$ plant types $[54,57,58]$ 

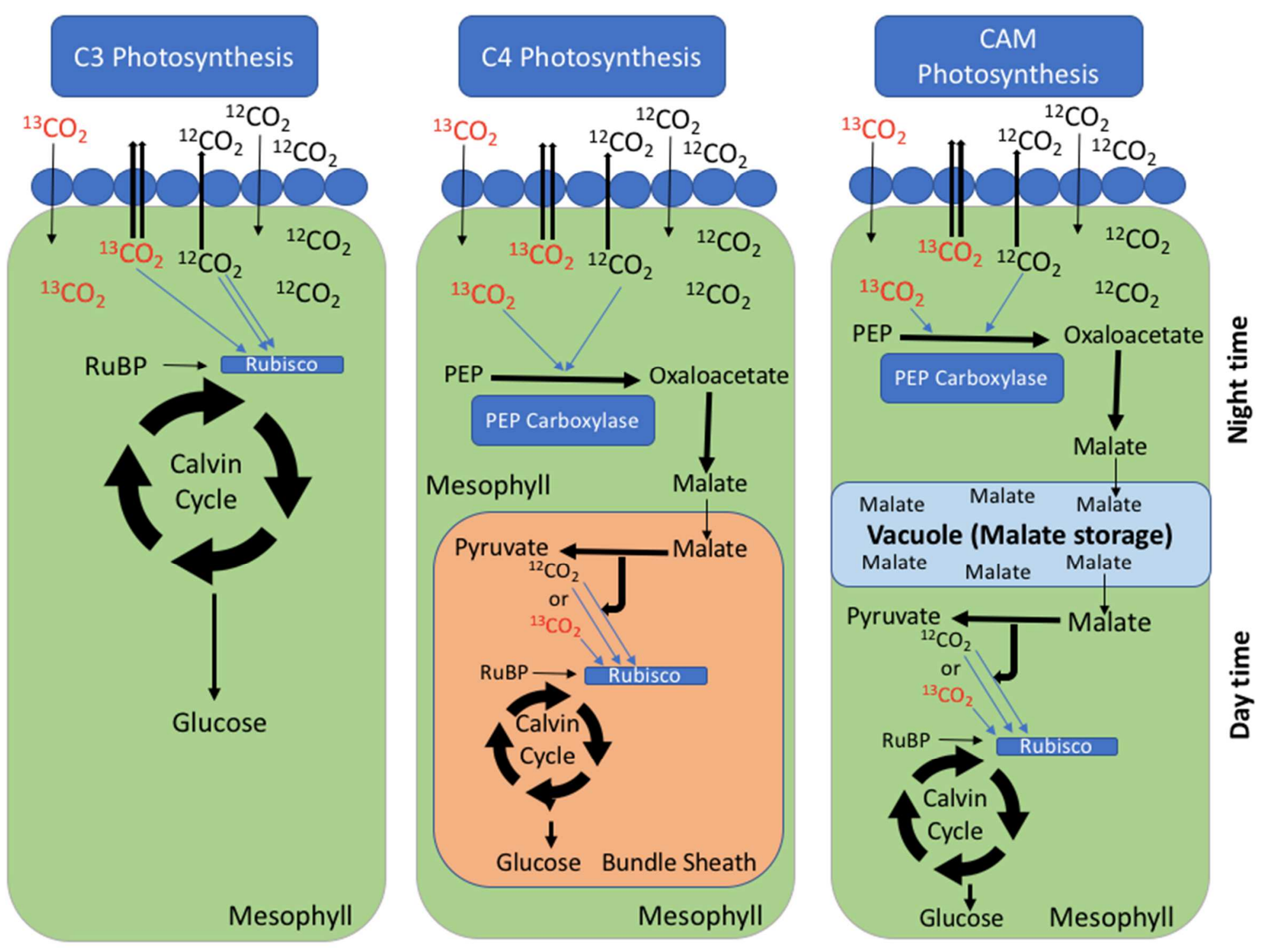

Figure 1.7.5.1 The different modes of photosynthesis of plants within the food supply 


\subsubsection{Natural abundance compound-specific isotope analysis of fatty acids}

Through coupling the precision of an isotope ratio mass spectrometer to the resolving power of a gas chromatograph, individual fatty acids can be separated and isotopically analyzed[44,45,59].

As a result, unique carbon isotopic ratios can serve as a tracer to identify the origin of fatty acids. Indeed, $\delta^{13} \mathrm{C}$ values of fatty acids have been utilized as a biomarker to track ecological food webs[60,61]. In the field of archeology, this approach has been applied to aid in reconstructing the origin of diets consumed in prehistoric times[62,63]. Further, it has also been useful for identifying the authenticity of high-quality commercially sold oils, as $\delta^{13} \mathrm{C}$ values of fatty acids may be altered when fraudulent oils are added to the mixture[64,65]. A useful review of these applications can be found here[44]. Despite this widespread use, CSIA by IRMS has been applied to a much lesser extent to the study of mammalian fatty acid origin and metabolism.

Through switching infants onto controlled, isotopically unique diets, CSIA has been applied sparingly to demonstrate synthesis of ARA[66] and DHA[67]. Rhee et al (1997) have shown that upon consumption of a controlled diet for 5 weeks, human serum fatty acid $\delta^{13} \mathrm{C}$ values are markedly altered and reflective of their provided diet[68]. Recently, through the use of CSIA of fatty acids and two- end member mixing models, our group assessed the contribution of DHA retroconversion to EPA concentrations in rats[69]. Excluding a very recent study conducted by our group[70], CSIA by IRMS has not been applied to answer complex questions pertaining to the origin of brain fatty acids. As the mentioned applications reveal, fatty acids from unique food sources that possess different ${ }^{13} \mathrm{C}$ enrichments can serve as tracers to study the origin of fatty acids. This can, therefore, provide a cost-efficient means to track the long-term origin and metabolism of brain fatty acids through the use of chronic feeding studies while negating the need to purchase costly synthesized fatty acid tracers.

\subsection{The fat-1 mouse model}

Dietary intervention studies are a common approach utilized to study the potential physiological roles of n-3 PUFA in animals. When applying this approach, however, it is impossible to keep all dietary components constant between treatment and control diets when changing dietary fat composition. The introduction of n-3 PUFA to a diet changes not only the n-6 to n-3 PUFA ratio, but also the amount of monounsaturated and saturated fatty acids along with their associated 
antioxidants, vitamins and bioactive compounds, even when the absolute amount of fat is held constant. This consequently makes the results of dietary intervention studies difficult to interpret. To address this issue, Kang et al (2004) developed the fat-1 transgenic mouse. This mouse model expresses the nematode Caenorhabditis elegans n-3 desaturase gene allowing for conversion of n-6 to n-3 PUFA, a feat mammalian species do not possess[71]. As a result, when fed a diet rich in n-6 PUFA but deficient in n-3 PUFA, these animals display tissue n-3 PUFA concentrations much higher than their wild-type littermates[71]. Using this model can allow for the feeding of one experimental diet to wild-type and fat- 1 mice while resulting in markedly different tissue fatty acid profiles, avoiding the confounding factors associated with the use of two separate diets. Prior to introducing this transgene into mice, it was expressed in a variety of mammalian cell lines including neurons[72], cardiac myocytes[73], endothelial cells and cancer cell lines[74,75].

The genetic sequence of the fat- 1 gene suggests that it is a transmembrane protein localized to the cell endoplasmic reticulum with both its $\mathrm{C}$ and $\mathrm{N}$ terminal domains facing the cytosol[76]. The $n-3$ desaturase has the ability to utilize 16-20 carbon substrates with a preferences for substrates with $n-6$ double bonds[77]. It catalyzes the production of n-3 PUFA via the addition of a double bond three carbons from the methyl end of fatty acids (Figure 1.8.1). To generate fat-1 mice, the Caenorhabditis elegans n-3 desaturase gene was coupled to the chicken-beta actin promoter and introduced to fertilized eggs via adenovirus gene transfer[71]. Coupling to the chicken-beta actin promoter results in widespread gene expression[78]. Gene expression analyses demonstrate that the fat- 1 gene is expressed to some extent in the brain, heart, kidney, liver, muscle, rectum, spleen, and testes with the heart, muscle, and testes displaying the greatest fat-1 mRNA expression[79]. Tissue (skeletal muscle, milk, erythrocyte, heart, brain, liver, kidney, lung, and spleen) fatty acid analysis of fat- 1 mice fed a diet rich in n-6 PUFA and deficient in n-3 PUFA display markedly lower n-6:n-3 PUFA ratios in comparison to their wildtype littermates. While wild-type mice had tissue n-6:n-3 ratios ranging from 20-50 (with brain n-6:n-3 PUFA ratio being 4), the fat-1 mice revealed a much more balanced phenotype with tissue $\mathrm{n}-6$ to $\mathrm{n}-3$ ratios ranging from 0.7-5.7 (with brain $\mathrm{n}-6$ to $\mathrm{n}-3$ PUFA ratio being 0.8)[71]. Along with a balance n-6/n-3 PUFA ratio the fat- 1 mouse also appears to have a characteristic lipidomic signature in comparison to wild-type animals fed n-3 PUFA low, n-6 PUFA rich $\operatorname{diets}[80]$. 
Linoleic Acid

18:2n-6
Alpha linolenic Acid

18:3n-3

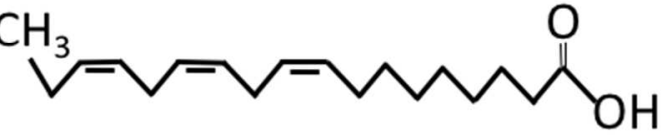

Figure 1.8.1- Enzymatic conversion of n-6 PUFA to n-3 PUFA via the n-3 desaturase enzyme in fat-1 mice 
The fat-1 mouse has been utilized to assess the effects of increased n-3 PUFA tissue concentration in various disease conditions. In models of neuroinflammation, fat-1 mice fed a $10 \%$ safflower oil diet display lower proinflammatory cytokine and chemokine gene expression, decreased markers of glial cell activation along with decreased expression of ARA cascade enzymes compared to wild-type mice[81,82]. The fat-1 mouse has also demonstrated increased hippocampal neurogenesis, increased dendritic spine density, and improvements in spatial memory[83]. The fat-1 mouse also appears to promote healing upon spinal chord injury[84], display reduced markers of melanoma[75], and be more protected against colitis[85], ovariectomy-induced bone loss[86], and atherosclerosis [87].

Previously, Orr et al (2014) assessed if the addition of n-3 PUFA to an n-6 PUFA rich diet could potentiate brain DHA concentrations in fat-1 mice beyond the levels achieved by endogenous synthesis via the fat-1 n-3 desaturase[88]. Interestingly, no difference in either total brain DHA or DHA esterified to phospholipids was noted, regardless of if the fat- 1 animals were fed a $10 \%$ safflower oil diet or an $8 \%$ safflower oil $2 \%$ fish oil diet. In both cases, animals acquired total brain DHA concentrations of approximately $12000 \mathrm{nmol} / \mathrm{g}$. Although these animals achieve similar brain DHA levels, it remains unclear if fat-1 mice fed the fish-oil containing diet preferentially obtain their brain DHA via synthesis from dietary n-6 PUFA or through the uptake of dietary preformed DHA. A key factor that has limited our group from resolving this question is the lack of reliable, cost-efficient methodologies available to study the origin of brain fatty acids. Here, we demonstrate the power of CSIA by IRMS to successfully trace the origin of brain DHA in this complex model. 


\section{Chapter 2:}

Objective and Hypothesis 


\subsection{Objective}

The objective of this study is to apply natural abundance compound-specific isotope analysis to determine the main source of brain DHA in fat-1 mice fed a diet rich in both n-3 and n-6 PUFA.

\subsection{Hypotheses}

1)The carbon isotopic signature of brain DHA will reflect the carbon isotopic signature of its dietary origin.

2)The fat-1 mouse will obtain its brain DHA through the synthesis of n-3 PUFA from n-6 PUFA. 


\section{Chapter 3 \\ Applying Stable Carbon Isotopic Analysis at the Natural Abundance level to Determine the Origin of Docosahexaenoic Acid in the Brain of the Fat-1 Mouse}

Contributions: I, along with my supervisor (Dr. Richard Bazinet), decided upon the design, focus, and direction of this project. I conducted all laboratory work and analyses with exceptions noted below. Dr. Kathryn Hopperton assisted me with mouse colony maintenance and animal sacrifice. R.J Scott Lacombe (PhD candidate) provided me with expertise and assistance with gas-chromatography isotope ratio mass spectrometry measures. The GC-IRMS utilized was operated by Ashley St.Pierre at the Sick Kids Analytical facility of Bioactive Molecules. I independently analyzed all data retrieved. I have also prepared a first author manuscript for submission. 


\subsection{Abstract:}

A key factor limiting the study of the origin and metabolism of brain fatty acids is the lack of cost-efficient methods available to trace fatty acids. Here, through the application of compoundspecific isotope analysis (CSIA), a novel, cost-efficient method, we successfully differentiated between brain DHA originating directly from dietary omega (n)-3 polyunsaturated fatty acids (PUFA), and brain DHA biochemically synthesized to determine the origin of brain DHA in fat1 mice. Fat-1 mice and their wild-type littermates were either weaned onto n-6 PUFA rich, n-3 PUFA deficient diets or diets rich in both n-3 and n-6 PUFA. Isotopic analysis of fatty acid methyl esters from brain and liver tissue was conducted via gas chromatography-isotope ratio mass spectrometry. Our data demonstrates that in the presence of n-3 and n-6 PUFA, fat-1 mice obtain their brain DHA predominately from dietary n-3 PUFA sources. This study reflects the first application of natural abundance CSIA by IRMS to a complex multivariate model to determine the origin of brain fatty acids. 


\subsection{Introduction}

Docosahexaenoic acid (DHA; 22:6 n-3) is an omega(n)-3 polyunsaturated fatty acid (PUFA) that mammals are unable to synthesize de novo and therefore must obtain via dietary means. DHA is found abundantly in foods such as fatty fish or synthesized upon consumption of its dietary precursor, alpha-linolenic acid (ALA; 18:3n-3). DHA is the most abundant n-3 PUFA in the brain and makes up approximately $10 \%$ of brain fatty acids $[89,90]$. It is highly enriched within neuronal membranes and is essential for normal neurodevelopment and the maturation of visual acuity[13-15]. More recently, emerging evidence has suggested that low consumption of n-3 PUFA is associated with neurological and psychiatric disease[17,91,92]. Further, DHA derived bioactive mediators have also been suggested to play a key biological role in the resolution of inflammation[24].

The surmounting evidence supporting a crucial physiological role of DHA in the brain[12,14,17,93] has motivated interest in determining the origin and metabolic fate of DHA and its dietary precursor, ALA. Despite this interest, a host of pertinent questions concerning brain DHA metabolism, including identifying the brain's preferred source of DHA, have yet to be elucidated. A limiting factor that has prevented this question from being resolved is the lack of reliable and cost-efficient methodologies presently available to trace the metabolic fate of DHA in vivo. Currently, the administration of stable or radio labelled fatty acids is a common method applied to trace the incorporation and metabolism of fatty acids in tissues. However, this approach does present limitations. Firstly, labelled fatty acids are costly, making it infeasible to conduct chronic studies that label all fatty acids of interest. Moreover, studies that have orally administered labelled tracers to rodents have noted that a large proportion of the provided tracer that is not beta-oxidized is sequestered in the adipose tissues[41], leaving the metabolic fate of these tracers unavailable for study at acute time points. It is also crucial to note that when these tracers are administered orally, data obtained at acute time points may only reflect their postprandial biochemical fate, as opposed to the metabolism and turnover of the total fatty acid pool[5]. Alternatively, it is possible to administer these tracers intravenously either through bolus injections or steady-state infusion methods. Although this approach does allow for whole body fatty acid synthesis rates to be estimated, it is still limited in scope to acute time points that may not necessarily reflect the chronic metabolism and turnover of the fatty acid of interest. Clearly, 
novel methods to chronically trace the incorporation and turn-over of tissue fatty acids are needed.

Natural abundance compound specific isotope analysis (CSIA) by isotope ratio mass spectrometry (IRMS) is a highly precise methodology that can detect naturally occurring variations in stable carbon isotopes[94,95]. As a result of the unique carbon fixation pathways that different plant types undergo, a natural variation in ${ }^{13} \mathrm{C}$ enrichment $\left(\delta^{13} \mathrm{C}\right)$ exists within the food supply. Tissue from $\mathrm{C} 3$ plant types that undergo carbon fixation via the Calvin cycle tend to be less ${ }^{13} \mathrm{C}$ enriched (-23 to $-32 \mathrm{mUr}$ ) compared to $\mathrm{C} 4$ plant types which fix carbon through the Hatch-Slack pathway (-10 to $-16 \mathrm{mUr}$ ) [57]. ${ }^{13} \mathrm{C}$ enrichment is also distinguished in aquatic environments (-16 to $-22 \mathrm{mUr}$ ) [57]. As a result, fatty acids derived from terrestrial $\mathrm{C} 3$ or $\mathrm{C} 4$ plants, such as ALA, will exhibit isotopic signatures that differ not only from one another, but also from fatty acids such as DHA, derived from marine sources [53,96,97]. Through coupling a gas-chromatograph (GC) to the remarkable precision of an IRMS, slight isotopic variations in individual fatty acids can be detected and serve as a marker to trace the incorporation of dietary lipids into the brain without the need of expensive labelled fatty acids. Recently, for the first time, our group explored this approach as a novel, cost-efficient means to trace the origin of brain PUFA [70]. In the present study, we applied this method to determine the source of brain DHA in fat-1 mice, a complex question that would have been previously infeasible to answer [88] without the application of this method. Unlike typical mammalian species, the fat-1 mouse expresses the C. elegans fat-1 gene that encodes for an n-3 desaturase enzyme which drives the endogenous conversion of n-6 PUFA to n-3 PUFA [71,98]. It remains unclear if fat-1 mice fed an n-3 and n-6 PUFA rich diet preferentially obtain their brain DHA through the enzymatic conversion from n-6 PUFA or via uptake of dietary preformed DHA. Here, through the application of CSIA by IRMS, we successfully differentiated between brain DHA originating directly from fish oil, and brain DHA biochemically synthesized by wild-type or transgenic enzymes. Our findings demonstrate that when fed diets rich in n-3 and n-6 PUFA, fat- 1 mice obtain their brain DHA solely from preformed DHA. 


\subsection{Methods}

\subsubsection{Animals}

All procedures were carried out in accordance with the policies set by the Canadian Council on Animal Care and the Regulations of Animals for Research Act in Ontario. Experimental protocols were approved by the Animal Ethics Committee at the University of Toronto, Canada (protocol number 2016/17: 20011720). Male fat-1 mice were generously donated to our group by Dr. David Ma (University of Guelph, ON). Female C57BL/6 x C3H breeders were obtained from Charles River Laboratories (CRL; Saint-Constant, Quebec, Canada). Upon arrival to our facility, mice were switched to a $10 \%$ safflower oil (SO) diet and left to acclimatize for two weeks. Our facility maintained a constant light cycle $(12 \mathrm{~h} \mathrm{light} / 12 \mathrm{~h}$ dark $)$, temperature $\left(21^{\circ} \mathrm{C}\right)$ and humidity, with ad libitum access to food and water. After this acclimatization period, 3 female $\mathrm{C} 57 \mathrm{BL} / 6 \mathrm{x} \mathrm{CH} 3$ mice were placed into harems with one male fat-1 mouse for breeding. Harems were maintained on the SO diet. Mothers were maintained on the SO diet throughout breeding, pregnancy, and lactation. Female fat-1 pups along with their wild-type littermates bred from these harems were utilized in this study. To minimize genetic redundancy, only one wild-type and one fat-1 animal were selected from each litter. Methods employed to genotype the mice utilized in this study have previously been reported by our group [81]. Briefly, $2 \mathrm{~mm}$ mouse tail clippings were placed into a solution containing $300 \mu \mathrm{l}$ of cell lysis buffer and $20 \mu \mathrm{l}$ of $10 \mathrm{mg} / \mathrm{mL}$ proteinase $\mathrm{K}$, and left to digest overnight in a $55{ }^{\circ} \mathrm{C}$ water bath. The following day, digested samples were pelleted via centrifugation (20 min at 12900 relative centrifugal force (g)). DNA was subsequently precipitated by pouring the resulting supernatant into $400 \mu \mathrm{l}$ of isopropanol and pelleted via centrifugation (10 min at $12900 \mathrm{~g}$ ). The supernatant was removed and DNA pellet was left to dry for $30 \mathrm{~min}$. Forty $\mu \mathrm{l}$ of $\mathrm{x} 1$ Tris EDTA buffer was added to the DNA pellet and placed in a $55^{\circ} \mathrm{C}$ water bath for $10 \mathrm{~min}$. In preparation for PCR, $1.5 \mu$ of DNA was added to commercial master mix (ThermoScientific, Waltham, MA, USA) which was prepared according to the manufactures instructions. Thermocycler conditions were as follows: 2 min at $95^{\circ} \mathrm{C}, 30$ cycles for $30 \mathrm{~s}$ each at $94^{\circ} \mathrm{C}, 30$ cycles for $30 \mathrm{~s}$ each at $55^{\circ} \mathrm{C}, 30$ cycles for $1 \mathrm{~min}$ each at $72{ }^{\circ} \mathrm{C}$ and completed with a final elongation cycle for $10 \mathrm{~min}$ at $72{ }^{\circ} \mathrm{C}$. 
At three weeks of age, mice were either maintained on the SO diet or weaned onto an $8 \%$ safflower oil, $2 \%$ fish oil (SOFO) diet. As lipids derived from the safflower have a distinct $\delta^{13} \mathrm{C}$ value from those derived from fish oil, the brain source of DHA in animals that consume these diets can be determined through comparing $\delta^{13} \mathrm{C}$ values of DHA to the $\delta^{13} \mathrm{C}$ value of the fatty acids within their respective diets. A separate subset of animals were also weaned onto either a $10 \%$ corn oil diet $(\mathrm{CO})$ or an $8 \%$ corn oil, $2 \%$ fish oil (COFO) diet. As corn oil exhibits a unique $\delta^{13} \mathrm{C}$ value compared to both safflower and fish oil, this subset of animals allowed for validation of our findings. Mice were maintained on these diets for 9 weeks (until 12 weeks of age) at which point they were euthanized via cardiac puncture under terminal anesthesia as described below. A separate subset of wild-type and fat-1 mice were euthanized at 3 weeks of age, prior to weaning for baseline measurements. Upon sacrifice tissues of interest were collected, flashfrozen in liquid nitrogen and stored at $-80^{\circ} \mathrm{C}$.

\subsubsection{Diets}

Diets were obtained from Research Diets Inc (New Brunswick, NJ, USA) and differed only in fatty acid composition, but not amount of total fat. Each diet was modified from the AIN 93-G rodent diet and contained 10\% fat, 59\% carbohydrates and $21 \%$ protein $(\mathrm{w} / \mathrm{w})$. The SO diet contained 10\% safflower oil (D04092701). The SOFO diet contained 8\% safflower and 2\% menhaden oil (D04092702). The CO contained 10\% corn oil (D16053113). The COFO diet contained 8\% corn oil, $2 \%$ menhaden oil (D16053114). Fatty acid composition of diets was determined in-house. Total lipids were extracted using methods adapted from Folch et al [99]. Fatty acid composition of lipid extracts was analyzed by GC-flame ionization detection as described below and is presented in Table 3.3.2.1. The most abundant fatty acid present in each diet was LA making up 71,58,55 and $45 \%$ of the SO, SOFO, CO, COFO diets respectively. Arachidonic acid was not detected in SO or CO diet but was made up approximately $0.2 \%$ and $0.3 \%$ of the SOFO and COFO diets respectively. DHA was not detected in either the CO or SO diet but made up approximately $1.2 \%$ and $1.4 \%$ of fatty acids in the SOFO and COFO diets. Other n-3 PUFA present included ALA which made up 0.2, 0.5, 0.9, and 1\% of fatty acids in the $\mathrm{SO}, \mathrm{SOFO}, \mathrm{CO}$, and COFO diets respectively and EPA which was not detected in the $\mathrm{SO}$ and CO diet but made up $2.7 \%$ and $2.4 \%$ of the SOFO and COFO diets. DPA n-3 was not detected in the $\mathrm{SO}$ and $\mathrm{CO}$ diet but was present at approximately $0.4 \%$ in both the SOFO and COFO diet. 
Fatty acid composition

\begin{tabular}{|c|c|c|c|c|}
\hline $14: 0$ & 0.17 & 2.09 & 0.08 & 2.03 \\
\hline $16: 0$ & 7.09 & 9.18 & 12.43 & 13.84 \\
\hline 18:0 & 2.48 & 2.57 & 1.65 & 1.96 \\
\hline $16: 1 n-7$ & 0.10 & 2.69 & 0.12 & 2.58 \\
\hline $18: 1 n-7$ & 0.71 & 1.15 & 0.58 & 1.03 \\
\hline $18: \ln -9$ & 15.18 & 13.44 & 27.44 & 23.41 \\
\hline $20: 1 n-9$ & 0.18 & 0.37 & 0.30 & 0.50 \\
\hline $18: 2 n-6$ (LA) & 71.37 & 58.77 & 55.14 & 45.16 \\
\hline$\delta^{13} C_{L A}$ & $-31.86 m U r$ & $-30.67 m U r$ & $-16.51 m U r$ & $-16.15 m U r$ \\
\hline $20: 4 n-6$ (ARA) & n.d. & 0.16 & n.d. & 0.25 \\
\hline 18:3n-3 (ALA) & 0.24 & 0.45 & 0.89 & 0.99 \\
\hline $20: 5 n-3$ (EPA) & n.d. & 2.67 & n.d & 2.44 \\
\hline$\delta^{13} C_{E P A}$ & - & $-24.28 m U r$ & - & $-23.91 m U r$ \\
\hline $22: 5 n-3$ (DPA) & n.d. & 0.35 & n.d. & 0.42 \\
\hline 22:6n-3 (DHA) & n.d. & 1.22 & n.d. & 1.39 \\
\hline$\delta^{13} C_{D H A}$ & - & $-21.99 m U r$ & - & $-21.98 m U r$ \\
\hline
\end{tabular}

Table 3.3.2.1: Fatty acid composition and $\delta^{13} \mathrm{C}$ values of key dietary fatty acids Fatty acid composition expressed as relative percent of total fatty acids and $\delta^{13} \mathrm{C}$ values of key fatty acids in the $10 \%$ fat diets. Fatty acid analysis was conducted in triplicate and values are expressed as means. Other fatty acids present in quantities $\leq 0.5 \%$ are not displayed. Abbreviations: ARA, arachidonic acid; ALA, $\alpha$-linolenic acid; EPA, eicosapentaenoic acid; DHA, docosahexaenoic acid; DPA, docosapentaenoic acid; LA, linoleic acid; n.d, not detected. 


\subsubsection{Cardiac puncture}

Mice were anesthetized at 12 weeks of age through intraperitoneal injection of $20 \mu \mathrm{l} / \mathrm{g}$ of Avertin (0.25 g of 2,2,2 tribromoethanol, $0.5 \mathrm{~mL}$ of 2-methyl-2-butanol, $20 \mathrm{~mL} \mathrm{dH}_{2} \mathrm{O}$, Sigma Aldrich, St-Louis, MO, USA). To confirm that physical sensation was absent, toe-pinching was preformed to monitor pedal-reflexes. Once mice no longer responded to toe-pinching, they were placed dorsally and a 23 -gauge needle attached to a $1 \mathrm{~mL}$ syringe was gently inserted laterally into their left chest wall. Once the heart was punctured the syringe was slowly aspirated to draw blood.

\subsubsection{Lipid extraction}

Total lipids were extracted by methods adapted from Folch et al[99]. Diet, brain and liver tissue were homogenized in $4 \mathrm{~mL}$ of chloroform and $2 \mathrm{~mL}$ of methanol. Heptadecanoic acid (17:0) and docosatrienoic ethyl ester (22:3 n-3) (Nu Chek Prep, Elysian, MN) in chloroform were added as internal standards and the solution was left to incubate overnight at $4{ }^{\circ} \mathrm{C}$. The following day, $1.75 \mathrm{~mL}$ of a $0.88 \%$ solution of potassium chloride was added to induce phase separation. The sample then underwent centrifugation for $10 \mathrm{~min}$ at $500 \mathrm{~g}$, and the bottom chloroform layer of the solution was extracted and transferred into a separate glass tube.

\subsubsection{Lipid methylation and gas chromatography}

In order to confirm the fatty acid phenotype of fat-1 and wild-type animals consuming their respective diets, we analyzed the fatty acid composition of brain and liver tissue[100]. Total lipid extracts (TLE) of brain and liver were evaporated under nitrogen and reconstituted with hexane. $300 \mu \mathrm{l}$ of TLE was methylated using $1 \mathrm{~mL}$ of $14 \%$ boron trifluoride in methanol (Sigma-Aldrich) and heated at $100{ }^{\circ} \mathrm{C}$ for one $\mathrm{h}$ to produce fatty acid methyl esters (FAME). Samples were left to cool for $15 \mathrm{~min}$, after which $1 \mathrm{~mL}$ of reconstituted distilled $\mathrm{H}_{2} \mathrm{O}$ was added to terminate the reaction along with $1 \mathrm{~mL}$ of hexane. Samples were subsequently centrifuged at $500 \mathrm{~g}$ for $10 \mathrm{~min}$ and the top organic layer was extracted, evaporated down under nitrogen and reconstituted in 1 $\mathrm{mL}$ of hexane. Total fatty acids were then quantified via gas chromatography using a Varian 430 GC (Bruker, Billerica, MA, USA) equipped with a flame ionization detector and a SP-2560 capillary column $(100 \mathrm{~m}, 0.25 \mathrm{~mm}$ inner diameter, $0.2 \mu \mathrm{m}$ film thickness; Supleco by SigmaAldrich, Bellefonte, PA, USA) as previously described in detail by our group here[70]. Briefly, 1 $\mu 1$ of prepared sample was injected in splitless mode by a Varian CP-8400 auto-sampler. The 
injection port was set at $250^{\circ} \mathrm{C}$. FAME were eluted using the following temperature program: Originally set to $60^{\circ} \mathrm{C}$ and kept at this temperature for $2 \mathrm{~min}$, increased at $10^{\circ} \mathrm{C} / \mathrm{min}$ and held at $170{ }^{\circ} \mathrm{C}$ for $4 \mathrm{~min}$, increased at $6.5^{\circ} \mathrm{C} / \mathrm{min}$ to $175^{\circ} \mathrm{C}$, increased at $2.6^{\circ} \mathrm{C} / \mathrm{min}$ to $185^{\circ} \mathrm{C}$, increased at $1.3{ }^{\circ} \mathrm{C} / \mathrm{min}$ to $190^{\circ} \mathrm{C}$, and finally increased $8{ }^{\circ} \mathrm{C} / \mathrm{min}$ to $240^{\circ} \mathrm{C}$ and kept at this temperature for $11 \mathrm{~min}$. Peaks were identified by comparison to a reference standard (GLC$569 ; \mathrm{Nu}$ Chek Prep, Elysian, MN) and quantified through proportional comparison to internal standard (22:3n-3).

\subsubsection{Isotopic analysis of fatty acids}

Isotopic analysis was conducted on FAME via GC-IRMS (Figure 3.3.6.1) ${ }^{14}$. Briefly, FAME were separated using Trace 130 GC (Thermo Scientific, Bremen, Germany) equipped with an SP-2560 capillary column ( $100 \mathrm{~m}, 0.25 \mathrm{~mm}$ inner diameter, $0.2 \mu \mathrm{m}$ film thickness). Two $\mu 1$ of prepared FAME were injected in splitless mode. FAME were eluted using the same temperature program outlined above except in the final step the temperature was elevated at $2^{\circ} \mathrm{C} / \mathrm{min}$ to reach $240{ }^{\circ} \mathrm{C}$. Helium was utilized as a carrier gas. Separated FAME were directed to a GC Iso Link II (Thermo Scientific, Bremen, Germany) combustion furnace held at $1000{ }^{\circ} \mathrm{C}$ equipped with $\mathrm{Ni}$ and $\mathrm{Cu}$ catalyst wires. The $\mathrm{GC}$ effluent was combusted into $\mathrm{CO}_{2}$ and $\mathrm{H}_{2} \mathrm{O} . \mathrm{H}_{2} \mathrm{O}$ was removed by a Nafion water trap (DuPont, Wilmington, DE, USA) while $\mathrm{CO}_{2}$ was fed into a ConFlo IV (Thermo Scientific) continuous flow interface to enter a high precision Delta V Plus IRMS (Thermo Scientific). The IRMS was equipped with three faraday cups situated to capture the major ions $m / z 44,45$, and 46 . 
a)

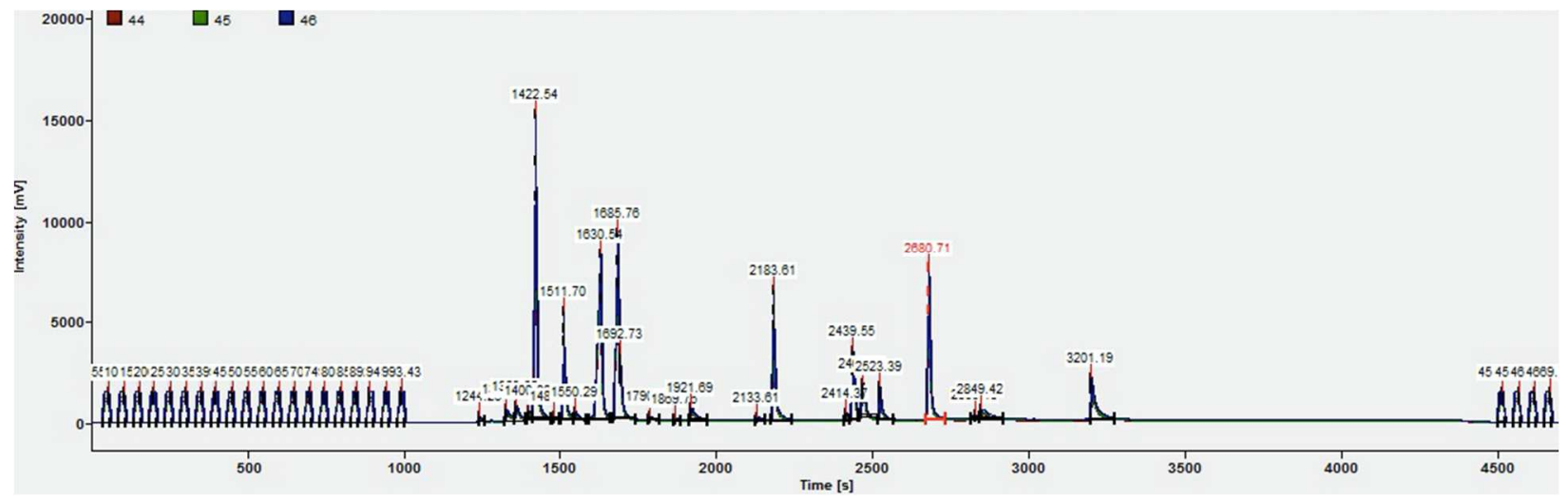

b)

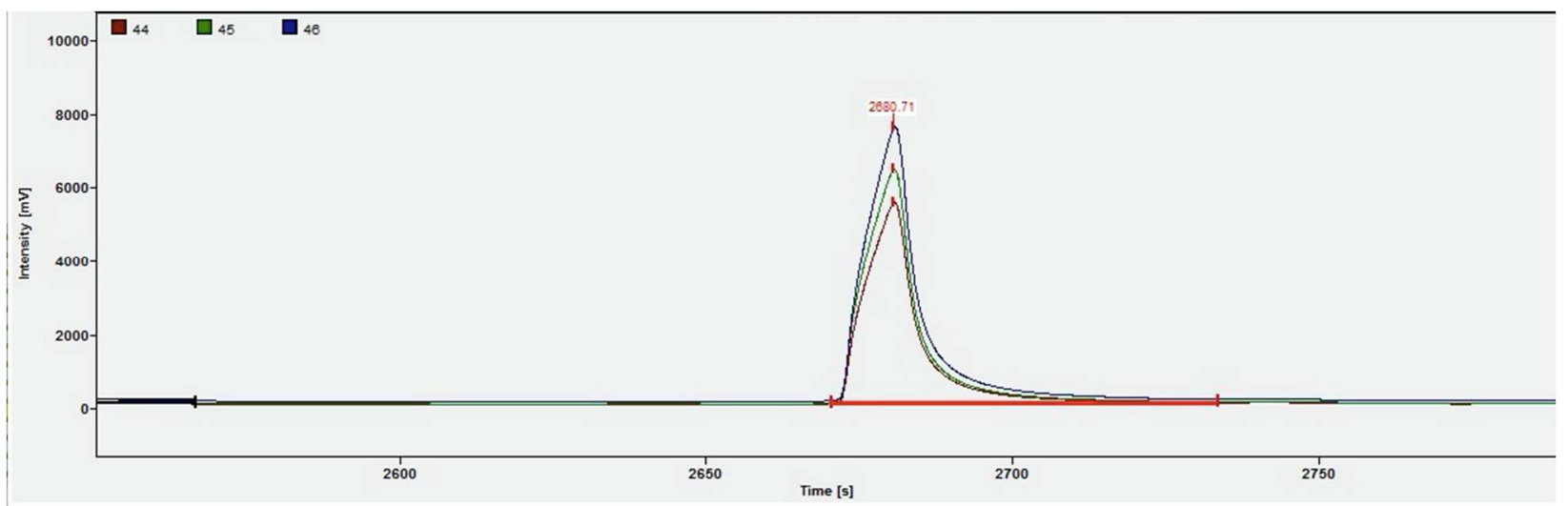

Figure 3.3.6.1: Example of a GC-IRMS chromatogram

A) GC-IRMS chromatogram of FAME derived from a mouse brain extract. Signals detected before $1000 \mathrm{~s}$ and after $4500 \mathrm{~s}$ reflect peaks of calibrating standard $\mathrm{CO}_{2}$ reference gas. B) Zoomed in view of DHA signal peak displayed in panel A. Red, green and blue traces reflect the signal peaks of $\mathrm{m} / \mathrm{z} 44,45$, and 46 respectively. Numbers above peaks reflect analyte retention times in seconds. Abbreviations: $\mathrm{CO}_{2}$, carbon dioxide; DHA, docosahexaenoic acid; FAME, fatty acid methyl ester; GC-IRMS, gas chromatography- isotope ratio mass spectrometry. 
To allow for inter-laboratory comparison, raw isotopic values were converted to the Vienna Peedee Belemnite (VPDB) international isotope reference scale using multi-point linear regression. To do this, certified reference standards (USGS70, USGS 71, USGS 72; Reston Stable Isotope Laboratory - United States Geological Survey, Reston, VA) were injected over the course of each GC-IRMS sequence. Linear regression of measured values versus true values was used to formulate a normalizing equation which was subsequently applied to the measured IRMS values of all analyzed FAME reported (Figure 3.3.6.2). 


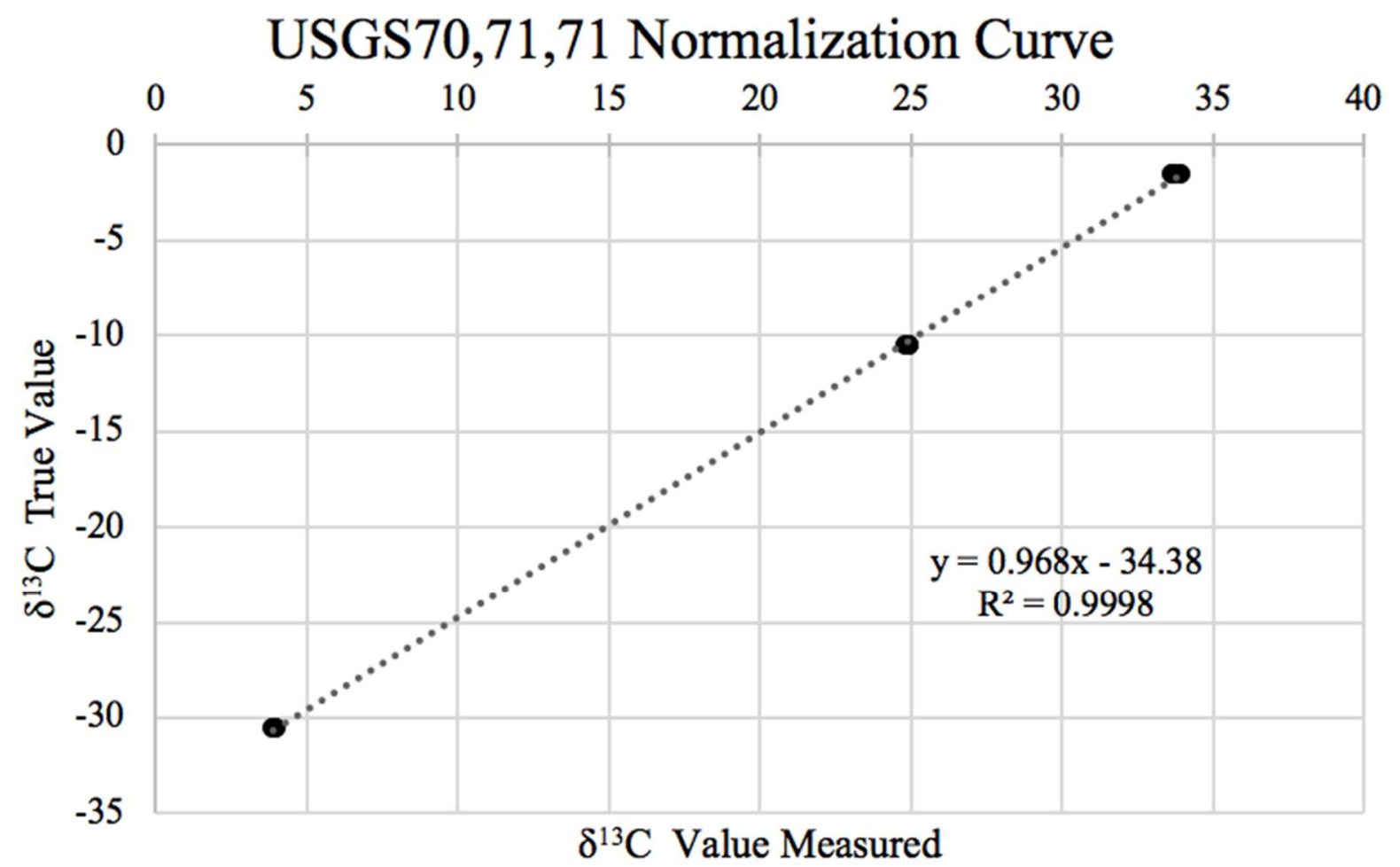

Figure 3.3.6.2: Multipoint linear normalization curve

True $\delta^{13} \mathrm{C}$ values were determined through multipoint linear normalization using 20 carbon FAME reference standards (USGS 70, 71, and 72). At least one of each USGS standard was injected with each GC-IRMS sequence run. 
It is key to note that the combustion of FAME to produce $\mathrm{CO}_{2}$ for analysis provides an isotopic value that includes not only the fatty acid of interest but also the methyl group esterified to the fatty acid upon transesterification. To correct for the methyl group contribution on the measured isotopic value, elemental analysis (EA) of carbon was conducted on free 17:0 standard and methyl ester 17:0. Samples dissolved in chloroform were loaded into tin capsules and left to dry overnight. Once the samples were completely dry, tin capsules entered a Flash 2000 Elemental Analyzer (Thermo scientific). Samples were combusted within a combustion furnace held at $1000{ }^{\circ} \mathrm{C}$ in the presence of excess oxygen. $\mathrm{CO}_{2}$ produced was then carried to the EA oxidation furnace held at $1020{ }^{\circ} \mathrm{C}$ containing a $\mathrm{CrO}_{3}$ catalyst and subsequently swept into a reduction chamber fixed at $650{ }^{\circ} \mathrm{C}$ containing a $\mathrm{Cu}$ catalyst. The resultant product was then introduced to the IRMS for isotopic analysis. To correct for the presence of atmospheric $\mathrm{CO}_{2}$ blank capsules were combusted prior to each sequence.

All raw data retrieved from EA-IRMS analysis was normalized using the same methods described above for the GC-IRMS analysis. The following rearranged mass balance equation was then applied to obtain the isotopic value of the methyl group:

$$
\text { Equation (3.3.6.1) } \delta^{13} C_{M E}=n_{F A M E} \delta^{13} C_{F A M E}-n_{F A} \delta^{13} C_{F A}
$$

Where $\delta^{13} C_{M E}, \delta^{13} C_{F A M E}$, and $\delta^{13} C_{F A}$ represents the normalized isotopic value of the methyl group, methyl ester 17:0 and FAME and free 17:0 respectively and $n_{F A M E}$ and $n_{F A}$ represents moles of carbon per mole of methyl ester 17:0 and free 17:0 respectively. Once the isotopic value of methyl group was determined, equation 2 was applied to the normalized $\delta^{13} \mathrm{C} \mathrm{FAME}$ values to correct for the presence of the esterified methyl group and obtain a $\delta^{13} \mathrm{C}$ value of solely the fatty acid of interest.

$$
\text { Equation (3.3.6.2): } \delta^{13} \mathrm{C}_{\mathrm{FA}}=\left[\left(n_{F A M E} \delta^{13} C_{F A M E}\right)-\delta 13 C_{M E}\right] / n_{F A}
$$

Where $\delta^{13} \mathrm{C}_{\mathrm{FA}}$ and $\delta^{13} C_{F A M E}$ represent the isotopic values the free fatty acid of interest and its methylated product, respectively and $\delta^{13} C_{M E}$ represents the isotopic value of the methyl group. $n_{F A}$ and $n_{F A M E}$ represent the moles of carbon per mole of free fatty acid and methylated product respectively.

All chromatograms retrieved from GC-IRMS and EA-IRMS analysis were processed using Isodat Workspace 3.0 (Thermo Scientific). All isotopic data is expressed in the units milliUrey (mUr), where $1 \mathrm{mUr}$ is equivalent to $1 \%$ relative to the VPDB international standard. 


\subsubsection{Statistics}

All values are presented as mean \pm SD. Differences in brain and liver fatty acid concentrations and $\delta^{13} \mathrm{C}$ values between the diet groups and genotypes were analyzed by two-way ANOVA with Tukey's post-hoc test applied in the case of a significant interaction. When analyzing

differences in liver $\delta^{13} \mathrm{C}$ values in SO and SOFO fed animals, a one-way ANOVA was conducted as liver DHA concentrations were too low in wild-type SO fed animals to obtain a valid $\delta^{13} \mathrm{C}$ values. 


\subsection{Results}

\subsubsection{Brain fatty acid concentration}

Brain fatty acid concentrations of baseline mice and mice fed the n-3 PUFA deficient SO diet and the n-3 PUFA containing SOFO diet were analyzed. As expected, a significant genotype $\mathrm{x}$ diet interaction effect on brain DHA concentrations was present $(F(1,22)=105.7, p<0.0001)$. Brain DHA concentrations were significantly lower in wild-type mice fed the SO diet $(n=7)$ in comparison to the wild-type SOFO fed mice $(n=6)$ and fat-1 mice fed either diet (Figure 3.4.1.1 a). There were no significant differences in brain DHA between fat- 1 animals fed the SO $(n=6)$ or SOFO diets $(n=7)$ or wild-type animals fed the SOFO diet. This is consistent with findings presented by Orr et al [88] that previously analyzed brain DHA concentrations of wild-type and fat-1 mice fed these same diets. In regards to brain ARA concentrations, there was no genotype $\mathrm{x}$ diet interaction effect or main effect of genotype present $(F(1,22)=13.94, p>0.05)$ in mice fed these diets, but a significant main effect of diet was detected $(F(1,22)=0.77, p=0.001)$ (Figure

\subsubsection{1 b).}

Whole brain fatty acid concentrations were also measured in a separate subset of wild-type and fat-1 mice weaned onto a $\mathrm{CO}$ and $\mathrm{COFO}$ diet. Consistent with the finding stated above, a significant genotype $\mathrm{x}$ diet interaction effect on brain DHA concentrations was present. Brain DHA concentrations of wild-type mice fed a CO diet $(n=4)$, deplete in DHA, were significantly lower than wild-type mice fed the COFO $\operatorname{diet}(\mathrm{n}=5)$ or fat- 1 mice fed the $\mathrm{CO}(\mathrm{n}=4)$ or $\mathrm{COFO}$

$\operatorname{diet}(n=6)$. Further, fat-1 mice fed either diet did not differ in brain DHA concentrations and also did not differ from wild-type mice fed the COFO diet (Figure 3.4.1.2 a). Once again, a significant main effect of diet on brain ARA concentrations was detected $(F(1,15)=14.00, p<0.005$,$) , in the absence of a significant genotype \mathrm{x}$ diet interaction effect or main effect of genotype.

\subsubsection{CSIA by IRMS determines the origin of brain DHA}

The fat- 1 mouse expresses the $C$. elegans fat- 1 gene that encodes for an n-3 desaturase enzyme allowing for the endogenous conversion of n-6 PUFA to n-3 PUFA (Figure 3.4.2.1)[71]. When fed a diet rich in both n-3 and n-6 PUFA, it is unclear if fat-1 mice obtain their brain DHA 
through the enzymatic conversion from n-6 PUFA or via uptake of dietary preformed DHA[88]. To resolve this question, we weaned fat-1 mice and wild-type controls onto either an n-3 PUFA low SO diet or a n-3 and n-6 PUFA rich FO diet. As the n-6 PUFA in these diets are largely derived from $\mathrm{SO}$, a $\mathrm{C} 3$ plant source, they will be more deplete in ${ }^{13} \mathrm{C}$ than the dietary n-3 PUFA which were derived from FO. Using CSIA by IRMS, we compared the $\delta^{13} \mathrm{C}$ values of dietary n-3 and n-6 PUFA to the isotopic values of brain DHA in animals consuming these diets in order to determine its dietary origin. To validate our findings, we also weaned a separate subset of mice onto an n-3 low CO diet or an n-3 and n-6 PUFA rich COFO diet. In these diets, the n-6 PUFA are largely derived from $\mathrm{CO}$ and therefore more ${ }^{13} \mathrm{C}$ enriched than the dietary $\mathrm{n}-3$ PUFA derived from FO or the n-6 PUFA present in the SO and SOFO diets. An overview of our study design is presented in Figure 3.4.2.2 a.

Mean $\delta^{13} \mathrm{C}$ values of the main dietary n-6 PUFA, LA, and n-3 PUFAs, DHA, and EPA, in each diet are displayed in Table 3.3.2.1. In both the SO and SOFO diets, the main source of LA was safflower oil, a C3 plant source. As such, the isotopic values of LA in the SO and SOFO diets were similar and more deplete in ${ }^{13} \mathrm{C}$ than $\mathrm{C} 4$ plant or marine sources $(-31.86 \pm 0.12 \mathrm{mUr}$ and $30.67 \pm 0.27 \mathrm{mUr}$, respectively). DHA and EPA, which were only present in the SOFO diet, had $\delta^{13} \mathrm{C}$ values of $-21.99 \pm 0.44 \mathrm{mUr}$ and $-24.28 \pm 0.37 \mathrm{mUr}$, respectively and were thus distinguishable from SO derived fatty acids.

To validate our findings, a separate subset of animals were weaned onto $\mathrm{CO}$ and $\mathrm{COFO}$ diets. As corn plants are of $\mathrm{C} 4$ type, fatty acids derived from this source are more ${ }^{13} \mathrm{C}$ rich than fatty acids derived from $\mathrm{C} 3$ plants like safflower. As a result, mice obtaining their brain DHA from this source will have a more positive $\delta^{13} \mathrm{C}$ value than their fat-1 littermates obtaining their brain DHA from SO. In the CO and COFO diet, LA displayed a $\delta^{13} \mathrm{C}$ of $-16.51 \pm 0.04 \mathrm{mUr}$ and -16.15 $\pm 0.14 \mathrm{mUr}$, respectively, a more enriched value than that of dietary LA present in the SO diets. $\delta^{13} \mathrm{C}$ values of DHA and EPA in the COFO diet were $-21.98 \pm 0.89 \mathrm{mUr}$ and $-23.91 \pm 0.34 \mathrm{mUr}$, respectively, a value very similar to the DHA and EPA isotopic signatures detected in the SOFO diet. This was expected as these diets had the same n-3 PUFA source: menhaden oil.

Brain carbon isotopic signatures of animals fed the $\mathrm{SO}$ and $\mathrm{SOFO}$ diets were conducted. A significant genotype $\mathrm{x}$ diet interaction effect on brain DHA $\delta^{13} \mathrm{C}$ values was present 
$(\mathrm{F}(1,22)=7.52, \mathrm{p}=0.01)$ (Figure 3.4.1 c). Wild-type mice fed the SO diet had a brain DHA $\delta^{13} \mathrm{C}$ value of $-28.81 \pm 0.35 \mathrm{mUr}$. As shown in Table 3.3.2.1, the SO diet contains $0.3 \%$ ALA. As this was the only dietary n-3 PUFA these animals were exposed to, it is likely that this trace amount of ALA present in safflower oil is a main contributor to brain DHA concentrations in these animals. This is supported by the relatively deplete brain DHA carbon isotopic value that is reflective of fatty acids derived from a C3 plant source: safflower oil. It is also likely that brain DHA within these animals was largely obtained throughout pregnancy and lactation, prior to weaning.

Fat-1 mice fed the SO diet had a very similar, but significantly different brain DHA $\delta^{13} \mathrm{C}$ value in comparison to wild-type mice fed the same diet. The mean brain DHA $\delta^{13} \mathrm{C}$ value of fat-1 animals fed the SO diet was $-27.82 \pm 0.40$ mUr. Fat-1 mice fed the SO diet had a much higher brain DHA concentration than their wild-type littermates. Therefore, since their diet is low in n-3 PUFA, fat-1 mice must have obtained a large amount of brain DHA from the conversion of dietary LA to DHA through the activity of the n-3 fatty acid desaturase.

Wild-type animals fed the SOFO diet had an average brain DHA $\delta^{13} \mathrm{C}$ value of $-24.12 \pm 0.38$ mUr. This value was significantly more enriched in ${ }^{13} \mathrm{C}$ than those animals consuming the SO diet and more closely resembled the $\delta^{13} \mathrm{C}$ value of the preformed n-3 PUFA sources within their diet. This is because wild-type mice are not capable of synthesizing n-3 PUFA from n-6 PUFA sources and therefore could not possibly obtain such high concentrations of brain DHA without the contribution of dietary n-3 PUFA. Fat-1 mice on the SOFO diet had an average brain DHA $\delta^{13} \mathrm{C}$ value of $-23.85 \pm 0.19 \mathrm{mUr}$, significantly more enriched in ${ }^{13} \mathrm{C}$ than mice fed the SO diet, but indistinguishable from wild-type mice fed the same diet. This result largely suggests that both wild-type and fat-1 animals consuming this diet obtained their brain DHA from the same source; intact dietary DHA. As wild-type mice can only obtain brain DHA from dietary n-3 PUFA, our results indicate that fat-1 mice are also obtaining their brain DHA predominately from this source, and not through the conversion of n-6 PUFA to n-3 PUFA.

Isotopic analysis was also conducted on brain ARA in the SO and SOFO fed animals (Figure 3.4.1 d). $\delta^{13} \mathrm{C}$ values of brain ARA were $-31.36 \pm 1.55 \mathrm{mUr}$ and $-31.29 \pm 2.03 \mathrm{mUr}$ for the SO fed wild-type and fat-1 mice, respectively. The $\delta^{13} \mathrm{C}$ values of brain ARA in the wild-type and 
fat-1 animals fed the SOFO diet were $-32.46 \pm 0.45 \mathrm{mUr}$ and $-32.01 \pm 0.86 \mathrm{mUr}$, respectively. There were no significant main effects or interactions present $(F(1,22)=0.13, p>0.05))$. The lack of statistical effects present pertaining to brain ARA isotopic signatures also validates this method, as it supports that the $\delta^{13} \mathrm{C}$ values obtained for brain DHA were truly a reflection of the $\delta^{13} \mathrm{C}$ values of their dietary fatty acid origin.

As mentioned, in order to further validate our findings, a subset of animals were weaned onto either a $\mathrm{CO}$ or COFO diet. The main n-6 PUFA source in these diets was corn oil, a C4 plant source. Therefore, n-6 PUFA present in these diets were distinct and more ${ }^{13} \mathrm{C}$ enriched than SO, the main n-6 PUFA source of the SO and SOFO diets. Brain DHA $\delta^{13} \mathrm{C}$ values of these animals are displayed in Figure 3.4.1.2. Consistent with our previous findings, a significant genotype $\mathrm{x}$ diet interaction effect on brain DHA $\delta^{13} \mathrm{C}$ values was present $(\mathrm{F}(1,15)=38.99, \mathrm{p}<0.0001)$. Wildtype mice and fat-1 mice fed the n-3 PUFA low CO diet had similar, yet statistically different $\delta^{13} \mathrm{C}$ values. Brain DHA $\delta^{13} \mathrm{C}$ values of wild-type and fat-1 mice fed the CO diet were $-23.12 \pm$ $0.32 \mathrm{mUr}$ and $-21.84 \pm 0.22 \mathrm{mUr}$, respectively. No significant differences were noted in brain DHA $\delta^{13} \mathrm{C}$ values between fat-1 $(-25.17 \pm 0.21 \mathrm{mUr})$ or wild-type mice $(-25.01 \pm 0.25 \mathrm{mUr})$ fed the n-3 PUFA rich COFO diet. This once again suggests that wild-type and fat-1 animals share the same source of brain DHA: dietary preformed n-3 PUFA. These values were significantly less enriched in ${ }^{13} \mathrm{C}$ than brain DHA $\delta^{13} \mathrm{C}$ values of mice fed the $\mathrm{CO}$ diet. 
a)

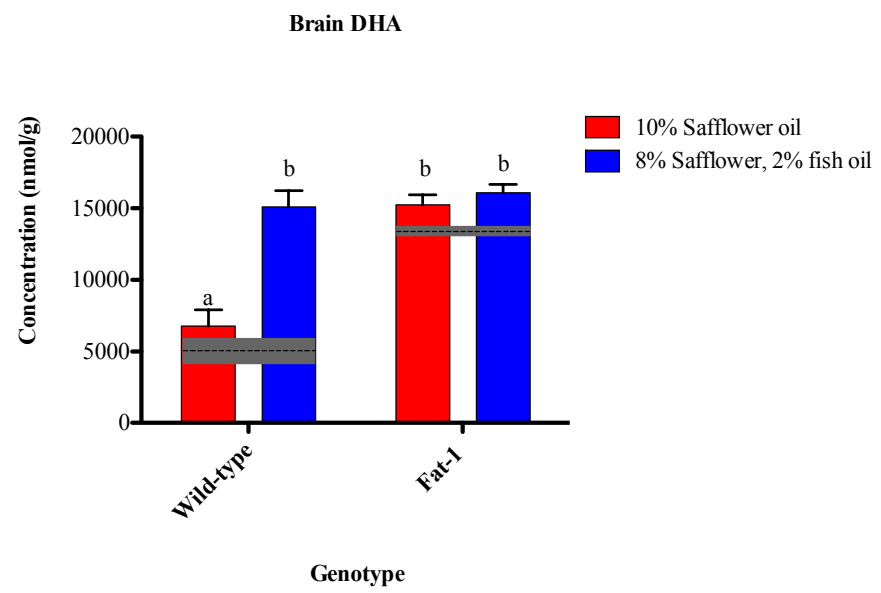

b)

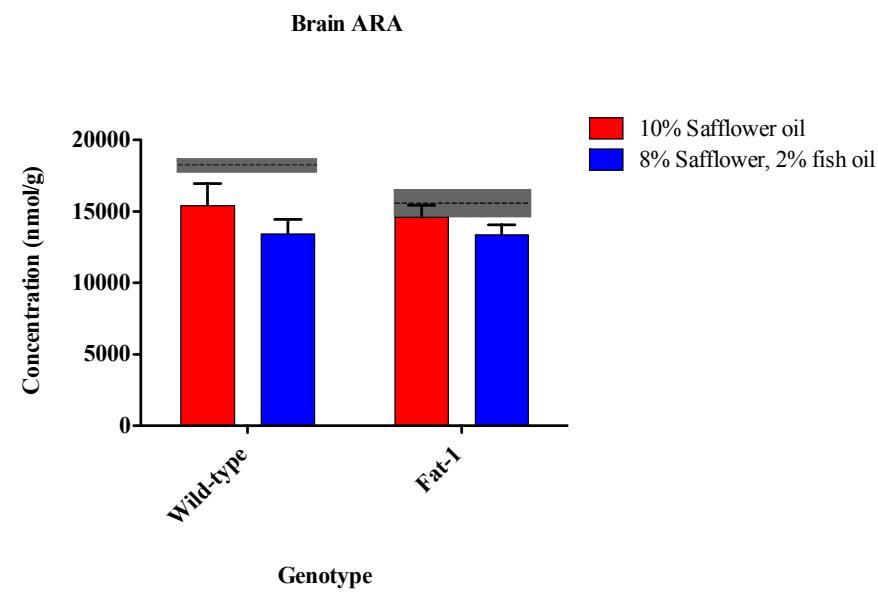

d)
Brain DHA

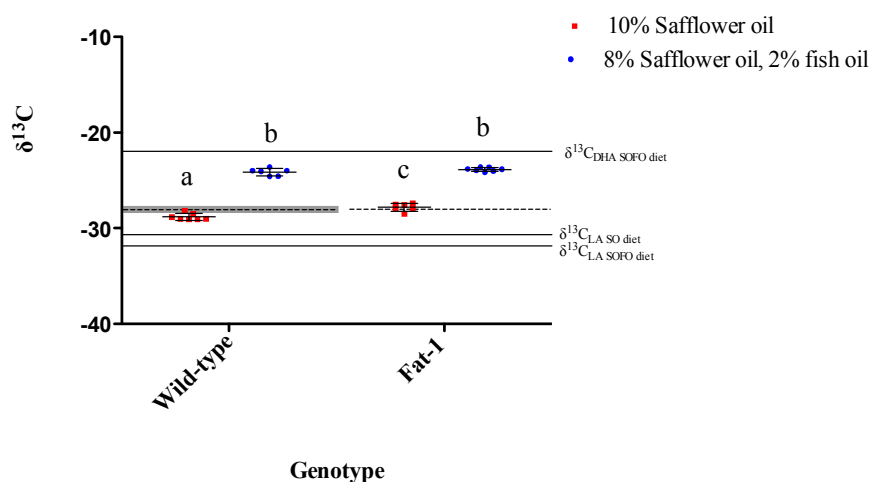

Brain ARA

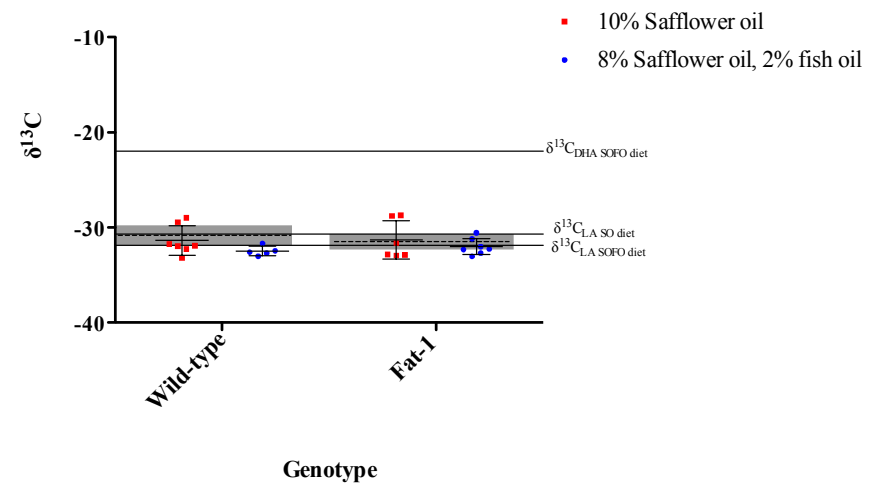

Figure 3.4.1.1: Brain DHA and ARA concentrations and $\delta^{13} \mathrm{C}$ values of animals fed the $10 \%$ safflower oil or $8 \%$ safflower, $2 \%$ fish oil diets

Mean \pm SD of whole brain a) DHA and b) ARA concentrations (nmol/g of brain wet weight) and brain c) DHA and d) ARA $\delta^{13} \mathrm{C}$ values (mUr) of wild-type and fat-1 animals fed the SO or SOFO diet. Dashed lines are indicative of mean baseline values of mice with grey bands representing $\pm \mathrm{SD}$. Solid lines are indicative of dietary fatty acids $\delta^{13} \mathrm{C}$ values. Two-way ANOVA was conducted with Tukey's post hoc test when a significant interaction was found. Different letters reflect significant post hoc differences. There was a significant diet $x$ genotype interaction on brain DHA concentrations $(p<0.0001)$ and a significant diet effect on brain ARA concentrations $(\mathrm{p}<0.01)$. There was a significant diet $\mathrm{x}$ genotype interaction on brain DHA $\delta^{13} \mathrm{C}$ values $(\mathrm{p}=0.01)$ but no main effect or diet $\mathrm{x}$ genotype interaction effect on brain ARA $\delta^{13} \mathrm{C}$ values $(\mathrm{p}>0.05)$. $\mathrm{n}=4-7$ per group. Abbreviations: ARA, arachidonic acid; DHA, docosahexaenoic acid; FO, fish oil; LA, linoleic acid; SO, safflower oil. 
a) Brain DHA

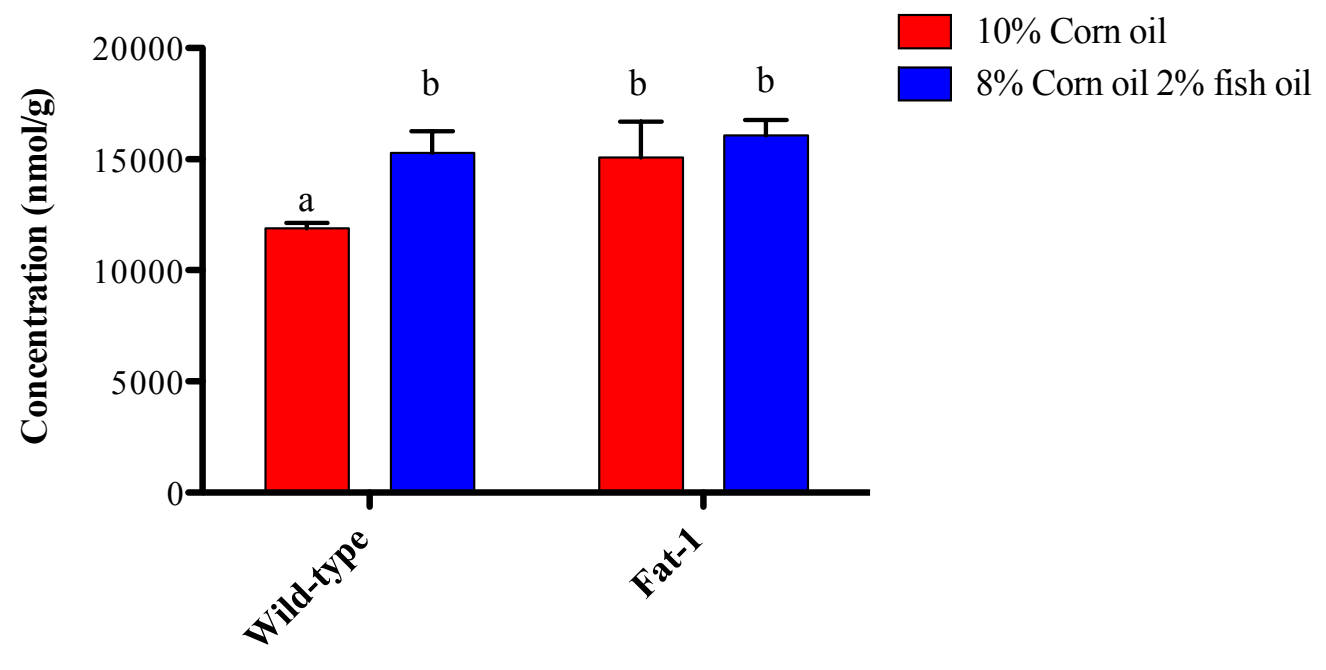

Genotype

b) Brain DHA

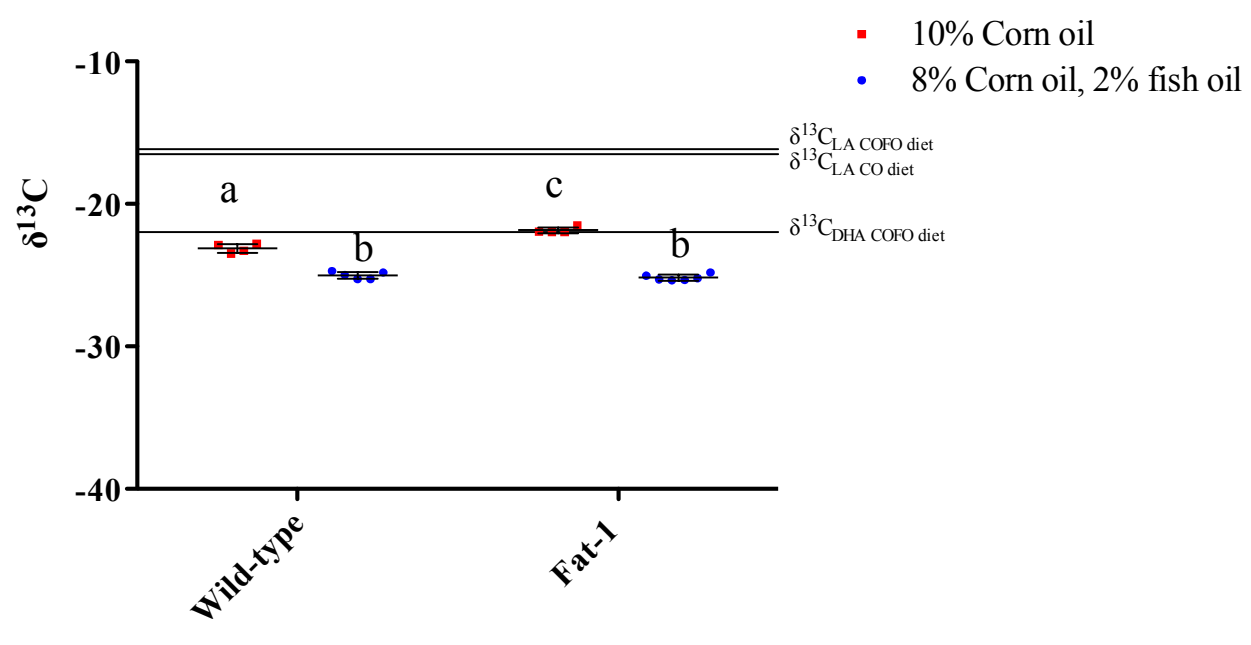

Genotype

Figure 3.4.1.2: Brain DHA concentrations and $\delta^{13} \mathrm{C}$ values of animals fed either the $10 \%$ corn oil diet or $8 \%$ corn oil, $2 \%$ fish oil diet

Mean \pm SD of whole brain DHA a) concentration (nmol/g of brain wet weight) and $\mathbf{b}) \delta^{13} \mathrm{C}$ values (mUr) of wild-type and fat-1 mice weaned onto the $\mathrm{CO}$ and COFO diet. Sold lines are indicative of dietary fatty acid $\delta^{13} \mathrm{C}$ values. A significant interaction effect was detected by two-way ANOVA ( $\mathrm{p}<0.05)$. Tukey's post-hoc test was applied to establish post-hoc differences. Different letters denote significant post-hoc differences. There was a significant diet $\mathrm{x}$ genotype interaction on both brain DHA concentration $(\mathrm{p}<0.05)$ and $\delta^{13} \mathrm{C}$ values ( $\left.\mathrm{p}<0.0001\right) . \mathrm{n}=4-6$. Abbreviations: $\mathrm{CO}$, corn oil; DHA, docosahexaenoic acid; FO, fish oil; LA, linoleic acid. 
n-6 pathway

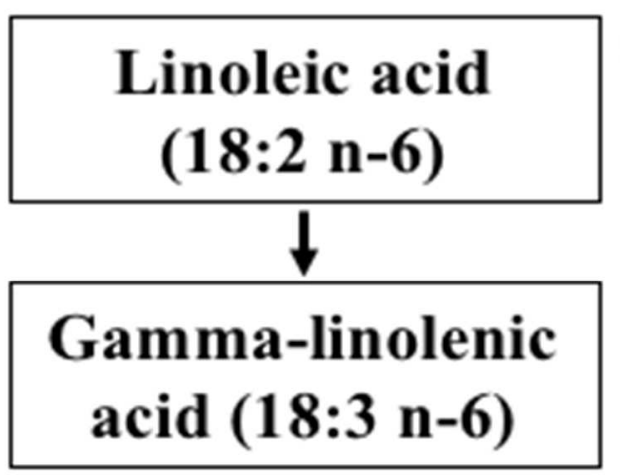

$\downarrow$

Dihomo-gamma

linolenic acid

(20:3 n-6)

$\downarrow$

Arachidonic acid

(20:4 n-6)

$\downarrow$

Adrenic acid

(22:4 n-6)

$\downarrow$

Docosapentaenoic acid

(22:5 n-6) n-3 pathway

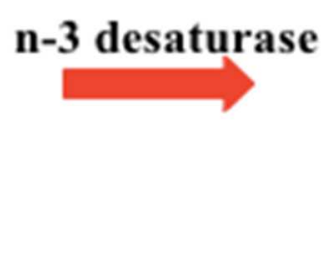

n-3 desaturase

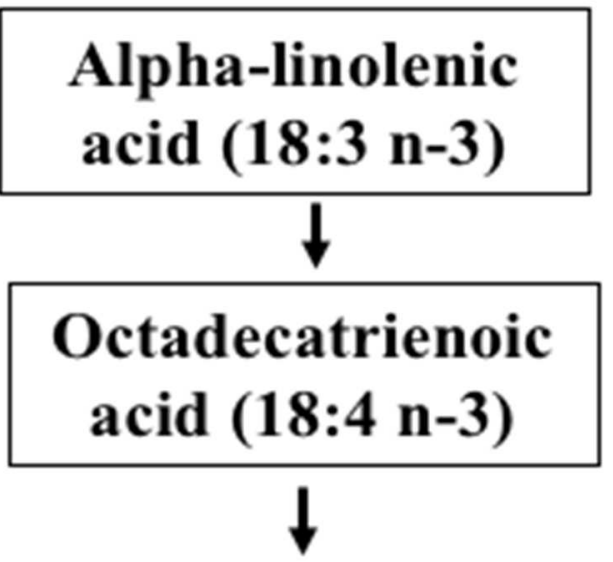

n-3 desaturase

Eicosatetraenoic acid (20:4 n-3)

n-3 desaturase

Eicosapentaenoic acid (20:5 n-3)

$\downarrow$

n-3 desaturase

Docosapentaenoic acid

(22:5 n-3)

$\downarrow$

n-3 desaturase acid (22:6 n-3)

Figure 3.4.2.1: Fat-1 mouse conversion of n-6 PUFA to n-3 PUFA

n-3 and n-6 PUFA synthesis pathway in fat-1 mice. Black arrows represent enzymatic steps present in both fat-1 and wild-type mice. Red arrows represent the theoretical conversion of n-6 PUFA to n-3 PUFA through the activity of the n-3 desaturase enzyme, expressed in fat-1 mice only. 
a)

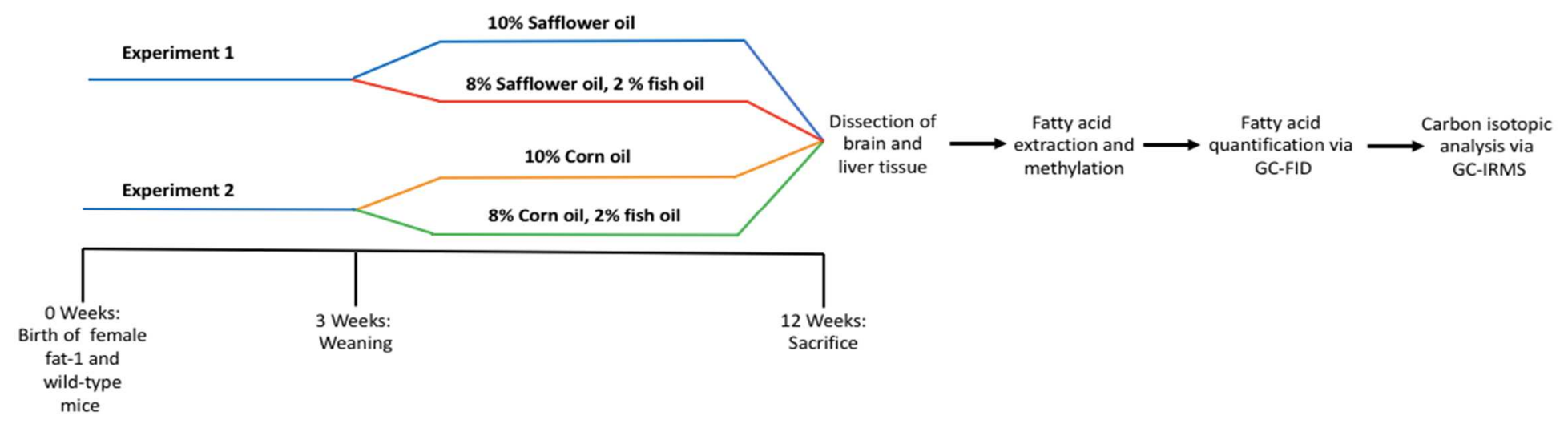

b)

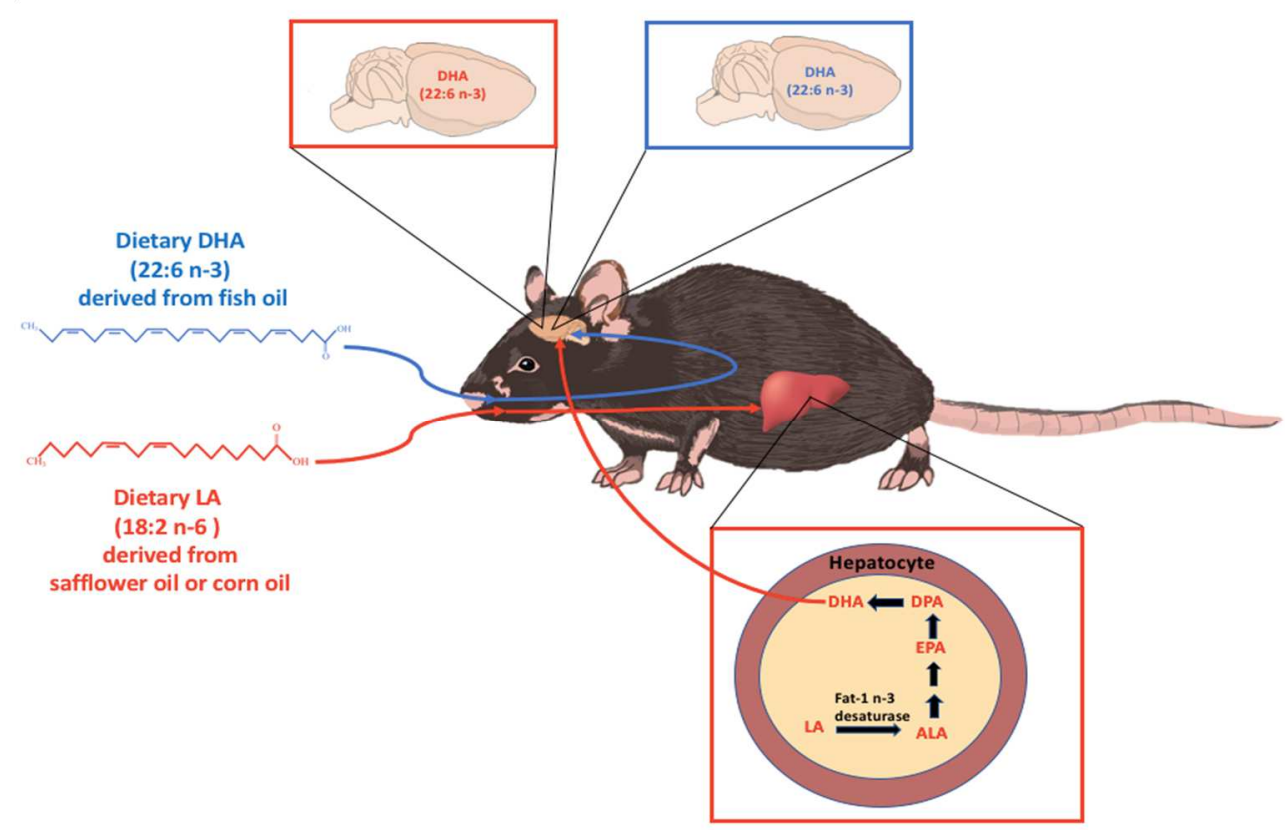

Figure 3.4.2.2: Study design overview and depiction of the hypothetical routes by which fat-1 mice may obtain brain DHA

a) Study design overview. Fat-1 mice and their wild-type littermates were maintained on an n-3 PUFA low, n-6 PUFA rich 10\% safflower oil diet throughout pregnancy and lactation. In the first experiment, mice were either maintained on the n-3 PUFA low, n-6 PUFA rich $10 \%$ safflower diet or weaned on an n-3 and n-6 PUFA rich $8 \%$ safflower, $2 \%$ fish oil diet. Mice were kept on these diets until sacrifice at 12 weeks of age at which point the liver and brain were dissected and prepared for fatty acid quantification by GCFID and carbon isotopic analysis by GC-IRMS. As the carbon isotopic signatures of dietary n-6 PUFA are different than that of the dietary n-3 PUFA in the fish oil diet, the origin of brain DHA can be determined through comparing the isotopic signature of brain DHA to that of these dietary components. To validate our findings, a second experiment was conducted with an identical study design except in this study mice were either weaned on an n-3 PUFA low, n-6 PUFA rich 10\% corn oil diet or an n-3 and n-6 PUFA rich $8 \%$ corn oil, $2 \%$ fish oil diet. In these diets, the main source of $n-6$ PUFA was corn oil, which is more enriched in ${ }^{13} \mathrm{C}$ than n-6 PUFA derived from safflower oil allowing for the validation of our findings. b) Depiction of the hypothetical routes in which fat-1 mice may obtain brain DHA. When fat-1 mice are fed a diet low in n-3 PUFA, they must obtain their brain DHA largely from the synthesis of DHA from dietary n-6 PUFA such as LA (red). However, when fed a diet rich in both n-3 and n-6 PUFA it is unclear if fat-1 mice obtain their brain DHA directly from dietary DHA (blue) or from the synthesis of DHA from n-6 PUFA (red) or a combination of both. As dietary n-3 and n-6 PUFA within our diets are naturally isotopically distinct we were able to determine the dietary origin of brain DHA through the comparison of the carbon isotopic signature of these different dietary components to that of brain DHA.

Abbreviations: ALA, $\alpha$-linolenic acid; DHA, docosahexaenoic acid; DPA, docosapentaenoic acid; EPA, eicosapentaenoic acid; GCFID, gas chromatography-flame ionization detector; GC-IRMS, gas chromatography-isotope ratio mass spectrometry; LA, linoleic acid. 


\subsubsection{Addition of fish oil to the n-6 PUFA rich diets may down regulate synthesis of n-3 PUFA: Mechanistic evidence provided by CSIA by IRMS}

The brain DHA isotopic analysis above suggests that the fat-1 mouse likely obtains its brain DHA from preformed n-3 PUFA rather than synthesis from n-6 PUFA sources. After determining this, we were interested in investigating if this reflected a brain preference for preformed DHA or more simply a lack of hepatic synthesis of DHA from n-6 sources upon consumption of adequate dietary preformed DHA. To do so, we analyzed both liver DHA concentrations and isotopic values. Liver DHA concentrations of mice fed the SO and SOFO diet are presented in (Figure 3.4.3.1 a). As expected, a significant genotype $\mathrm{x}$ diet interaction on liver DHA concentrations was present $(F(1,18)=63.60, p<0.0001)$. Liver DHA concentration was significantly lower in wild-type mice fed the SO diet $(n=5)$ in comparison to wild-type mice fed the SOFO diet $(\mathrm{n}=6)$ and fat- 1 mice fed the SO $(\mathrm{n}=6)$ or SOFO diet $(\mathrm{n}=5)$. Wild-type and fat-1 mice fed the SOFO diet also had significantly higher liver DHA concentrations in comparison to fat-1 mice fed the SO diet but did not differ from one another. The liver DHA concentrations of animals fed the $\mathrm{CO}$ and $\mathrm{COFO}$ are displayed in Figure 3.4.3.2 b. Consistent with the previous results, there was a significant genotype $\mathrm{x}$ diet interaction effect on liver DHA concentrations $(F(1,13)=10.97, p<0.01)$. Wild-type mice fed the low $n-3$ PUFA CO diet $(n=4)$ had significantly lower DHA levels than fat-1 mice fed the $\mathrm{CO}(\mathrm{n}=4)$ or COFO diet $(\mathrm{n}=4)$, or wild-type mice fed the COFO diet $(n=4)$.

Isotopic analysis of liver DHA of animals consuming the SO and SOFO diets are presented in Figure 3.4.3.1 b. Wild-type mice fed the SO diet did not have DHA concentrations high enough to obtain a valid $\delta^{13} \mathrm{C}$ value and therefore were not included in the analysis. Similar to the brain isotopic analysis, fat-1 mice fed the SO diet had a significantly more ${ }^{13} \mathrm{C}$ deplete $\delta^{13} \mathrm{C}$ value ($29.92 \pm 1.02 \mathrm{mUr}$ ) than liver DHA $\delta^{13} \mathrm{C}$ values of the wild-type and fat- 1 mice fed the SOFO diet $(F(2,14)=78.04, p<0.0001)$. These animals had a liver DHA $\delta^{13} \mathrm{C}$ of $-24.50 \pm 0.63 \mathrm{mUr}$ and $23.65 \pm 1.08 \mathrm{mUr}$, respectively and did not differ statistically.

Carbon isotopic analysis of liver DHA of animals fed the $\mathrm{CO}$ and $\mathrm{COFO}$ diets are displayed in

Figure 3.4.3.1 b. Although there was not a significant genotype $x$ diet interaction effect or main effect of genotype $(F(1,12)=0.04, p>0.05)$, there was a significant main effect of diet on carbon 
isotopic signature $(\mathrm{F}(1,12)=328.2, \mathrm{p}<0.0001)$. Wild-type and fat- 1 mice fed the COFO diet had a liver DHA $\delta^{13} \mathrm{C}$ of $-25.28 \pm 0.6 \mathrm{mUr}$ and $-25.31 \pm 0.97 \mathrm{mUr}$ while wild-type and fat-1 mice fed the $\mathrm{CO}$ diet displayed $\delta^{13} \mathrm{C}$ values of $-19.35 \pm 0.48 \mathrm{mUr}$ and $-19.23 \pm 0.47 \mathrm{mUr}$, respectively. The lack of difference in brain and liver $\delta^{13} \mathrm{C}$ values between fat- 1 and wild-type mice fed either the SOFO or COFO diet suggests that in the presence of preformed DHA, hepatic synthesis of DHA from n-6 PUFA in fat-1 mice is downregulated. 
a)

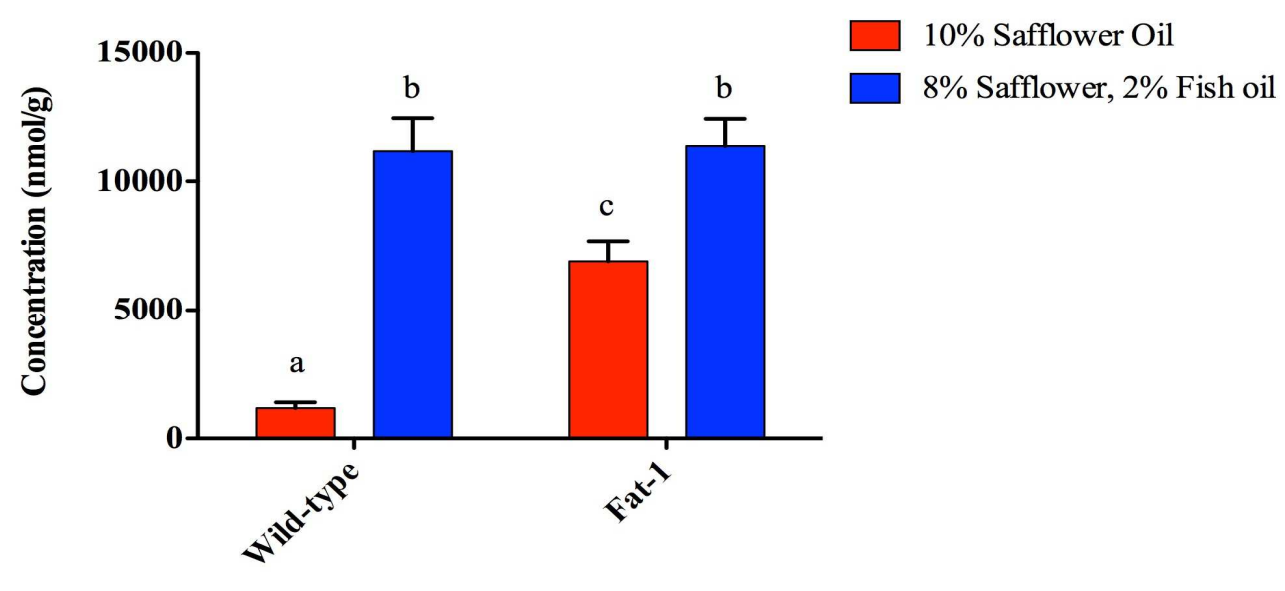

Genotype

Liver DHA

b)

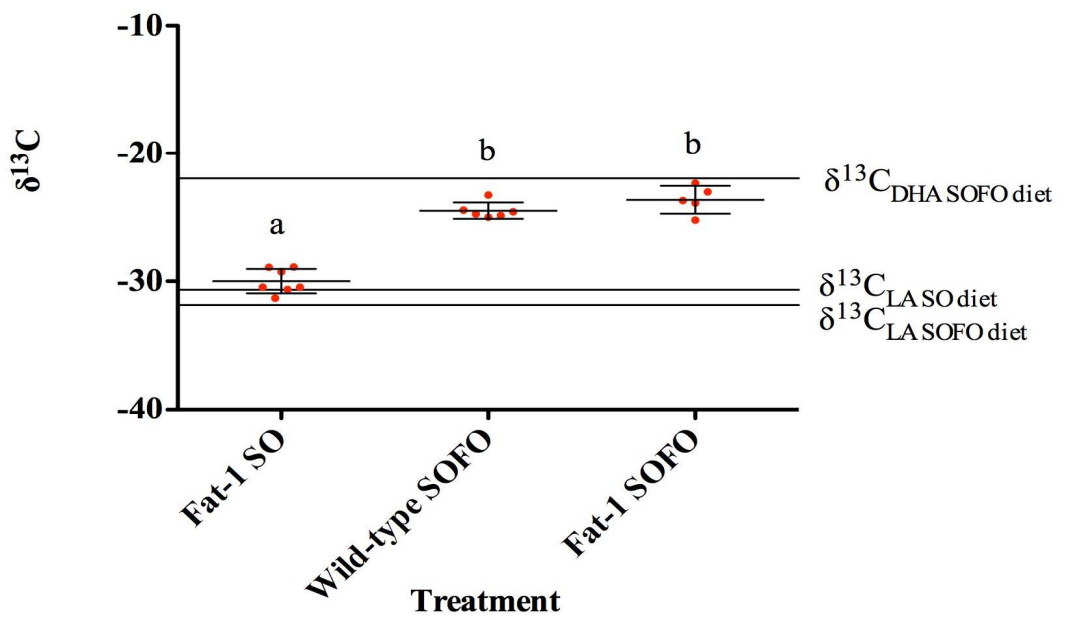

Figure 3.4.3.1: Liver DHA concentrations and $\delta^{13} \mathrm{C}$ values of animals fed either the $10 \%$ safflower oil diet or $8 \%$ safflower oil, $2 \%$ fish oil diet

Mean \pm SD of liver DHA a) concentration (nmol/g of liver wet weight) and b) $\delta^{13} \mathrm{C}$ values (mUr) of wild-type and fat-1 mice weaned onto the SO and SOFO diet. Sold lines are indicative of dietary fatty acid $\delta^{13} \mathrm{C}$ values. Wild-type animals fed the SO diet had liver DHA concentrations that were too low for reliable detection of $\delta^{13} \mathrm{C}$ values and were therefore excluded from this analysis. Liver DHA concentrations were analyzed via two-way ANOVA with Tukey's post-hoc test upon detection of a significant diet $\mathrm{x}$ genotype interaction. Liver DHA $\delta^{13} \mathrm{C}$ values were analyzed via One-way ANOVA. Different letters denote significant post-hoc differences. There was a significant genotype $x$ diet interaction on liver DHA concentration $(p<0.0001)$ and $\delta^{13} \mathrm{C}$ values $(\mathrm{p}<0.0001) . \mathrm{n}=5-6$ per group. Abbreviations: DHA, docosahexaenoic acid; FO, fish oil; LA, linoleic acid; SO, safflower oil. 


\section{Liver DHA}

a)

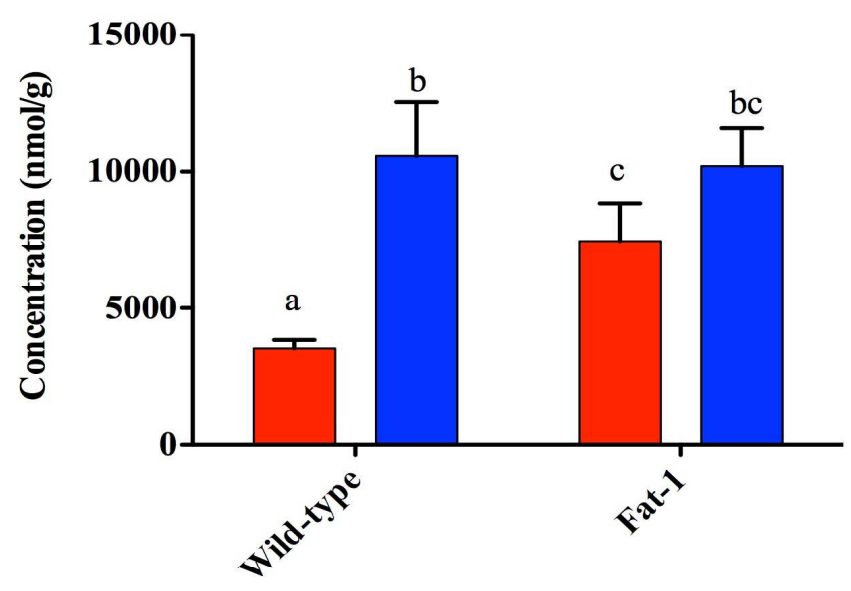

$10 \%$ Corn Oil

$8 \%$ Corn Oil, $2 \%$ Fish oil

Genotype

Liver DHA

b)

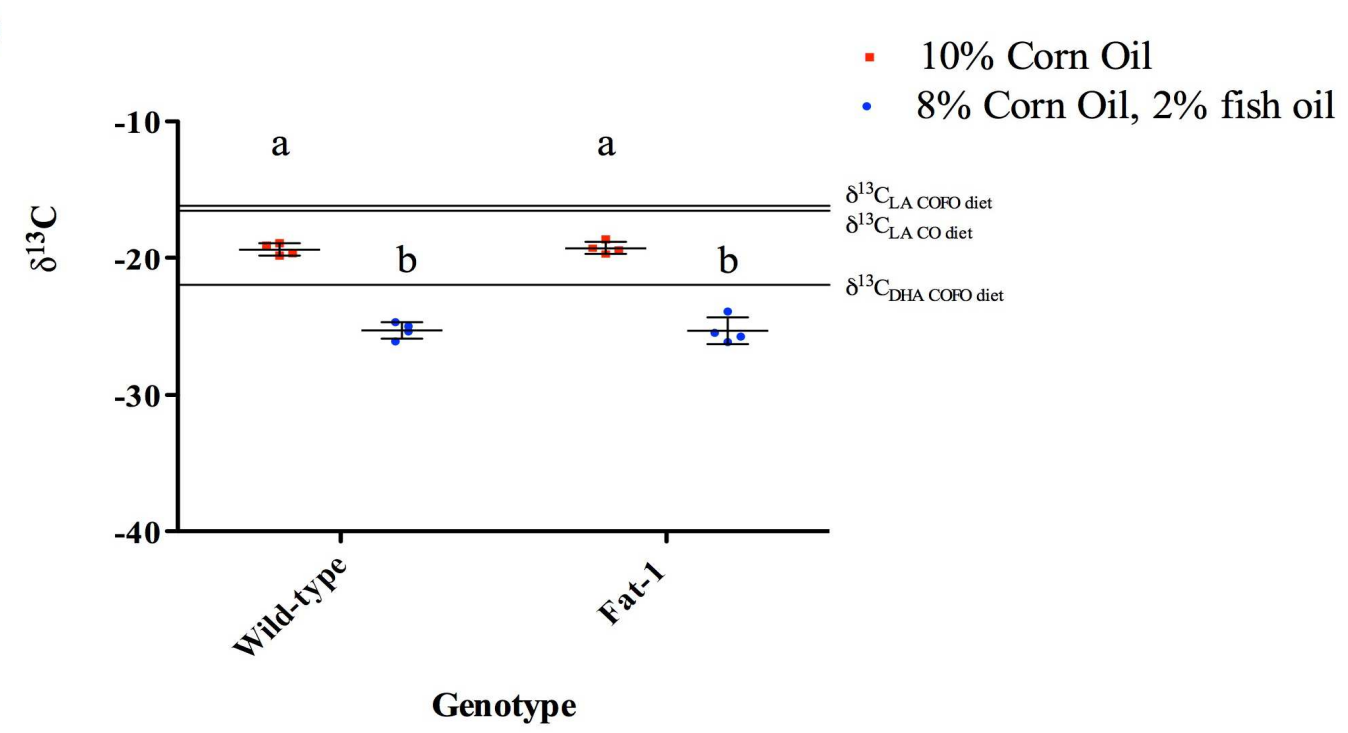

Figure 3.4.3.2 Liver DHA concentrations and $\delta^{13} \mathrm{C}$ values of animals fed either the $10 \%$ corn oil or $8 \%$ corn oil, $2 \%$ fish oil diets

Mean \pm SD of liver DHA a) concentration (nmol/g of liver wet weight) and b) $\delta^{13} \mathrm{C}$ values (mUr) of wild-type and fat-1 mice weaned onto the $\mathrm{CO}$ and COFO diet. Sold lines are indicative of dietary fatty acid $\delta^{13} \mathrm{C}$ values. Statistical analysis was conducted via two-way ANOVA along with Tukey's post-hoc test when a significant genotype $\mathrm{x}$ diet interaction was detected. Different letters are indicative of significant post-hoc differences. There was a significant genotype $\mathrm{x}$ diet interaction on liver DHA concentration $(\mathrm{p}=0.01)$. There was a significant main effect of diet on liver $\delta^{13} \mathrm{C}$ values $(\mathrm{p}<0.0001) . \mathrm{n}=4$ per group. Abbreviations: $\mathrm{CO}$, corn oil; DHA, docosahexaenoic acid; FO, fish oil; LA, linoleic acid. 


\subsection{Discussion}

Previously, our group assessed if the addition of n-3 PUFA to an n-6 PUFA rich diet could increase brain DHA concentrations in fat-1 mice beyond the levels achieved by endogenous synthesis from n-6 PUFA [88]. Interestingly, no difference in either total brain DHA or DHA esterified to phospholipids was noted regardless of if the fat- 1 animals were fed a $10 \%$ safflower oil diet or an $8 \%$ safflower oil $2 \%$ fish oil diet. Although these animals achieve similar brain DHA levels, it remained unclear if fat-1 mice fed the fish-oil containing diet preferentially obtained their brain DHA via synthesis from n-6 PUFA or through the uptake of dietary preformed DHA. A key factor that limited our group from resolving this question was the lack of reliable, cost-efficient methodologies available to study the origin of brain fatty acids. Here, we demonstrate the power of CSIA by IRMS to successfully trace the origin of brain DHA in this complex model. Through this approach, we have identified dietary n-3 PUFA as the main contributor to brain DHA in fat-1 mice fed diets rich in both n-3 and n-6 PUFA. We further demonstrated the remarkable potential of this method to study biochemical pathways through providing evidence that fish oil may downregulate hepatic synthesis of n-3 PUFA from n-6 PUFA sources. To our knowledge, this study reflects the first application of CSIA in a complex multivariate transgenic model to resolve a question pertaining to the origin of tissue fatty acids.

In the present analysis, fat- 1 and wild-type mice were fed fish-oil containing diets rich in $\mathrm{n}-3$ and n-6 PUFA. The main n-3 and n-6 PUFA contained in these diets displayed isotopically distinct values. Despite this, the brain DHA $\delta^{13} \mathrm{C}$ values between fat- 1 and wild-type mice fed the SOFO diet or the COFO diets were indistinguishable. These results largely support that in the presence of preformed n-3 PUFA and excess n-6 PUFA, fat-1 mice, like wild-type mice, obtain their brain DHA solely from dietary n-3 PUFA sources and not via synthesis from n-6 PUFA. In line with this evidence, we also found that liver DHA $\delta^{13} \mathrm{C}$ values between wild-type and fat-1 mice fed either of the fish-oil containing diets also did not differ. Taken together, this demonstrates that in the presence of adequate preformed DHA, fat-1 mice down regulate synthesis of DHA from n-6 PUFA sources. LA was largely the most abundant n-6 PUFA present in each diet and therefore likely the main substrate acted upon by the fat-1 n-3 desaturase enzyme. If acted upon by this enzyme, dietary LA would undergo desaturation to produce ALA, which could then act as a 
precursor in the synthesis of DHA. However, multiple studies have suggested that in the presence of preformed DHA, biosynthesis of DHA from ALA is not a large contributor to brain DHA concentrations. In one 20-day feeding study [37], 8-day old rat pups were fed diets in which all dietary ALA was isotopically labeled. They noted that in the presence of preformed DHA, isotopically labeled DHA derived from ALA synthesis markedly declined in both the brain and liver [37]. Studies utilizing a five-minute infusion model have also supported that in rats fed a diet rich in ALA and DHA, DHA biosynthesized from ALA is not a significant contributor to brain DHA concentrations [25,101]. Furthermore, in the presence of dietary preformed DHA delta-5 and delta-6 desaturase enzymes involved in the synthesis of DHA from ALA decline in expression and activity [102-104]. Considering this, it is possible that fat-1 mice fed the SOFO and COFO diet obtained their brain DHA solely from dietary n-3 PUFA because the presence of dietary preformed DHA downregulates sufficient biosynthesis of DHA from ALA produced through LA desaturation.

Although dietary n-3 PUFA was determined to be the main source of brain DHA in mice fed the FO diets, brain DHA $\delta^{13} \mathrm{C}$ values of these animals were less enriched in ${ }^{13} \mathrm{C}$ than the DHA present in these diets. The brain DHA $\delta^{13} \mathrm{C}$ values of mice the FO diets differed by approximately $2 \mathrm{mUr}$. It is key to note that our experimental animals were introduced to these diets at three weeks of age for a period of 9 weeks. It is therefore likely that this difference in enrichment is attributable to the lack of complete turn-over of brain DHA acquired in utero and through lactation prior to weaning. Indeed, the half-life of DHA in brain phospholipids has been reported to be 33 days in rat-pups fed an n-3 PUFA adequate diet [105]. If this half-life were extrapolated to mice, it is likely that mice weaned onto the SOFO and COFO diets did not turn over approximately $25 \%$ of brain DHA acquired prior to weaning. Further, the SOFO and COFO diets also contained EPA, an n-3 PUFA precursor for the synthesis of DHA (2.4\% and $2.5 \%$ of total fatty acids, respectively). As EPA present in each diet was less enriched in ${ }^{13} \mathrm{C}$ than dietary DHA, it is possible that DHA derived from this source may have also added to brain DHA concentrations and contributed to the ${ }^{13} \mathrm{C}$ depletion noted.

The precision and cost-efficiency provided by CSIA by IRMS are key assets of this tool. As mentioned, methods typically applied to trace the origin of brain lipids involve the synthesis and administration of expensive, fatty acid tracers. These tracers are normally detected in samples 
using conventional mass spectrometers, which are capable of detecting ${ }^{13} \mathrm{C}$ enrichment levels of 0.5 atom percent excess (APE) and greater[44]. At the natural abundance level most variations in ${ }^{13} \mathrm{C}$ enrichment are within the range of 1.0343-1.1167 APE[42], while in the present analysis the largest difference between analyzed samples was 0.019 APE. This level of enrichment is therefore much too low to be detected by conventional mass spectrometry. However, unlike traditional mass spectrometers, an IRMS is equipped with multiple detectors, each scanning specifically for only one carbon dioxide isotopomer. This allows for recognition of ${ }^{13} \mathrm{C}$ enrichment of $\sim 0.0003$ APE, a level of precision capable of identifying minute variations in ${ }^{13} \mathrm{C}$ enrichment at the natural abundance level[44]. In the present analysis, we took advantage of this remarkable precision to bypass the need to purchase costly labelled fatty acid tracers. Through feeding experimental animals diets that were isotopically distinguished at the natural abundance level, CSIA allowed for the chronic tracing of fatty acids in vivo. While diets purchased for this study did cost approximately five thousand dollars, we estimate that replacing all n-3 and n-6 PUFA within our diets with labelled tracers would have cost about 2000 times more, or upwards of ten million dollars. This value was approximated using the retail value of n-3 and n-6 PUFA tracers while taking into account our study duration and the amount of n-3 and n-6 PUFA within the utilized diets. Overcoming this cost barrier provides a means for future chronic feeding studies to be conducted in order to solve crucial questions pertaining to brain lipid metabolism that would have been previously infeasible to answer.

In the present analysis, we demonstrated the potential use of CSIA by IRMS to aid in understanding complex biochemical pathways. Through isotopically analyzing liver tissue we determined that hepatic synthesis of n-3 PUFA from n-6 PUFA is not a major contributor to liver DHA concentrations in fat-1 mice fed fish oil diets, suggesting that DHA synthesis may be downregulated in this group. CSIA by IRMS has also been applied sparingly in other physiologically relevant cases as an extremely cost-efficient means to assess the chronic metabolism of tissue fatty acids. Through switching individuals consuming certain diets onto diets that are isotopically distinct, it is possible to apply CSIA by IRMS to study fatty acid turn over and kinetics. This approach has been applied previously in order to estimate synthesis rates of ARA[66,67] and DHA[67] from plasma in infants. Further, through the use of mass balance and pool mixing equations, it is possible to use CSIA by IRMS to estimate the contribution of different dietary sources to tissue fatty acids[95,106]. Indeed, Hixson et al (2014) applied this 
idea to estimate the contribution of dietary ALA derived from camelina oil to total DHA concentrations in rainbow trout[107]. Work from our laboratory demonstrated the use of this approach to estimate the contribution of DHA retroconversion to liver EPA concentrations in rats fed a DHA rich diet[69]. Recently, our group also utilized a two-end member mixing model with CSIA by IRMS to understand the contribution of dietary palmitic acid and lipogenesis to brain palmitic acid levels (Unpublished results). These studies largely support the use of CSIA by IRMS as a cost-efficient alternative to current methods applied to study metabolism, which can involve the administration of fatty acid tracers. This approach may be especially useful as an ethical substitute to the administration of radioactive tracers which tend to raise safety concerns.

Our present work demonstrates the ability of CSIA by IRMS to detect minute differences in ${ }^{13} \mathrm{C}$ enrichment. As mentioned, an IRMS can measure ${ }^{13} \mathrm{C}$ enrichment with the precision of $\sim 0.0003$ APE[44]. In the present study, fat-1 and wild-type mice fed the same SO diet obtained brain DHA $\delta^{13} \mathrm{C}$ values that displayed a mean difference of $0.99 \mathrm{mUr}(\sim 0.001 \mathrm{APE})$. This is in line with previous work conducted in our lab in which CSIA successfully resolved a $0.78 \mathrm{mUr}$ difference in brain DHA $\delta^{13} \mathrm{C}$ values between $\mathrm{F} 1$ and $\mathrm{F} 2$ progeny of mice fed the same $\operatorname{diet}[70]$. These results largely support the potential application of this method to discriminate between slight differences in metabolic pathways or small differences in brain fatty acid turnover.

To conclude, our study has successfully demonstrated, for the first time, the application of CSIA to determine the origin of brain DHA in a multivariate model: the fat- 1 mouse. Future analyses should continue to apply this powerful methodology to complex, multivariate models to further understand the origin and metabolism of tissue fatty acids and their metabolism. 


\section{Chapter 4 \\ General discussion and conclusions}




\subsection{Limitations}

Several factors limit the conclusions made by the present analysis. To begin with, the diets utilized in the presented experiments contained dietary fish oil as a source of preformed DHA. Fish oil does, however, contain 2.5\% EPA and trace amounts of DPA n-3 which could be used for the synthesis of DHA. The present analysis, therefore, cannot rule out the contribution of these fatty acids to brain DHA concentrations. Consequently, our findings can conclude that brain DHA is derived from dietary fish oil in fat-1 mice and not solely preformed dietary DHA. Furthermore, within the diets utilized, the only n-6 PUFA present abundantly was LA. Although DHA derived from LA does not appear to be a significant contributor to brain DHA concentrations in fish oil fed fat- 1 mice, we cannot assume the same would be true if a different n-6 PUFA such as ARA were abundantly present instead. Finally, ALA was present in small quantities in both the $10 \%$ safflower oil and $10 \%$ corn oil diets $(0.3 \%$ and $0.9 \%$, respectively). As a result, it was not possible to obtain a valid isotopic value for these dietary fatty acids. This limits our ability to estimate the contribution of dietary ALA to brain DHA concentrations. This is especially limiting when assessing wild-type animals consuming these diets as ALA is the sole n-3 PUFA present within their diet.

In the present study, liver DHA of fat- 1 and wild-type animals fed fish-oil containing diets were not isotopically distinct. This led to the conclusion that hepatic synthesis of n-3 PUFA from n-6 PUFA in fat-1 mice was not a large contributor to liver DHA concentration, and therefore may be down-regulated. However, the present work did not analyze hepatic enzyme activity or gene expression. To confirm this conclusion, future work should assess if enzyme activity or expression are downregulated upon fish oil feeding, and if so which specific enzymes are affected

Although CSIA by IRMS has proven to be a powerful, cost-efficient tool for studying the routing and metabolism of brain fatty acids, it is not without limitations. Although gaining popularity, an isotope ratio mass spectrometer is costly and is not commonly used in the field of lipid chemistry. As such, the availability of this instrument to perform CSIA may be limited. Further, preparing non-volatile compounds such as fatty-acids for GC-IRMS does require derivatization which may introduce slight error. Although we have corrected for this in our current analysis, it is still possible that the use of EA-IRMS to obtain correction values could introduce a slight 
systematic error as GC-IRMS was utilized to analyze our prepared samples. Finally, obstacles may be encountered when the concentration of the analyte of interest is below the limit of detection to reliably obtain a $\delta^{13} \mathrm{C}$ value, as was the case for dietary ALA in the SO diet $(0.3 \%$ total fatty acids).

\subsection{Future directions}

The present analysis has provided the proof-of-concept for the use of CSIA by IRMS to study the origin of brain fatty acids. Future work could combine this approach with mathematical modeling[95] to study the metabolism and turnover of tissue fatty acids. It is crucial to note that in order to utilize CSIA by IRMS to study metabolism, tissue isotopic values must be equilibrated to their experimental diets. After equilibrium is achieved, animals can then be switched onto an isotopically distinct diet and tissue fatty acid metabolism can be assessed using two-end member mixing models. Recent work from our group has demonstrated that through maintaining mice on a diet over two generations, it is possible to equilibrate brain fatty acid $\delta^{13} \mathrm{C}$ values to that of their dietary origin[70]. Future work could equilibrate brain isotopic values of animals over multiple generations and apply diet-switching techniques in order to study the turnover of brain fatty acids in animal models. Further, this approach should be applied to analyze how changing dietary fatty acid levels and composition may alter brain fatty acid turn over.

Future work should focus on validating the use of natural abundance CSIA by IRMS as a tool for biomarker detection. Indeed, previous studies have supported that blood compound isotopic analysis can reflect dietary intake. Choy et al (2013) have demonstrated that $\delta^{13} \mathrm{C}$ values of alanine in red blood cells can correlate strongly to sugar sweetened beverage consumption[108]. Rhee et al (1997) have also shown that upon consumption of a controlled diet for 5 weeks, human serum fatty acid $\delta^{13} \mathrm{C}$ values are markedly altered and reflective of their provided $\operatorname{diet}[68]$. Recently, our group also provided evidence that mouse serum and red blood cell $\delta^{13} \mathrm{C}$ values largely resemble the $\delta^{13} \mathrm{C}$ values of their dietary fatty acids[70]. As self-report measures of dietary intake can introduce error and recall bias, validation of these reports through the detection of reliable biomarkers is crucial. CSIA by IRMS may provide a means to aid in this validation when the anylate of interest is isotopically distinct from other dietary components. It 
may further provide an opportunity to test for compliance in randomize controlled trials where adherence to supplements or a specific dietary regime is crucial.

\subsection{Significance}

The work presented in this thesis has provided the proof-of-concept for the use of natural abundance CSIA by IRMS to trace the dietary origins of tissue fatty acids. This provides a costefficient means to study the origin and metabolism of tissue fatty acids through chronic feeding, a task that was previously infeasible to execute. As this method essentially utilizes food as tracers, it may be particularly useful in future studies as an ethical approach to studying fatty acid metabolism in humans.

The work presented in this thesis has also, for the first time, discovered that fat- 1 mice fed fish oil containing diets obtain brain DHA predominately from preformed dietary n-3 PUFA and not from synthesis from n-6 PUFA. We further demonstrated that upon fish oil feeding, hepatic synthesis of n-3 PUFA from n-6 PUFA may be downregulated. This data reflects that synthesis of n-3 PUFA from n-6 PUFA in the fat- 1 mouse model is largely driven by n-3 PUFA deficiency.

\subsection{Conclusion}

In the provided work we were able to establish that:

1) Fat-1 mice fed a fish oil containing diet obtain their brain DHA predominantly from preformed n-3 PUFA and not from the synthesis of n-3 PUFA from n-6 PUFA.

2) Natural abundance CSIA by IRMS is a valid, cost-efficient method to trace the dietary origin of tissue fatty acids. 


\section{Chapter 5: References}

[1] Biochemical, Physiological, and Molecular Aspects of Human Nutrition, 3rd Edition 9781437709599.

[2] J.J. Liu, P. Green, J. John Mann, S.I. Rapoport, M.E. Sublette, Pathways of polyunsaturated fatty acid utilization: Implications for brain function in neuropsychiatric health and disease, Brain Res. 1597 (2015) 220-246. doi:10.1016/j.brainres.2014.11.059.

[3] A.C. Patterson, K.D. Stark, Direct Determinations of the Fatty Acid Composition of Daily Dietary Intakes Incorporating Nutraceuticals and Functional Food Strategies to Increase n3 Highly Unsaturated Fatty Acids.

[4] S.C. Cunnane, M.J. Anderson, The majority of dietary linoleate in growing rats is betaoxidized or stored in visceral fat., J. Nutr. 127 (1997) 146-152.

[5] A.F. Domenichiello, A.P. Kitson, R.P. Bazinet, Is docosahexaenoic acid synthesis from $\alpha$ linolenic acid sufficient to supply the adult brain?, Prog. Lipid Res. 59 (2015) 54-66. doi:10.1016/j.plipres.2015.04.002.

[6] A.F. Domenichiello, C.T. Chen, M.-O. Trepanier, P.M. Stavro, R.P. Bazinet, Whole body synthesis rates of DHA from $\alpha$-linolenic acid are greater than brain DHA accretion and uptake rates in adult rats., J. Lipid Res. 55 (2014) 62-74. doi:10.1194/jlr.M042275.

[7] B.J. Meyer, N.J. Mann, J.L. Lewis, G.C. Milligan, A.J. Sinclair, P.R.C. Howe, Dietary intakes and food sources of omega- 6 and omega-3 polyunsaturated fatty acids, Lipids. 38 (2003) 391-398. doi:10.1007/s11745-003-1074-0.

[8] T.A. Hagve, B.O. Christophersen, Effect of dietary fats on arachidonic acid and eicosapentaenoic acid biosynthesis and conversion to $\mathrm{C} 22$ fatty acids in isolated rat liver cells., Biochim. Biophys. Acta. 796 (1984) 205-17.

[9] E.A. Emken, R.O. Adlof, R.M. Gulley, Dietary linoleic acid influences desaturation and acylation of deuterium-labeled linoleic and linolenic acids in young adult males., Biochim. Biophys. Acta. 1213 (1994) 277-88.

[10] S.M. Innis, Dietary (n-3) fatty acids and brain development., J. Nutr. 137 (2007) 855-9.

[11] F. Bosetti, Arachidonic acid metabolism in brain physiology and pathology: lessons from genetically altered mouse models., J. Neurochem. 102 (2007) 577-86. doi:10.1111/j.14714159.2007.04558.x.

[12] R.P. Bazinet, S. Layé, Polyunsaturated fatty acids and their metabolites in brain function and disease, Nat. Rev. Neurosci. 15 (2014) 771-785. doi:10.1038/nrn3820.

[13] J. Alessandri, P. Guesnet, S. Vancassel, P. Astrog, I. Denis, B. Langelier, S. Aid, C. 
Poumes-Ballihaut, G. Champeil-Potokar, M. Lavialle, Polyunsaturated fatty acids in the central nervous system: evolution of concepts and nutritional implications throughout life, Reprod. Nutr. Dev. 44 (2004) 509-538. doi:10.1051/rnd:2004063.

[14] E.E. Birch, D.R. Hoffman, R. Uauy, D.G. Birch, C. Prestidge, Visual Acuity and the Essentiality of Docosahexaenoic Acid and Arachidonic Acid in the Diet of Term Infants, Pediatr. Res. 44 (1998) 201-209. doi:10.1203/00006450-199808000-00011.

[15] K. Davis-Bruno, M.S. Tassinari, Essential fatty acid supplementation of DHA and ARA and effects on neurodevelopment across animal species: a review of the literature, Birth Defects Res B Dev Reprod Toxicol. 92 (2011) 240-250. doi:10.1002/bdrb.20311.

[16] R.K. McNamara, Role of Omega-3 fatty acids in the etiology, treatment, and prevention of depression: Current status and future directions, J. Nutr. Intermed. Metab. 5 (2016) 96106. doi:10.1016/J.JNIM.2016.04.004.

[17] Y. Zhang, J. Chen, J. Qiu, Y. Li, J. Wang, J. Jiao, Intakes of fish and polyunsaturated fatty acids and mild-to-severe cognitive impairment risks: a dose-response meta-analysis of 21 cohort studies, Am. J. Clin. Nutr. 103 (2016) 330-340. doi:10.3945/ajcn.115.124081.

[18] S.R. De Vriese, A.B. Christophe, M. Maes, Lowered serum n-3 polyunsaturated fatty acid (PUFA) levels predict the occurrence of postpartum depression: further evidence that lowered n-PUFAs are related to major depression., Life Sci. 73 (2003) 3181-7.

[19] M. Maes, R. Smith, A. Christophe, P. Cosyns, R. Desnyder, H. Meltzer, Fatty acid composition in major depression: decreased omega 3 fractions in cholesteryl esters and increased C20: 4 omega 6/C20:5 omega 3 ratio in cholesteryl esters and phospholipids., J. Affect. Disord. 38 (1996) 35-46. doi:10.1016/0165-0327(95)00092-5.

[20] P.B. Adams, S. Lawson, A. Sanigorski, A.J. Sinclair, Arachidonic acid to eicosapentaenoic acid ratio in blood correlates positively with clinical symptoms of depression., Lipids. 31 Suppl (1996) S157-61.

[21] B. Heude, P. Ducimetière, C. Berr, EVA Study, Cognitive decline and fatty acid composition of erythrocyte membranes - The EVA Study, Am. J. Clin. Nutr. 77 (2003) 803-808. doi:10.1093/ajcn/77.4.803.

[22] A.A. Farooqui, L.A. Horrocks, T. Farooqui, Modulation of inflammation in brain: a matter of fat, J. Neurochem. 101 (2006) 577-599. doi:10.1111/j.1471-4159.2006.04371.x.

[23] F. Bosetti, Arachidonic acid metabolism in brain physiology and pathology: lessons from genetically altered mouse models, J. Neurochem. 102 (2007) 577-586. doi:10.1111/j.1471-4159.2007.04558.x.

[24] C.N. Serhan, Pro-resolving lipid mediators are leads for resolution physiology., Nature. 510 (2014) 92-101. doi:10.1038/nature13479.

[25] J.C. DeMar, K. Ma, L. Chang, J.M. Bell, S.I. Rapoport, $\alpha$-linolenic acid does not contribute appreciably to docosahexaenoic acid within brain phospholipids of adult rats 
fed a diet enriched in docosahexaenoic acid, J. Neurochem. 94 (2005) 1063-1076. doi:10.1111/j.1471-4159.2005.03258.x.

[26] J.C. DeMar, H.J. Lee, K. Ma, L. Chang, J.M. Bell, S.I. Rapoport, R.P. Bazinet, Brain elongation of linoleic acid is a negligible source of the arachidonate in brain phospholipids of adult rats, Biochim. Biophys. Acta - Mol. Cell Biol. Lipids. 1761 (2006) 1050-1059. doi:10.1016/j.bbalip.2006.06.006.

[27] J. Herz, H.H. Bock, Lipoprotein Receptors in the Nervous System, Annu. Rev. Biochem. 71 (2002) 405-434. doi:10.1146/annurev.biochem.71.110601.135342.

[28] J. Edmond, Essential polyunsaturated fatty acids and the barrier to the brain: The components of a model for transport, in: J. Mol. Neurosci., 2001: pp. 181-193. doi:10.1385/JMN:16:2-3:181.

[29] T. Rahman, A.Y. Taha, B. Jun Song, S.K. Orr, Z. Liu, C.T. Chen, R.P. Bazinet, The very low density lipoprotein receptor is not necessary for maintaining brain polyunsaturated fatty acid concentrations, Prostaglandins Leukot. Essent. Fat. Acids. 82 (2010) 141-145. doi:10.1016/j.plefa.2009.11.003.

[30] C.T. Chen, D.W.L. Ma, J.H. Kim, H.T.J. Mount, R.P. Bazinet, The low density lipoprotein receptor is not necessary for maintaining mouse brain polyunsaturated fatty acid concentrations, J. Lipid Res. 49 (2008) 147-152. doi:10.1194/jlr.M700386-JLR200.

[31] B.J. Song, A. Elbert, T. Rahman, S.K. Orr, C.T. Chen, M. Febbraio, R.P. Bazinet, Genetic ablation of CD36 does not alter mouse brain polyunsaturated fatty acid concentrations, Lipids. 45 (2010) 291-299. doi:10.1007/s11745-010-3398-z.

[32] M. Lagarde, N. Bernoud, N. Brossard, D. Lemaitre-Delaunay, F. Thiès, M. Croset, J. Lecerf, Lysophosphatidylcholine as a preferred carrier form of docosahexaenoic acid to the brain, J. Mol. Neurosci. 16 (2001) 201-204. doi:10.1385/JMN:16:2-3:201.

[33] N. Bernoud, L. Fenart, P. Molière, M.P. Dehouck, M. Lagarde, R. Cecchelli, J. Lecerf, Preferential transfer of 2-docosahexaenoyl-1-lysophosphatidylcholine through an in vitro blood-brain barrier over unesterified docosahexaenoic acid, J. Neurochem. 72 (1999) 338 345. doi:10.1046/j.1471-4159.1999.0720338.x.

[34] F. Thies, C. Pillon, P. Moliere, M. Lagarde, J. Lecerf, Preferential incorporation of sn-2 lysoPC DHA over unesterified DHA in the young rat brain., Am. J. Physiol. 267 (1994) R1273-9. doi:10.1152/ajpregu.1994.267.5.R1273.

[35] C.T. Chen, A.P. Kitson, K.E. Hopperton, A.F. Domenichiello, M.O. Trépanier, L.E. Lin, L. Ermini, M. Post, F. Thies, R.P. Bazinet, Plasma non-esterified docosahexaenoic acid is the major pool supplying the brain, Sci. Rep. 5 (2015). doi:10.1038/srep15791.

[36] M. Ouellet, V. Emond, C.T. Chen, C. Julien, F. Bourasset, S. Oddo, F. LaFerla, R.P. Bazinet, F. Calon, Diffusion of docosahexaenoic and eicosapentaenoic acids through the blood-brain barrier: An in situ cerebral perfusion study, Neurochem. Int. 55 (2009) 476482. doi:10.1016/j.neuint.2009.04.018. 
[37] W. Lefkowitz, S.Y. Lim, Y. Lin, N. Salem, Where does the developing brain obtain its docosahexaenoic acid? Relative contributions of dietary $\alpha$-linolenic acid, docosahexaenoic acid, and body stores in the developing rat, Pediatr. Res. 57 (2005) 157-165. doi:10.1203/01.PDR.0000147572.57627.AE.

[38] L. Liu, N. Bartke, H. Van Daele, P. Lawrence, X. Qin, H.G. Park, K. Kothapalli, A. Windust, J. Bindels, Z. Wang, J.T. Brenna, Higher efficacy of dietary DHA provided as a phospholipid than as a triglyceride for brain DHA accretion in neonatal piglets, J. Lipid Res. 55 (2014) 531-539. doi:10.1194/jlr.M045930.

[39] E. Sarkadi-Nagy, V. Wijendran, G.Y. Diau, A.C. Chao, A.T. Hsieh, A. Turpeinen, P. Lawrence, P.W. Nathanielsz, J.T. Brenna, Formula feeding potentiates docosahexaenoic and arachidonic acid biosynthesis in term and preterm baboon neonates, J. Lipid Res. 45 (2004) 71-80. doi:10.1194/jlr.M300106-JLR200.

[40] C.T. Chen, Z. Liu, R.P. Bazinet, Rapid de-esterification and loss of eicosapentaenoic acid from rat brain phospholipids: an intracerebroventricular study, J. Neurochem. 116 (2011) 363-373. doi:10.1111/j.1471-4159.2010.07116.x.

[41] Y.H. Lin, N. Salem, Whole body distribution of deuterated linoleic and alpha-linolenic acids and their metabolites in the rat., J. Lipid Res. 48 (2007) 2709-24. doi:10.1194/jlr.M700369-JLR200.

[42] W. Meier-Augenstein, H.F. Kemp, Stable Isotope Analysis: General Principles and Limitations, in: Wiley Encycl. Forensic Sci., John Wiley \& Sons, Ltd, Chichester, UK, 2012. doi:10.1002/9780470061589.fsa1041.

[43] M.H. O 'leary, S. Madhavan, P. Paneth, Physical and chemical basis of carbon isotope fractionation in plants, 15 (n.d.) 1099-1104.

[44] W. Meier-Augenstein, Stable isotope analysis of fatty acids by gas chromatographyisotope ratio mass spectrometry, Anal. Chim. Acta. 465 (2002) 63-79. doi:10.1016/S0003-2670(02)00194-0.

[45] J.. Carter, V.J. Barwick, Good Practice Guide for Isotope Ratio Mass Spectrometry, FIRMS. (2011) ISBN 978-0-948926-31-0.

[46] W.A. Brand, T.B. Coplen, Stable isotope deltas: tiny, yet robust signatures in nature, Isotopes Environ. Health Stud. 48 (2012) 393-409. doi:10.1080/10256016.2012.666977.

[47] A.A. Benson, C. M., The Path of Carbon in Photosynthesis, J. Exp. Bot. 1 (1950) 63-68. doi:10.1093/jxb/1.1.63.

[48] J.R. Ehleringer, Carbon Isotope Ratios and Physiological Processes in Aridland Plants, in: Springer, New York, NY, 1989: pp. 41-54. doi:10.1007/978-1-4612-3498-2_3.

[49] M.H. O 'leary. Carbon isotope fractionation in plants, Phytochemistry. 20 (1981) 5-567.

[50] R.F. Sage, M. Stata, Photosynthetic diversity meets biodiversity: The C4 plant example, J. 
Plant Physiol. 172 (2015) 104-119. doi:10.1016/J.JPLPH.2014.07.024.

[51] M.D. Hatch, C.R. Slack, Photosynthesis by sugar-cane leaves. A new carboxylation reaction and the pathway of sugar formation., Biochem. J. 101 (1966) 103-11.

[52] M.D. Hatch, C 4 photosynthesis: discovery and resolution, Photosynth. Res. 73 (2002) 251-256.

[53] M. O'Leary, Carbon isotopes in photosynthesis, Bioscience. 38 (1988) 328-336. doi: $10.2307 / 1310735$.

[54] N.J. van der Merwe, Carbon Isotopes, Photosynthesis, and Archaeology: Different pathways of photosynthesis cause characteristic changes in carbon isotope ratios that make possible the study of prehistoric human diets, Am. Sci. 70 (n.d.) 596-606. doi: $10.2307 / 27851731$.

[55] H. Griffiths, Carbon isotope discrimination and the integration of carbon assimilation pathways in terrestrial CAM plants, Plant, Cell Environ. 15 (1992) 1051-1062. doi:10.1111/j.1365-3040.1992.tb01655.x.

[56] W.G. Mook, J.C. Bommerson, W.H. Staverman, Carbon isotope fractionation between dissolved bicarbonate and gaseous cabon dioxide, Earth Planet. Sci. Lett. 22 (1974) 169176.

[57] B.N. Smith, S. Epsten, Two Categories of 13C/12C Ratios for Higher Plants, Plant PhysioL. 47 (1971) 380-384.

[58] R.L. France, Carbon-13 enrichment in benthic compared to planktonic algae: foodweb implications, Mar. Ecol. Prog. Ser. 124 (1995) 307-312. doi:10.3354/meps124307.

[59] D.E. Matthews, J.M. Hayes, Isotope-ratio-monitoring gas chromatography-mass spectrometry, Anal. Chem. 50 (1978) 1465-1473. doi:10.1021/ac50033a022.

[60] S.M. Budge, M.J. Wooller, A.M. Springer, S.J. Iverson, C.P. McRoy, G.J. Divoky, Tracing carbon flow in an arctic marine food web using fatty acid-stable isotope analysis, Oecologia. 157 (2008) 117-129. doi:10.1007/s00442-008-1053-7.

[61] M.M. Pollierer, J. Dyckmans, S. Scheu, D. Haubert, J. Dyckmans2, S. Scheu1, D. Haubert3, Carbon flux through fungi and bacteria into the forest soil animal food web as indicated by compound-specific $13 \mathrm{C}$ fatty acid analysis Carbon flux through fungi and bacteria into soil animal food web as indicated by compound specific $13 \mathrm{C}$ fatty acid analysis, Source Funct. Ecol. Funct. Ecol. 26 (2012) 978-990. doi:10.1111/j.

[62] R.P. Evershed, K.I. Arnot, J. Collister, G. Eglinton, S. Charters, Application of isotope ratio monitoring gas chromatography-mass spectrometry to the analysis of organic residues of archaeological origin, Analyst. 119 (1994) 909-914. doi:10.1039/AN9941900909.

[63] Dudd, Evershed, Direct demonstration of milk as an element of archaeological economies, 
Science. 282 (1998) 1478-81.

[64] S.E. Woodbury, R.P. Evershed, J.B. Rossell, R.E. Griffith, P. Farnell, Detection of Vegetable Oil Adulteration Using Gas Chromatography Combustion/Isotope Ratio Mass Spectrometry, Anal. Chem. 67 (1995) 2685-2690. doi:10.1021/ac00111a029.

[65] F. Angerosa, L. Camera, S. Cumitini, A. G. Gleixner, F. Reniero, Carbon Stable Isotopes and Olive Oil Adulteration with Pomace Oil, (1997). doi:10.1021/JF960993D.

[66] H. Demmelmair, U. v. Schenck, E. Behrendt, T. Sauerwald, B. Koletzko, Estimation of arachidonic acid synthesis in full term neonates using natural variation of $13 \mathrm{C}$ content, $\mathrm{J}$ Pediatr Gastroenterol Nutr. 21 (1995) 21-36. doi:10.1097/00005176-199507000-00005.

[67] V.P. Carnielli, M. Simonato, G. Verlato, I. Luijendijk, M. De Curtis, P.J. Sauer, P.E. Cogo, Synthesis of long-chain polyunsaturated fatty acids in preterm newborns fed formula with long-chain polyunsaturated fatty acids., Am. J. Clin. Nutr. 86 (2007) 132330 .

[68] S.K. Rhee, R.G. Reed, J.T. Brenna, Fatty Acid Carbon Isotope Ratios in Humans on Controlled Diets, 32 (1997).

[69] A.H. Metherel, R. Chouinard-Watkins, M.-O. Trépanier, R.J.S. Lacombe, R.P. Bazinet, Retroconversion is a minor contributor to increases in eicosapentaenoic acid following docosahexaenoic acid feeding as determined by compound specific isotope analysis in rat liver., Nutr. Metab. (Lond). 14 (2017) 75. doi:10.1186/s12986-017-0230-2.

[70] R.J.S. Lacombe, V. Giuliano, S.M. Colombo, M.T. Arts, R.P. Bazinet, Compoundspecific isotope analysis resolves the dietary origin of docosahexaenoic acid in the mouse brain, J. Lipid Res. 58 (2017) 2071-2081. doi:10.1194/j1r.D077990.

[71] J.X. Kang, J. Wang, L. Wu, Z.B. Kang, Transgenic mice: fat-1 mice convert n-6 to n-3 fatty acids., Nature. 427 (2004) 504. doi:10.1038/427504a.

[72] Y. Ge, X. Wang, Z. Chen, N. Landman, E.H. Lo, J.X. Kang, Gene transfer of the Caenorhabditis elegans n-3 fatty acid desaturase inhibits neuronal apoptosis., J. Neurochem. 82 (2002) 1360-6.

[73] Z.B. Kang, Y. Ge, Z. Chen, J. Cluette-Brown, M. Laposata, A. Leaf, J.X. Kang, Adenoviral gene transfer of Caenorhabditis elegans n--3 fatty acid desaturase optimizes fatty acid composition in mammalian cells., Proc. Natl. Acad. Sci. U. S. A. 98 (2001) 4050-4. doi:10.1073/pnas.061040198.

[74] Y. Ge, Z. Chen, Z.B. Kang, J. Cluette-Brown, M. Laposata, J.X. Kang, Effects of adenoviral gene transfer of C. elegans n-3 fatty acid desaturase on the lipid profile and growth of human breast cancer cells., Anticancer Res. 22 (2002) 537-43.

[75] S. Xia, Y. Lu, J. Wang, C. He, S. Hong, C.N. Serhan, J.X. Kang, Melanoma growth is reduced in fat- 1 transgenic mice: impact of omega-6/omega-3 essential fatty acids., Proc. Natl. Acad. Sci. U. S. A. 103 (2006) 12499-504. doi:10.1073/pnas.0605394103. 
[76] J.A. Napier, L. V. Michaelson, Genomic and functional characterization of polyunsaturated fatty acid biosynthesis in caenorhabditis elegans, Lipids. 36 (2001) 761766. doi:10.1007/s11745-001-0782-9.

[77] D. Meesapyodsuk, D.W. Reed, C.K. Savile, P.H. Buist, S.J. Ambrose, P.S. Covello, Characterization of the regiochemistry and cryptoregiochemistry of a Caenorhabditis elegans fatty acid desaturase (FAT-1) expressed in Saccharomyces cerevisiae., Biochemistry. 39 (2000) 11948-54.

[78] M. Okabe, M. Ikawa, K. Kominami, T. Nakanishi, Y. Nishimune, "Green mice" as a source of ubiquitous green cells., FEBS Lett. 407 (1997) 313-9.

[79] Z. Gao, Y. Wang, Z. Ren, Q. Li, Y. Wang, Y. Dai, Genome-wide screen for serum microRNA expression profile in mfat-1 transgenic mice, Tumor Biol. 35 (2014) 9717 9723. doi:10.1007/s13277-014-2261-x.

[80] G. Astarita, J.H. McKenzie, B. Wang, K. Strassburg, A. Doneanu, J. Johnson, A. Baker, T. Hankemeier, J. Murphy, R.J. Vreeken, J. Langridge, J.X. Kang, A protective lipidomic biosignature associated with a balanced omega-6/omega-3 ratio in fat-1 transgenic mice, PLoS One. 9 (2014). doi:10.1371/journal.pone.0096221.

[81] K.E. Hopperton, M.-O. Trépanier, V. Giuliano, R.P. Bazinet, Brain omega-3 polyunsaturated fatty acids modulate microglia cell number and morphology in response to intracerebroventricular amyloid- $\beta$ 1-40 in mice, J. Neuroinflammation. 13 (2016) 257. doi:10.1186/s12974-016-0721-5.

[82] S.K. Orr, S. Palumbo, F. Bosetti, H.T. Mount, J.X. Kang, C.E. Greenwood, D.W.L. Ma, C.N. Serhan, R.P. Bazinet, R.P. Bazinet, Unesterified docosahexaenoic acid is protective in neuroinflammation., J. Neurochem. 127 (2013) 378-93. doi:10.1111/jnc.12392.

[83] C. He, X. Qu, L. Cui, J. Wang, J.X. Kang, Improved spatial learning performance of fat-1 mice is associated with enhanced neurogenesis and neuritogenesis by docosahexaenoic acid., Proc. Natl. Acad. Sci. U. S. A. 106 (2009) 11370-5. doi:10.1073/pnas.0904835106.

[84] S.-N. Lim, S.J. Gladman, S.C. Dyall, U. Patel, N. Virani, J.X. Kang, J. V. Priestley, A.T. Michael-Titus, Transgenic mice with high endogenous omega-3 fatty acids are protected from spinal cord injury, Neurobiol. Dis. 51 (2013) 104-112. doi:10.1016/j.nbd.2012.10.021.

[85] C.A. Hudert, K.H. Weylandt, Y. Lu, J. Wang, S. Hong, A. Dignass, C.N. Serhan, J.X. Kang, Transgenic mice rich in endogenous omega-3 fatty acids are protected from colitis., Proc. Natl. Acad. Sci. U. S. A. 103 (2006) 11276-81. doi:10.1073/pnas.0601280103.

[86] M.M. Rahman, A. Bhattacharya, J. Banu, J.X. Kang, G. Fernandes, Endogenous n-3 fatty acids protect ovariectomy induced bone loss by attenuating osteoclastogenesis., J. Cell. Mol. Med. 13 (2009) 1833-44. doi:10.1111/j.1582-4934.2009.00649.x.

[87] J.-B. Wan, L.-L. Huang, R. Rong, R. Tan, J. Wang, J.X. Kang, Endogenously Decreasing Tissue n-6/n-3 Fatty Acid Ratio Reduces Atherosclerotic Lesions in Apolipoprotein E- 
Deficient Mice by Inhibiting Systemic and Vascular Inflammation, Arterioscler. Thromb. Vasc. Biol. 30 (2010) 2487-2494. doi:10.1161/ATVBAHA.110.210054.

[88] S.K. Orr, J.Y.M. Tong, J.X. Kang, D.W.L. Ma, R.P. Bazinet, The Fat-1 mouse has brain docosahexaenoic acid levels achievable through fish oil feeding, Neurochem. Res. 35 (2010) 811-819. doi:10.1007/s11064-010-0139-x.

[89] J.T. Brenna, G.-Y. Diau, The influence of dietary docosahexaenoic acid and arachidonic acid on central nervous system polyunsaturated fatty acid composition, Prostaglandins, Leukot. Essent. Fat. Acids. 77 (2007) 247-250. doi:10.1016/j.plefa.2007.10.016.

[90] M. Martínez, I. Mougan, Fatty acid composition of human brain phospholipids during normal development., J. Neurochem. 71 (1998) 2528-2533. doi:10.1046/j.14714159.1998.71062528.x.

[91] M.A. Beydoun, M.T. Fanelli Kuczmarski, H.A. Beydoun, J.R. Hibbeln, M.K. Evans, A.B. Zonderman, $\omega-3$ Fatty Acid Intakes Are Inversely Related to Elevated Depressive Symptoms among United States Women., J. Nutr. 143 (2013) 1743-1752. doi:10.3945/jn.113.179119.

[92] S. Layé, A. Nadjar, C. Joffre, R.P. Bazinet, Anti-Inflammatory Effects of Omega-3 Fatty Acids in the Brain: Physiological Mechanisms and Relevance to Pharmacology., Pharmacol. Rev. 70 (2018) 12-38. doi:10.1124/pr.117.014092.

[93] R.J.S. Lacombe, R. Chouinard-Watkins, R.P. Bazinet, Brain docosahexaenoic acid uptake and metabolism, Mol. Aspects Med. (2018). doi:10.1016/j.mam.2017.12.004.

[94] Z. Muccio, G.P. Jackson, Isotope Ratio Mass Spectrometry., Analyst. 134 (2009) 213-22. doi:10.1039/b808232d.

[95] J.T. Brenna, T.N. Corso, H.J. Tobias, R.J. Caimi, High-precision continuous-flow isotope ratio mass spectrometry, Mass Spectrom. Rev. 16 (1997) 227-258. doi:10.1002/(SICI)1098-2787(1997)16:5<227::AID-MAS1>3.0.CO;2-J.

[96] G.D. Farquhar, J.R. Ehleringer, K.T. Hubick, Carbon Isotope Discrimination and Photosynthesis, Annu. Rev. Plant Physiol. Plant Mol. Biol. 40 (1989) 503-537. doi:10.1146/annurev.pp.40.060189.002443.

[97] Gannes L.Z., Martinez del Rio C., Koch P., Natural Abundance Variations in Stable Isotopes and their Potential Uses in Animal Physiological Ecology, Comp. Biochem. Physiol. 119A (1998) 725-737.

[98] J.X. Kang, Fat-1 transgenic mice: A new model for omega-3 research, Prostaglandins Leukot. Essent. Fat. Acids. 77 (2007) 263-267. doi:10.1016/j.plefa.2007.10.010.

[99] J. Folch, M. Lees, G.H. Sloane Stanley, A simple method for the isolation and purification of total lipides from animal tissues., J. Biol. Chem. 226 (1957) 497-509.

[100] W.. Morrison, L.M. Smith, Preparation of Fatty Acid Methyl Esters and Dimethyl Lipids 
from Lipids with Boron Fluoride-Methanol, J. Lipid Res. 5 (1964) 600-8.

[101] M. Igarashi, K. Ma, L. Chang, J.M. Bell, S.I. Rapoport, J.C. DeMar Jr., Low liver conversion rate of alpha-linolenic to docosahexaenoic acid in awake rats on a highdocosahexaenoate-containing diet, J Lipid Res. 47 (2006) 1812-1822. doi:M600030JLR200 [pii]ไr10.1194/jlr.M600030-JLR200.

[102] H.P. Cho, M.T. Nakamura, S.D. Clarke, Cloning, expression, and nutritional regulation of the mammalian Delta-6 desaturase., J. Biol. Chem. 274 (1999) 471-7. doi:10.1074/JBC.274.1.471.

[103] H.P. Cho, M. Nakamura, S.D. Clarke, Cloning, expression, and fatty acid regulation of the human delta-5 desaturase., J. Biol. Chem. 274 (1999) 37335-9. doi:10.1074/JBC.274.52.37335.

[104] P.T. Price, C.M. Nelson, S.D. Clarke, Omega-3 polyunsaturated fatty acid regulation of gene expression, Curr Opin Lipidol. 11 (2000) 3-7. doi:10.1097/00041433-20000200000002 .

[105] J.C. DeMar, K. Ma, J.M. Bell, S.I. Rapoport, Half-lives of docosahexaenoic acid in rat brain phospholipids are prolonged by 15 weeks of nutritional deprivation of $n-3$ polyunsaturated fatty acids, J. Neurochem. 91 (2004) 1125-1137. doi:10.1111/j.14714159.2004.02789.x.

[106] N. Wolf, S.A. Carleton, C. Martínez, D. Rio, Ten years of experimental animal isotopic ecology, Funct. Ecol. 23 (2009) 17-26. doi:10.1111/j.1365-2435.2008.01529.x.

[107] S.M. Hixson, C.C. Parrish, D.M. Anderson, Changes in Tissue Lipid and Fatty Acid Composition of Farmed Rainbow Trout in Response to Dietary Camelina Oil as a Replacement of Fish Oil, Lipids. 49 (2014) 97-111. doi:10.1007/s11745-013-3862-7.

[108] K. Choy, S.H. Nash, A.R. Kristal, S. Hopkins, B.B. Boyer, D.M. O’Brien, The carbon isotope ratio of alanine in red blood cells is a new candidate biomarker of sugar-sweetened beverage intake., J. Nutr. 143 (2013) 878-84. doi:10.3945/jn.112.172999. 


\section{Chapter 6:}

\section{Appendix 1}

Confirmation of dietary fatty acid source via natural abundance compound specific isotope analysis 


\subsection{Isotopic analysis of dietary fatty acids}

In the present analysis, we utilized CSIA by IRMS to confirm the fatty acid source of our purchased diets (Table 6.1). The main n-6 PUFA (LA) in the CO and CO FO diets were derived from corn oil, a C4 plant source. As a result, LA within these diets should reflect a higher enrichment in ${ }^{13} \mathrm{C}$ than fatty acids derived from $\mathrm{C} 3$ plant sources such as $\mathrm{SO}$ [57]. Although LA isotopically analyzed from the COFO diet did reflect a greater ${ }^{13} \mathrm{C}$ enrichment than LA derived from $\mathrm{SO}$, this was not the case for LA analyzed in the initially purchased CO diet. LA within this diet displayed a mean isotopic signature of $-32.32 \mathrm{mUr}$, far too deplete to be derived from a $\mathrm{CO}$ source. This data made it clear that an error had been made in the synthesis of our purchased $\mathrm{CO}$ diet. This was further confirmed by subsequent fatty acid analysis by GC-FID that revealed a fatty acid composition uncharacteristic of $\mathrm{CO}$ (data not shown). As a result, this diet was not utilized and a new $\mathrm{CO}$ diet was purchased (CO diet repurchased). Upon isotopic analysis, LA derived from this diet displayed $\mathrm{LA} \delta^{13} \mathrm{C}$ values within the reported range of a $\mathrm{C} 4$ plant.

Fatty acid isotopic analysis from other diets were consistent with their expected source. In the CO FO and SOFO diets, the main n-3 PUFA (DHA and EPA) were solely derived from a marine fish source, menhaden. This was confirmed in our isotopic analysis, as the $\delta^{13} \mathrm{C}$ values of these fatty acids were within the reported range for marine fish sources[57]. Further, the main n6 PUFA present within the SO and SOFO diet (LA) was derived from a C3 plant source, safflower. The relatively ${ }^{13} \mathrm{C}$ deplete $\delta^{13} \mathrm{C}$ values of dietary LA obtained by CSIA by IRMS reflected this.

All in all, this analysis reveals the valuable use of CSIA by IRMS to confirm the dietary fatty acid source of experimental diets. 


\begin{tabular}{ccc}
\hline Diet & Fatty acid & $\delta^{13} \mathrm{C}(\mathrm{mUr})$ \\
\hline $10 \%$ Corn oil (initially purchased) & LA & -32.32 \\
$10 \%$ Corn oil (repurchased) & LA & -16.51 \\
$8 \%$ Corn oil, $2 \%$ fish oil & LA & -16.15 \\
& DHA & -21.98 \\
$10 \%$ Safflower oil & EPA & -24.28 \\
& LA & -31.86 \\
& LA & -30.67 \\
& DHA & -21.99 \\
\hline
\end{tabular}

Table 6.1: $\delta^{13} \mathrm{C}$ values of key fatty acids in experimental diets 


\section{Chapter 7:}

Appendix 2

Whole brain fatty acid composition 


\begin{tabular}{|c|c|c|c|c|c|c|c|}
\hline \multirow[b]{2}{*}{ Name } & \multicolumn{2}{|c|}{ Wild-type } & \multicolumn{2}{|r|}{ Fat-1 } & \multirow{2}{*}{$\begin{array}{c}\text { Interaction } \\
\text { effect }\end{array}$} & \multirow[t]{2}{*}{ Diet effect } & \multirow[t]{2}{*}{ Genotype effect } \\
\hline & $10 \%$ Safflower & $\begin{array}{c}8 \% \text { Safflower, } \\
2 \% \text { fish oil }\end{array}$ & $10 \%$ Safflower & $\begin{array}{l}8 \% \text { Safflower, } 2 \% \text { fish } \\
\text { oil }\end{array}$ & & & \\
\hline C $14: 0$ & $473 \pm 234$ & $425 \pm 45$ & $398 \pm 36$ & $429 \pm 27$ & & & \\
\hline C $16: 0$ & $43047 \pm 5929$ & $44350 \pm 2940$ & $44293 \pm 2296$ & $46002 \pm 1447$ & & & \\
\hline C 18:0 & $33268 \pm 3199$ & $35571 \pm 2318$ & $35620 \pm 1569$ & $36724 \pm 1239$ & & & \\
\hline C 20:0 & $628 \pm 71$ & $668 \pm 66$ & $627 \pm 45$ & $659 \pm 34$ & & & \\
\hline C 22:0 & $420 \pm 52$ & $477 \pm 50$ & $435 \pm 27$ & $472 \pm 24$ & & $* *$ & \\
\hline C 23:0 & $146 \pm 27$ & $174 \pm 16$ & $159 \pm 7$ & $168 \pm 7$ & & $*$ & \\
\hline C $24: 0$ & $26 \pm 26$ & $86 \pm 5$ & $76 \pm 6$ & $166 \pm 34$ & & $* * *$ & $* * *$ \\
\hline \multicolumn{8}{|l|}{ SFAs } \\
\hline C $14: 1$ & $3844 \pm 398^{\mathrm{a}}$ & $4626 \pm 414^{b}$ & $4693 \pm 514^{b}$ & $4650 \pm 209^{b}$ & $*$ & & \\
\hline C $16: 1$ & $985 \pm 178^{a}$ & $1103 \pm 80^{\mathrm{a}}$ & $1012 \pm 46^{\mathrm{a}}$ & $1134 \pm 57^{\mathrm{b}}$ & $*$ & & \\
\hline C $18: 1 \mathrm{n}-7$ & $6685 \pm 646$ & $6614 \pm 503$ & $6551 \pm 247$ & $6735 \pm 181$ & & & \\
\hline C $18: 1 n-9$ & $26305 \pm 2748$ & $30775 \pm 2377$ & $30010 \pm 1246$ & $32165 \pm 895$ & & $* * *$ & $* *$ \\
\hline C $20: 1 \mathrm{n}-9$ & $2512 \pm 446$ & $3085 \pm 321$ & $2976 \pm 228$ & $3170 \pm 148$ & & $* *$ & $*$ \\
\hline C $24: 1 \mathrm{n}-9$ & $1015 \pm 168$ & $1216 \pm 135$ & $1073 \pm 88$ & $1191 \pm 61$ & & $* *$ & \\
\hline \multicolumn{8}{|l|}{ MUFAs } \\
\hline C $18: 2 n-6$ & $1590 \pm 234$ & $1493 \pm 129$ & $1712 \pm 113$ & $1770 \pm 164$ & & & $* *$ \\
\hline C $20: 2 n-6$ & $516 \pm 66^{\mathrm{a}}$ & $392 \pm 29^{b}$ & $464 \pm 18^{\mathrm{a}}$ & $427 \pm 23^{\mathbf{b}}$ & $*$ & & \\
\hline C $20: 3 n-6$ & $512 \pm 112$ & $770 \pm 54$ & $695 \pm 19$ & $928 \pm 59$ & & $* * *$ & $* * *$ \\
\hline C $22: 4 n-6$ & $3982 \pm 205^{a}$ & $2724 \pm 238^{b}$ & $3088 \pm 137^{\mathrm{c}}$ & $2516 \pm 153^{b}$ & $* * *$ & & \\
\hline C $22: 5 n-6$ & $8732 \pm 1101^{\mathrm{a}}$ & $913 \pm 39^{\mathrm{b}}$ & $560 \pm 48^{\mathrm{b}}$ & $385 \pm 229^{b}$ & $* * *$ & & \\
\hline \multicolumn{8}{|l|}{ N-6 } \\
\hline C $18: 3 n-3$ & $781 \pm 92$ & $849 \pm 88$ & $796 \pm 53$ & $855 \pm 47$ & & $*$ & \\
\hline C $20: 5 n-3$ & $622 \pm 92$ & $712 \pm 91$ & $649 \pm 67$ & $719 \pm 51$ & & $*$ & \\
\hline C $22: 5 n-3$ & $64 \pm 24$ & $244 \pm 114$ & $129 \pm 100$ & $323 \pm 138$ & & $* * *$ & \\
\hline $\mathrm{N}-3$ & & & & & & & \\
\hline
\end{tabular}

Table 7.1 Brain whole brain fatty acid concentrations ( $\mathrm{nmol} / \mathrm{g}$ ) of wild-type and fat-1 mice fed the $10 \%$ safflower oil and $8 \%$ safflower oil, $2 \%$ fish oil diet

Values represent whole brain fatty acid concentrations (nmol/g) of wild-type and fat- 1 mice measured by GC-FID. Upon weaning, mice either remained on the SO diet or were switched onto the SOFO diet. They remained on this diet until euthanasia at 12 weeks of age. Data is presented as mean $\pm \mathrm{SD}$ ( $\mathrm{n}=6-7$ per group). Data was statistically analyzed via two-way ANOVA and Tukey's HSD test was utilized for post hoc analysis when a significant interaction effect was present. Columns with asterisks represent statistical. Blank columns indicate that the measure was not statistically significant. ${ }^{*}=\mathrm{p}<0.05,{ }^{* *}=\mathrm{p}<0.01,{ }^{* * *}=\mathrm{p}<0.001$. MUFA monounsaturated fatty acids, SFA saturated fatty acids, $N-6$ omega-6, $N-3$ omega 


\begin{tabular}{|c|c|c|c|c|c|c|c|}
\hline \multirow[b]{2}{*}{ Name } & \multicolumn{2}{|c|}{ Wild-type } & \multicolumn{2}{|c|}{ Fat-1 } & \multirow[t]{2}{*}{ Interaction effect } & \multirow[t]{2}{*}{ Diet effect } & \multirow[t]{2}{*}{ Genotype effect } \\
\hline & $10 \%$ Corn oil & $\begin{array}{c}8 \% \text { Corn oil, } \\
2 \% \text { fish oil }\end{array}$ & $10 \%$ Corn & $\begin{array}{c}8 \% \text { Corn oil, } \\
2 \% \text { fish oil }\end{array}$ & & & \\
\hline C $14: 0$ & $312 \pm 20$ & $310 \pm 16$ & $306 \pm 44$ & $318 \pm 29$ & & & \\
\hline C $16: 0$ & $37539 \pm 1328$ & $36321 \pm 2455$ & $36883 \pm 5121$ & $36268 \pm 3201$ & & & \\
\hline C $18: 0$ & $31665 \pm 531$ & $30994 \pm 2012$ & $31094 \pm 3766$ & $30587 \pm 2229$ & & & \\
\hline C 20:0 & $607 \pm 20$ & $570 \pm 38$ & $603 \pm 85$ & $587 \pm 65$ & & & \\
\hline C 22:0 & $452 \pm 24$ & $436 \pm 42$ & $466 \pm 52$ & $449 \pm 55$ & & & \\
\hline C 23:0 & $154 \pm 13$ & $150 \pm 14$ & $156 \pm 13$ & $152 \pm 16$ & & & \\
\hline C $24: 0$ & $601 \pm 42$ & $587 \pm 69$ & $629 \pm 66$ & $601 \pm 96$ & & & \\
\hline \multicolumn{8}{|l|}{ SFAs } \\
\hline C $14: 1$ & $2536 \pm 137$ & $2588 \pm 242$ & $2613 \pm 407$ & $2433 \pm 435$ & & & \\
\hline C $16: 1$ & $837 \pm 62$ & $867 \pm 44$ & $854 \pm 125$ & $885 \pm 75$ & & & \\
\hline C $18: 1 n-7$ & $7443 \pm 1315$ & $6264 \pm 725$ & $6923 \pm 1867$ & $6825 \pm 1266$ & & & \\
\hline C $18: \ln -9$ & $26964 \pm 1678$ & $27399 \pm 1150$ & $28646 \pm 4285$ & $28879 \pm 2239$ & & & \\
\hline C 20:1n-9 & $2698 \pm 140$ & $2685 \pm 180$ & $2941 \pm 411$ & $2823 \pm 311$ & & & \\
\hline C $24: 1 n-9$ & $1164 \pm 150$ & $1067 \pm 56$ & $1241 \pm 151$ & $1152 \pm 213$ & & & \\
\hline \multicolumn{8}{|l|}{ MUFAs } \\
\hline C $18: 2 n-6$ & $1242 \pm 78$ & $1226 \pm 113$ & $1437 \pm 169$ & $1355 \pm 97$ & & & $*$ \\
\hline C $20: 2 n-6$ & $419 \pm 21$ & $332 \pm 34$ & $428 \pm 33$ & $363 \pm 37$ & & $* * *$ & \\
\hline C $20: 3 n-6$ & $460 \pm 19$ & $709 \pm 50$ & $650 \pm 84$ & $860 \pm 49$ & & $* * *$ & $* * *$ \\
\hline C $20: 4 n-6$ & $13905 \pm 569$ & $12249 \pm 771$ & $13049 \pm 1598$ & $11415 \pm 690$ & & $* *$ & \\
\hline C $22: 4 n-6$ & $3386 \pm 200$ & $2491 \pm 178$ & $2779 \pm 291$ & $1954 \pm 835$ & & $* *$ & * \\
\hline C $22: 5 n-6$ & $3612 \pm 605^{\mathrm{a}}$ & $914 \pm 69^{b}$ & $428 \pm 35^{\mathrm{b}}$ & $306 \pm 12^{\mathrm{b}}$ & $* * *$ & & \\
\hline \multicolumn{8}{|l|}{ N-6 } \\
\hline C $18: 3 n-3$ & $757 \pm 444$ & $732 \pm 50$ & $772 \pm 127$ & $758 \pm 90$ & & & \\
\hline C $20: 5 n-3$ & $40 \pm 27^{\mathrm{a}}$ & $92 \pm 46^{b}$ & $92 \pm 19^{b}$ & $230 \pm 22^{c}$ & $* *$ & & \\
\hline C $22: 5 n-3$ & $757 \pm 43$ & $318 \pm 19$ & $772 \pm 126$ & $455 \pm 34$ & & $* * *$ & * \\
\hline
\end{tabular}

Table 7.2 Brain whole brain fatty acid concentrations (nmol/g) of wild-type and fat-1 mice fed the $10 \%$ corn oil and $8 \%$ corn oil, $2 \%$ fish oil diet

Values represent whole brain fatty acid concentrations (nmol/g) of wild-type and fat-1 mice measured by GC-FID. Upon weaning, mice either remained on the CO diet or were switched onto the COFO diet. They remained on this diet until euthanasia at 12 weeks of age. Data is presented as mean $\pm \mathrm{SD}(\mathrm{n}=4-6$ per group). Data was statistically analyzed via two-way ANOVA and Tukey's HSD test was utilized for post hoc analysis when a significant interaction effect was present. Columns with asterisks represent statistical. Blank columns indicate that the measure was not statistically significant. $*=\mathrm{p}<0.05, * *=\mathrm{p}<0.01, * * *=\mathrm{p}<0.001$. MUFA monounsaturated fatty acids, SFA saturated fatty acids, $N-6$ omega-6, $N-3$ omega-3. 